\title{
HAMILTONIAN 2-FORMS IN KÄHLER GEOMETRY, III EXTREMAL METRICS AND STABILITY
}

\author{
VESTISLAV APOSTOLOV, DAVID M. J. CALDERBANK, PAUL GAUDUCHON, \\ AND CHRISTINA W. TØNNESEN-FRIEDMAN
}

\begin{abstract}
This paper concerns the explicit construction of extremal Kähler metrics on total spaces of projective bundles, which have been studied in many places. We present a unified approach, motivated by the theory of hamiltonian 2 -forms (as introduced and studied in previous papers in the series) but this paper is largely independent of that theory.

We obtain a characterization, on a large family of projective bundles, of those 'admissible' Kähler classes (i.e., the ones compatible with the bundle structure in a way we make precise) which contain an extremal Kähler metric. In many cases, such as on geometrically ruled surfaces, every Kähler class is admissible. In particular, our results complete the classification of extremal Kähler metrics on geometrically ruled surfaces, answering several long-standing questions.

We also find that our characterization agrees with a notion of K-stability for admissible Kähler classes. Our examples and nonexistence results therefore provide a fertile testing ground for the rapidly developing theory of stability for projective varieties, and we discuss some of the ramifications. In particular we obtain examples of projective varieties which are destabilized by a non-algebraic degeneration.
\end{abstract}

In this paper we give a systematic overview of some explicit constructions of extremal Kähler metrics on projective bundles and relate our constructions to the theory of stability for algebraic varieties. Recall that a Kähler metric $(g, J, \omega)$ on a manifold $M$ is said to be extremal [7] if the scalar curvature is a Killing potential, i.e., its symplectic gradient is a Killing vector field, or equivalently, its gradient is a holomorphic vector field. If $(M, J)$ is compact, the extremal Kähler metrics are exactly the critical points of the $L_{2}$-norm of the scalar curvature on the space of compatible Kähler metrics in a fixed Kähler class $\Omega=[\omega]$.

There has been a great deal of interest recently in the relation between extremal Kähler metrics, especially constant scalar curvature (CSC) Kähler metrics, and stability: roughly speaking there are conjectures that the existence of an extremal or CSC Kähler metric in an integral Kähler class $\Omega$ on a compact complex manifold $M$ should be equivalent to an algebraic geometric stability condition on the Kodaira embeddings of $M$ into $P\left(H^{0}\left(M, L^{k}\right)^{*}\right)$ for $k \gg 1$ where $c_{1}(L)=\Omega / 2 \pi$.

Our own interest in constructions of extremal Kähler metrics has been renewed and stimulated not only by these developments, but also by a unifying principle, which we shall explain and apply here, underlying explicit examples of such metrics on projective bundles obtained in [8, 22, 25, 26, 46. In short these examples have in common the presence of a hamiltonian 2-form of order 1 .

A hamiltonian 2-form [4] on a Kähler manifold $(M, J, g, \omega)$, of real dimension $2 m>2$ is a real $(1,1)$-form (i.e., a $J$-invariant 2 -form) $\phi$ such that

$$
2 \nabla_{X} \phi=d \operatorname{tr} \phi \wedge(J X)^{b}-(J d \operatorname{tr} \phi) \wedge X^{b}
$$

Date: October 16, 2018.

The first author was supported by NSERC grant OGP0023879, the second author by an EPSRC Advanced Research Fellowship and the fourth author by the Union College Faculty Research Fund. 
for all $X \in T M$ (where $X^{b}(Y)=g(X, Y)$ for $Y \in T M$ and $\operatorname{tr} \phi=\langle\omega, \phi\rangle_{g}$ ). The momentum polynomial of $\phi$ is then defined to be

$$
p(t):=(-1)^{m} \operatorname{pf}(\phi-t \omega)=t^{m}-(\operatorname{tr} \phi) t^{m-1}+\cdots+(-1)^{m} \operatorname{pf} \phi,
$$

where the pfaffian is defined by $\phi \wedge \cdots \wedge \phi=(\operatorname{pf} \phi) \omega \wedge \cdots \wedge \omega$. The reason $\phi$ is called hamiltonian is that for any $t \in \mathbb{R}, p(t)$ is a hamiltonian for a Killing vector field $K(t):=J \operatorname{grad}_{g} p(t)$ (this is not difficult to show [4, §2]). The integer $\ell=\max _{x \in M} \operatorname{dim} \operatorname{span}\left\{K(t)_{x}: t \in \mathbb{R}\right\}$ (with $0 \leq \ell \leq m$ ) is called the order of $\phi$.

We do not wish to impose the study of hamiltonian 2-forms on the reader of this paper, since we only need the general theory as motivation for the classes of complex manifolds and metrics that we shall study. We therefore now state a classification result which reduces the theory of hamiltonian 2-forms of order 1 to an Ansatz for metrics on projective bundles. This result follows easily from [4, 5, as we explain in Appendix A, where we also explain why we restrict attention to the order 1 case.

Theorem 1. Let $(M, g, J, \omega)$ be a compact connected Kähler $2 m$-manifold with a hamiltonian 2-form $\phi$ of order 1 . Then:

- there is an effective isometric hamiltonian $S^{1}$ action on $M$ generated by a vector field $K=J \operatorname{grad}_{g} z$ such that the stable quotient of $M$ by the induced holomorphic $\mathbb{C}^{\times}$action is a compact connected complex manifold $\hat{S}$ of real dimension $2(m-1)$;

- without loss, the image of the momentum map $z$ is $[-1,1]$, and there are Kähler manifolds $S_{a}$ of dimension $2 d_{a}$ and real numbers $x_{a}$, indexed by $a \in \hat{\mathcal{A}} \subset \mathbb{N} \cup\{\infty\}$, such that $\hat{S}$ is covered by $\prod_{a \in \hat{\mathcal{A}}} S_{a}$ and $0<\left|x_{a}\right| \leq 1$ with equality iff $a \in\{0, \infty\}$;

- $z$ is a Morse-Bott function [1] on $M$ with critical set $z^{-1}(\{-1,1\}), M^{0}:=$ $z^{-1}((-1,1))$ is a principal $\mathbb{C}^{\times}$-bundle over $\hat{S}$, and on each $S_{a}$ there is a Kähler metric $\left( \pm g_{a}, \pm \omega_{a}\right)$, with the sign chosen so that $\omega_{a} / x_{a}$ is positive, such that the Kähler structure on $M^{0}$ is

$$
g=\sum_{a \in \hat{\mathcal{A}}} \frac{1+x_{a} z}{x_{a}} g_{a}+\frac{d z^{2}}{\Theta(z)}+\Theta(z) \theta^{2}, \quad \omega=\sum_{a \in \hat{\mathcal{A}}} \frac{1+x_{a} z}{x_{a}} \omega_{a}+d z \wedge \theta,
$$

where $\theta$ is a connection 1 -form $(\theta(K)=1)$ with $d \theta=\sum_{a \in \hat{\mathcal{A}}} \omega_{a}$, and $\Theta$ is a smooth function on $[-1,1]$ satisfying

$$
\begin{aligned}
\Theta & >0 \quad \text { on } \quad(-1,1), \\
\Theta( \pm 1)=0, & \Theta^{\prime}( \pm 1)=\mp 2 ;
\end{aligned}
$$

- if $0 \in \hat{\mathcal{A}}$ then $x_{0}=1, S_{0}=\mathbb{C} P^{d_{0}}$ and $g_{0}$ is the Fubini-Study metric of scalar curvature $2 d_{0}\left(d_{0}+1\right)$, otherwise we set $d_{0}=0$; likewise, if $\infty \in \hat{\mathcal{A}}$ then $x_{\infty}=-1$, $S_{\infty}=\mathbb{C} P^{d_{\infty}}$ and $-g_{\infty}$ is the Fubini-Study metric of scalar curvature $2 d_{\infty}\left(d_{\infty}+1\right)$, otherwise we set $d_{\infty}=0$; we also put $\mathcal{A}=\hat{\mathcal{A}} \backslash\{0, \infty\} \subset \mathbb{Z}^{+}$;

- the blow-up $\hat{M}$ of $M$ along $z^{-1}(\{-1,1\})$ is $\mathbb{C}^{\times}$-equivariantly biholomorphic to $M^{0} \times_{\mathbb{C}^{\times}} \mathbb{C} P^{1} \rightarrow \hat{S}$ and $\hat{S}$ is a fibre product of flat projective unitary $\mathbb{C} P^{d_{0}}$ - and $\mathbb{C} P^{d_{\infty}}$-bundles over a Kähler manifold $S$ covered by $\prod_{a \in \mathcal{A}} S_{a}$.

If we assume that $\hat{S}=P\left(E_{0}\right) \times_{S} P\left(E_{\infty}\right) \rightarrow S$ for projectively-flat hermitian vector bundles $E_{0}, E_{\infty} \rightarrow S$, then these bundles can be chosen so that $M$ is $\mathbb{C}^{\times}$equivariantly biholomorphic to $P\left(E_{0} \oplus E_{\infty}\right) \rightarrow S$, and we therefore have $\bar{c}_{1}\left(E_{\infty}\right)-$ $\bar{c}_{1}\left(E_{0}\right)=\sum_{a \in \mathcal{A}}\left[\omega_{a} / 2 \pi\right]$, where $\bar{c}_{1}(E)=c_{1}(E) / \operatorname{rank} E$.

The final assumption of this theorem is automatic if $\pi_{1}(S)=1$, when $\hat{S}=$ $\mathbb{C} P^{d_{0}} \times S \times \mathbb{C} P^{d_{\infty}}$ and there is a line bundle $\mathcal{L} \rightarrow S$ with $c_{1}(\mathcal{L})=\sum_{a \in \mathcal{A}}\left[\omega_{a} / 2 \pi\right]$ such that $M$ is $\mathbb{C}^{\times}$-equivariantly biholomorphic to $P\left(\mathcal{O} \otimes \mathbb{C}^{d_{0}+1} \oplus \mathcal{L} \otimes \mathbb{C}^{d_{\infty}+1}\right) \rightarrow S$. 
We shall use this theorem as an Ansatz for constructing extremal Kähler metrics on projective bundles of the form $P\left(E_{0} \oplus E_{\infty}\right) \rightarrow S$. For this we shall use the following elementary computation [22, 25, 4].

Proposition 1. Let $g$ be a Kähler metric of the form (11) on $M^{0}$ and write $F(z)=$ $\Theta(z) p_{\mathrm{c}}(z)$ with $p_{\mathrm{c}}(z):=\prod_{a \in \hat{\mathcal{A}}}\left(1+x_{a} z\right)^{d_{a}}$. Then $g$ is extremal, with Scal $g$ a constant affine function of $z$, iff

- there is a polynomial $P$ of degree $\leq \# \hat{\mathcal{A}}+1$ such that

$$
F^{\prime \prime}(z)=\left(\prod_{a \in \hat{\mathcal{A}}}\left(1+x_{a} z\right)^{d_{a}-1}\right) P(z) ;
$$

- for all $a, \pm g_{a}$ has constant scalar curvature $S c a l_{ \pm g_{a}}= \pm 2 d_{a} s_{a}$ where

$$
P\left(-1 / x_{a}\right)=2 d_{a} s_{a} x_{a} \prod_{b \in \hat{\mathcal{A}} \backslash\{a\}}\left(1-\frac{x_{b}}{x_{a}}\right) .
$$

The metric $g$ then has constant scalar curvature iff $P$ has degree $\leq \# \hat{\mathcal{A}}$.

Compared to [4] [5], we have rescaled $F(z)$ and $p_{\mathrm{c}}(z)$ by $\prod_{a \in \hat{\mathcal{A}}} x_{a}^{d_{a}}$ : this is convenient as $p_{\mathrm{c}}(z)$ is then positive on $(-1,1)$. Thus $\Theta$ is positive on $(-1,1)$ if and only if $F$ is. Also if $\Theta(z)$ satisfies (3), then $F(z)=\Theta(z) p_{\mathrm{c}}(z)$ satisfies

$$
F( \pm 1)=0, \quad F^{\prime}( \pm 1)=\mp 2 p_{\mathrm{c}}( \pm 1) .
$$

The structure of the paper is as follows. In section 1 we study metrics of the form (11) and show that the conditions of Theorem 1 are sufficient for the compactification of such metrics on a projective bundle $M=P\left(E_{0} \oplus E_{\infty}\right) \rightarrow S$. We shall call metrics of this form (compatible with the given projective bundle structure on $M$ and local product structure on $S$ ) up to scale admissible. The projective bundle $M$ is similarly called an admissible bundle or manifold. We describe the admissible Kähler classes (i.e., those containing an admissible metric) in 1.2 and parameterize the admissible metrics in a given admissible class in 1.3 We end the section by computing in 1.4. the Lie algebra of Killing vector fields with zeros on $M$.

In section 2 we study extremal Kähler metrics in admissible Kähler classes and prove our first main result. In preparation for this, we consider what implications the existence of an extremal Kähler metric has for admissible bundles, Kähler classes and metrics. In 2.1, we apply the Matsushima-Lichnerowicz criterion [31, 36] to obtain information about the automorphism group of an admissible bundle when the base metric on $S$ is CSC. Then, in $₫ 2.2$ we compute the Futaki invariant $\mathfrak{F}_{\Omega}(K)$ of the vector field $K=J \operatorname{grad}_{q} z$ for any admissible Kähler class $\Omega$ on an admissible $2 m$-manifold $M$. Finally in $\$ 2.3$ we give a formula for the Mabuchi-Guan-Simanca (modified) K-energy functional on admissible Kähler metrics in $\Omega$ : it is determined by a polynomial $F_{\Omega}$ of degree $\leq m+2$, which we call the extremal polynomial (the degree $m+2$ coefficient being a nonzero multiple of $\mathfrak{F}_{\Omega}(K)$ ). Using the recent work of Chen-Tian [10, 11], we deduce that if $\Omega$ contains an extremal Kähler metric, then $F_{\Omega}$ must be nonnegative on $[-1,1]$.

In 2.4, we apply Proposition 10 to give a construction of admissible extremal Kähler metrics, unifying and generalizing work of Calabi, Guan, Hwang, HwangSinger and the fourth author [8, 22, 25, 26, 46]. This leads to the following result.

Theorem 2. Let $M=P\left(E_{0} \oplus E_{\infty}\right) \rightarrow S$ be an admissible manifold, where the base $S$ is a local Kähler product of CSC metrics $\left( \pm g_{a}, \pm \omega_{a}\right)$. Then there is an extremal Kähler metric in an admissible Kähler class $\Omega$ if and only if the extremal polynomial $F_{\Omega}$ is positive on $(-1,1)$. This condition always holds if $\Omega$ is 'sufficiently small'; 
if it does, there is an admissible extremal Kähler metric in $\Omega$, which is CSC if and only if the Futaki invariant $\mathfrak{F}_{\Omega}(K)$ vanishes (i.e., $F_{\Omega}$ has degree $\leq m+1$ ).

The admissible Kähler classes containing an extremal Kähler metric form a nonempty open subset of all such classes, and those containing a CSC Kähler metric form a real analytic hypersurface which is nonempty if $\bar{c}_{1}\left(E_{\infty}\right)-\bar{c}_{1}\left(E_{0}\right)$ is strictly indefinite (i.e., the Kähler forms $\pm \omega_{a}$ do not all have the same sign).

Here we say that an admissible Kähler class is sufficiently small if the $x_{a}(a \in \mathcal{A})$ are sufficiently small. Geometrically, this means that the base $S$ is large (low curvature) compared to the fibres (high curvature). Thus the above theorem asserts the existence of extremal Kähler metrics with curvature concentrated in the fibres (cf. [27, 40]). Note also that 'the' extremal Kähler metric in $\Omega$ (if it exists) is unique up to automorphism by Chen-Tian [10, 11].

In section 3 we present further existence and nonexistence results for extremal and CSC metrics by computing the extremal polynomial on various examples and testing its positivity on $(-1,1)$. In many of these examples, every Kähler class on $M$ is admissible (see Remark 2), and therefore Theorem 2 describes exactly which Kähler classes contain an extremal Kähler metric. This is the case when $M=P(\mathcal{O} \oplus \mathcal{L}) \rightarrow S$ is an admissible geometrically ruled complex surface; the extremal polynomial $F_{\Omega}(z)$ is then a quartic divisible by $1-z^{2}$. We thus obtain a complete resolution of the existence question for extremal Kähler metrics on these complex surfaces: the Kähler cone is a cone on an open interval $(a, b)$; the extremal Kähler metrics are precisely those of [7, 25, 46, which are admissible and locally cohomogeneity one, with Kähler classes parameterized by a cone on a subinterval $(a, c)$ and $c=b$ if and only if $S$ has genus 0 or 1. As observed in [2], this fills in the missing step in the complete classification of extremal Kähler metrics on geometrically ruled complex surfaces.

Our results provide a fertile testing ground for the conjectures relating extremal and CSC Kähler metrics to stability, and we explore this in section [4. In 4.2 we relate our results to those of Ross-Thomas [40] and Hong [27]: in particular, we show that there are CSC metrics on projective bundles $P(E) \rightarrow S$ for which $E$ is only (slope) polystable with respect one Kähler class on $S$ up to scale.

In 4.4. we relate Theorem 2 to the notions of K-polystability [14, 45] or relative K-polystability 44] for Kähler classes, which are conjectured to be equivalent to the existence of a CSC or extremal Kähler metric in a given class. Actually, to be precise, we use a closely related notion of (relative) slope K-polystability suggested by the work of Ross-Thomas [40, 41. Then, generalizing a calculation of G. Székelyhidi for ruled surfaces, we establish the following result.

Theorem 3. Let $\Omega$ be an admissible integral Kähler class on $M=P\left(E_{0} \oplus E_{\infty}\right) \rightarrow$ $S$, where $S$ is CSC. If $\Omega$ is slope K-polystable, it contains a CSC Kähler metric, and if it is slope $K$-polystable relative to $K=J \operatorname{grad}_{g} z$, it contains an extremal Kähler metric.

It is natural to ask if (relative) K-polystability in the sense of 14, 44 implies the existence of a CSC (or extremal) Kähler metric in $\Omega$. We find that this is true if $\operatorname{dim} S \leq 4$, but for $\operatorname{dim} S \geq 6$, we are only able to show that the extremal polynomial is positive on $(-1,1) \cap \mathbb{Q}$.

Before our work, it was believed that K-polystability implies slope K-polystability in general, but the proof in [40, 41] only shows that it implies slope K-semistability, the gap being closely related to the issue of positivity (versus nonnegativity) of the extremal polynomial at irrational points in $(-1,1)$. 
To show that this is a genuine problem, we end with some examples, on projective line bundles over a product of three Riemann surfaces, of integral admissible Kähler classes $\Omega$ such that $F_{\Omega}$ is positive on $(-1,1) \cap \mathbb{Q}$ but has an irrational repeated root in $(-1,1)$. We find these examples intriguing, since by Theorem [2] these Kähler classes do not contain an extremal Kähler metric so they should be unstable. However, despite being projective varieties, the degeneration that demonstrates this instability is not algebraic. While we cannot prove that there is no other (algebraic) test configuration which would detect this instability, it is difficult to imagine how such a test configuration could be constructed. Our results then suggest that the non-algebraic degenerations implicit in the use of slope K-polystability may be essential to relate stability to existence of CSC and extremal Kähler metrics.

We would like to thank Claude LeBrun for helpful comments and Richard Thomas for useful discussions concerning [40, 41].

\section{CONTEnTs}

\begin{tabular}{|c|}
\hline 1. Admissible bundles and Kähler metrics \\
\hline 1.1. Admissible proiective bundles \\
\hline 1.2. Admissible Kähler classes and canonical metrics \\
\hline 1.3. Admissible metrics \\
\hline 1.4. The isometrv Lie algebra \\
\hline 2. Admissible extremal Kähler metrics \\
\hline 2.1. Automorphisms and the Matsushima-Lichnerowicz obstruction \\
\hline 2.2. The Futaki invariant and extremal vector field \\
\hline 2.3. K-energy and the extremal polvnomial \\
\hline 2.4. A characterization of extremal admissible Kähler classes \\
\hline 3. Existence and nonexistence results for extremal Kähler metrics \\
\hline 3.1. Constructing admissible extremal Kähler metrics \\
\hline 3.2. Extremal Kähler metrics over a Riemann surface \\
\hline 3.3. Nonexistence of CSC Kähler metrics over a Hodge 4-manifold \\
\hline 3.4. CSC Kähler metrics over a product of two Riemann surfaces \\
\hline 4. K-stabilitv and admissible extremal Kähler metrics \\
\hline 4.1. Introduction to stabilitv \\
\hline 4.2. Stable bundles and CSC Kähler metrics \\
\hline 4.3. Extremal Kähler metrics and relative K-polvstabilitv \\
\hline 4.4. Relative K-polvstability of admissible projective bundles \\
\hline Appendix A. Relation to previous papers \\
\hline A.1. Summary of the classification \\
\hline A.2. A nonexistence result for order 2 extremal Kähler metrics \\
\hline Appendix B. Proof of Lemma $\overline{6}$ \\
\hline References \\
\hline
\end{tabular}

\section{Admissible BUndles AND KäHLER Metrics}

1.1. Admissible projective bundles. We use Theorem 1 (including the final assumption) as motivation for the class of compact complex manifold we will study. A projective bundle of the form $M=P\left(E_{0} \oplus E_{\infty}\right) \stackrel{p}{\rightarrow} S$ will be called admissible or an admissible manifold if:

- $S$ is a covered by a product $\tilde{S}=\prod_{a \in \mathcal{A}} S_{a}$ (for $\mathcal{A} \subset \mathbb{Z}^{+}$) of simply-connected Kähler manifolds $\left(S_{a}, \pm g_{a}, \pm \omega_{a}\right)$ of real dimensions $2 d_{a}$; 
- $E_{0}$ and $E_{\infty}$ are holomorphic projectively-flat hermitian vector bundles over $S$ of ranks $d_{0}+1$ and $d_{\infty}+1$ with $\bar{c}_{1}\left(E_{\infty}\right)-\bar{c}_{1}\left(E_{0}\right)=\left[\omega_{S} / 2 \pi\right]$ and $\omega_{S}=\sum_{a \in \mathcal{A}} \omega_{a}$.

The second condition (cf. 28]) means that we can fix hermitian metrics on $E_{0}$ and $E_{\infty}$ whose Chern connections have tracelike curvatures $\Omega_{0} \otimes I d_{E_{0}}$ and $\Omega_{\infty} \otimes I d_{E_{\infty}}$ satisfying $\Omega_{\infty}-\Omega_{0}=\sum_{a \in \mathcal{A}} \omega_{a}$. We normalize the induced fibrewise Fubini-Study metrics $\left(g_{0}, \omega_{0}\right)$ and $\left(-g_{\infty},-\omega_{\infty}\right)$ on $P\left(E_{0}\right)$ and $P\left(E_{\infty}\right)$ to have scalar curvatures $2 d_{0}\left(d_{0}+1\right)$ and $2 d_{\infty}\left(d_{\infty}+1\right)$.

We collect a few remarks and notations that we will use. We omit pullbacks by obvious projections in these remarks.

(i) We sometimes let the index $a$ take values in $\mathbb{N} \cup\{\infty\}$ by setting $d_{a}=0$ for $a \notin \mathcal{A} \cup\{0, \infty\}$ (so that $S_{a}$ is a point and $\omega_{a}=0$ ). This range will be assumed unless otherwise stated. We set $\hat{\mathcal{A}}:=\left\{a: d_{a}>0\right\}$ so that $\mathcal{A}=\hat{\mathcal{A}} \cap \mathbb{Z}^{+}$.

(ii) The pullbacks of $E_{0}$ and $E_{\infty}$ to $\tilde{S}$ are of the form $\mathcal{E}_{0} \otimes \mathbb{C}^{d_{0}+1}$ and $\mathcal{E}_{\infty} \otimes \mathbb{C}^{d_{\infty}+1}$, where $\mathcal{L}:=\mathcal{E}_{0}^{-1} \otimes \mathcal{E}_{\infty}=\bigotimes_{a \in \mathcal{A}} \mathcal{L}_{a}$ for line bundles $\mathcal{L}_{a} \rightarrow S_{a}$ with $c_{1}\left(\mathcal{L}_{a}\right)=\left[\omega_{a} / 2 \pi\right]$. (iii) $e_{0}:=P\left(E_{0} \oplus 0\right)$ and $e_{\infty}:=P\left(0 \oplus E_{\infty}\right)$ denote the 'zero' and 'infinity' subbundles of $M$, covered by $S_{0} \times \tilde{S}$ and $\tilde{S} \times S_{\infty}$, where $S_{0}=\mathbb{C} P^{d_{0}}$ and $S_{\infty}=\mathbb{C} P^{d_{\infty}}$.

(iv) The blow-up of $M$ along $e_{0} \cup e_{\infty}$ is $\hat{M}:=P(\mathcal{O} \oplus \hat{\mathcal{L}}) \stackrel{\hat{p}}{\rightarrow} \hat{S}$, where $\hat{S}=P\left(E_{0}\right) \times S$ $P\left(E_{\infty}\right) \rightarrow S$ and $\hat{\mathcal{L}}=\mathcal{O}(1)_{E_{0}} \otimes \mathcal{O}(-1)_{E_{\infty}}$, using the (fibrewise) hyperplane and tautological line bundles; we have $c_{1}(\hat{\mathcal{L}})=\left[\omega_{\hat{S}} / 2 \pi\right]$, where $\omega_{\hat{S}}=\sum_{a} \omega_{a}$. If $d_{0}>0$ or $d_{\infty}>0$ we say a blow-down occurs.

(v) $\hat{e}_{0}$ and $\hat{e}_{\infty}$ denote the zero and infinity sections of $\hat{M}$. The pullback of $\hat{\mathcal{L}}$ to $S_{0} \times \tilde{S} \times S_{\infty}$ is $\mathcal{L}_{0} \otimes \mathcal{L} \otimes \mathcal{L}_{\infty}$, where $\mathcal{L}_{0}=\mathcal{O}(1) \rightarrow S_{0}$ and $\mathcal{L}_{\infty}=\mathcal{O}(-1) \rightarrow S_{\infty}$.

(vi) $\hat{S}$ has a family of local Kähler product metrics $g_{\hat{S}}(z)$ with Kähler forms $z \omega_{\hat{S}}+$ $\sum_{a} \omega_{a} / x_{a}$ and we set $g_{\hat{S}}=g_{\hat{S}}(0)$. (Note that $g_{\hat{S}}$ is not compatible with $\omega_{\hat{S}}$ the latter is symplectic, but not a Kähler form in general.) We let $g_{S}(z)$ and $g_{S}=g_{S}(0)$ denote the induced local Kähler product metrics on $S$.

We summarize the set-up with the following diagram of bundles and a blow-up:

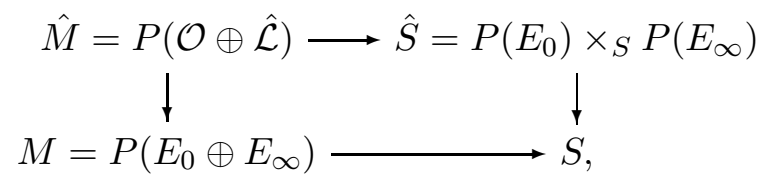

the universal cover (omitting pullbacks) of this diagram being:

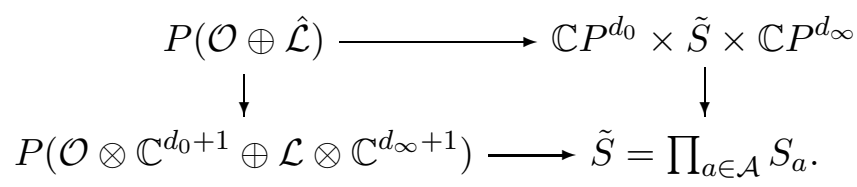

Remark 1. The existence of the line bundle $\hat{\mathcal{L}} \rightarrow \hat{S}$ with $c_{1}(\hat{\mathcal{L}})=\left[\omega_{\hat{S}} / 2 \pi\right]$ implies that $\omega_{\hat{S}}$ is integral in the sense that $\left[\omega_{\hat{S}} / 2 \pi\right]$ is in the image of $H^{2}(\hat{S}, \mathbb{Z})$ in $H^{2}(\hat{S}, \mathbb{R})$. When $\hat{S}$ is a global Kähler product (i.e., we can write $M=P\left(\mathcal{O} \otimes \mathbb{C}^{d_{0}+1} \oplus \mathcal{L} \otimes\right.$ $\left.\left.\mathbb{C}^{d_{\infty}+1}\right) \rightarrow S=\prod_{a \in \mathcal{A}} S_{a}\right)$ this integrality condition means that each $\omega_{a}$ is integral, i.e., the compact manifolds $\left(S_{a}, \pm g_{a}, \pm \omega_{a}\right)$ are Hodge.

We write $\omega_{a}=q_{a} \alpha_{a}$ for an integer $q_{a} \neq 0$, where $\alpha_{a}$ is a primitive integral Kähler form on $S_{a}$, so that $q_{a}$ is a nonzero integer with the same sign as $\left(g_{a}, \omega_{a}\right)$, and $q_{0}=1$ and $q_{\infty}=-1$. We now compare $\left[\omega_{a} / 2 \pi\right]$ to the first Chern class $c_{1}\left(\mathcal{K}_{a}^{-1}\right)=\left[\rho_{a} / 2 \pi\right]$ of the anticanonical bundle of $S_{a}$, by writing $\left[\rho_{a}\right]=p_{a}\left[\alpha_{a}\right]+\left[\rho_{a}\right]_{0}$, for a rational number $p_{a}$, where $\left[\rho_{a}\right]_{0} \cdot\left[\alpha_{a}\right]^{d_{a}-1}=0$. Since any line bundle $\mathcal{P}$ with first Chern class $\left[\alpha_{a} / 2 \pi\right]$ is ample, $\mathcal{P}^{d_{a}+1} \otimes \mathcal{K}_{a}$ is nef by a result of Fujita [20] (see also [12, 
Theorem 8.3]), from which it follows easily that $p_{a} \leq d_{a}+1$. If $S_{a}$ is a Riemann surface of genus $\mathbf{g}_{a}$, then $p_{a}=2\left(1-\mathbf{g}_{a}\right) \leq 2$.

We set $s_{a}=p_{a} / q_{a}$. When $\pm g_{a}$ is CSC, we have $S c a l_{ \pm g_{a}}= \pm 2 d_{a} s_{a}$, where the sign is that of $q_{a}$, so the scalar curvature of $\pm g_{a}$ has the same sign as $p_{a}$. Thus, in the case of a CSC Hodge manifold $S_{a}$, the Fujita inequality $p_{a} \leq d_{a}+1$ is (since $\left.\left|q_{a}\right| \geq 1\right)$ equivalent to $\operatorname{Scal}_{ \pm g_{a}} \leq 2 d_{a}\left(d_{a}+1\right)$.

The conditions of Theorem 1 are also sufficient for the compactification of metrics of the form (11) on an admissible projective bundle $M=P\left(E_{0} \oplus E_{\infty}\right) \rightarrow S$, where $z: M \rightarrow[-1,1]$ with $e_{0}=z^{-1}(1)$ and $e_{\infty}=z^{-1}(-1)$, and $\theta$ is a connection 1-form. Before discussing this, we introduce the Kähler classes to which they belong.

1.2. Admissible Kähler classes and canonical metrics. Suppose that $M=$ $P\left(E_{0} \oplus E_{\infty}\right) \rightarrow S$ is an admissible bundle. We say that a Kähler class $\Omega$ on $M$ is admissible if there are constants $x_{a}$, with $x_{0}=1, x_{\infty}=-1$, such that the pullback of $\Omega$ to $\hat{M}$ has the form

$$
\sum_{a}\left[\omega_{a}\right] / x_{a}+\hat{\Xi}
$$

up to scale, where the 2 -forms $\omega_{a}$ are viewed as pullbacks to $\hat{M}$ of the corresponding forms on $\hat{S}$ (induced by the local product Kähler structure $\prod_{a} S_{a}$ ) and $\hat{\Xi}$ is Poincaré dual to $2 \pi\left[\hat{e}_{0}+\hat{e}_{\infty}\right]$. Thus $\hat{\Xi}=2 \pi c_{1}(V \hat{M})$, where $V \hat{M}=\mathcal{O}(2)_{\mathcal{O} \oplus \hat{\mathcal{L}}} \otimes \hat{p}^{*} \hat{\mathcal{L}}$ and $\mathcal{O}(-1)_{\mathcal{O} \oplus \hat{\mathcal{L}}}$ is the (fibrewise) tautological bundle of $\hat{M}=P(\mathcal{O} \oplus \hat{\mathcal{L}})$. (The first Chern class $\left[\omega_{\hat{S}} / 2 \pi\right]$ of $\hat{\mathcal{L}}$ itself pulls back to $\hat{M}$ to give the Poincaré dual of $\left[\hat{e}_{0}-\hat{e}_{\infty}\right]$.)

It follows that admissible Kähler classes have the form

$$
\Omega=\sum_{a \in \mathcal{A}}\left[\omega_{a}\right] / x_{a}+\Xi
$$

up to scale, where the pullback of $\Xi$ to $\hat{M}$ is $\left[\omega_{0}\right]-\left[\omega_{\infty}\right]+\hat{\Xi}$. Since pullback to a blow-up is injective on cohomology, admissible Kähler classes on $M$ are uniquely determined by the parameters $x_{a}$.

If $(g, \omega)$ is any Kähler metric on $M$ of the form (11) on $M^{0}$, then we claim $\Omega=[\omega]$ is admissible. For this we first note that on $M^{0}$, the Kähler form $\omega$ is a linear combination $\sum_{a \in \mathcal{A}} \omega_{a} / x_{a}+\eta$, where

$$
\eta=(z+1) \omega_{0}+\sum_{a \in \mathcal{A}} z \omega_{a}+(z-1) \omega_{\infty}+d z \wedge \theta .
$$

Here $\omega_{0}, \omega_{\infty}$ and $\theta$ are defined only on $M^{0}$. However, for $a \in \mathcal{A}, \omega_{a}$ extends to a closed 2-form on $M$ (as a pullback from $S$ ), so $\eta$ is globally defined and closed on $M$ (since $\omega$ is). The pullback of $\eta$ to $\hat{M}$ may be written $\omega_{0}-\omega_{\infty}+\hat{\eta}$ with $\hat{\eta}=d(z \theta)$ on $M^{0}$, and since $\omega_{0}$ and $\omega_{\infty}$ are well-defined and closed on $\hat{M}$ (as pullbacks from $\hat{S})$, so is $\hat{\eta}$, and we easily see $^{1}$ that $[\hat{\eta}]=\hat{\Xi}$.

Observe that $\eta$ depends implicitly on the choice of metric $(g, \omega)$ on $M$ because the momentum map $z$ does. However, the above shows that the cohomology class $[\eta]$ is $\Xi$, independent of this choice. From this realisation of $\Xi$ it follows easily, by pulling back to $e_{0}$ and $e_{\infty}$, that for a cohomology class of the form $\sum_{a \in \mathcal{A}}\left[\omega_{a}\right] / x_{a}+\Xi$ to be a Kähler class, it is necessary that for $a \in \mathcal{A}, 0<\left|x_{a}\right|<1$ with the sign of $x_{a}$ such that $\omega_{a} / x_{a}$ is positive. Conversely, we claim that any cohomology class of

\footnotetext{
${ }^{1}$ On each fibre of $\hat{p}: \hat{M} \rightarrow \hat{S}, \hat{\eta} / 4 \pi$ integrates to 1 and so $[\hat{\eta} / 4 \pi]$ restricts to give the generator of $H^{2}\left(\hat{p}^{-1}(x), \mathbb{Z}\right)$. Hence by the Leray-Hirsch theorem, $H^{2}(\hat{M}, \mathbb{R})$ is generated by $[\hat{\eta}]$ and pullbacks from $S$. The restriction of $[\hat{\eta} / 2 \pi]$ to $\hat{e}_{0}$ is the first Chern class $\left[\omega_{\hat{S}} / 2 \pi\right]$ of $\hat{\mathcal{L}}$ (and the restriction to $\hat{e}_{\infty}$ is the first Chern class $\left[-\omega_{\hat{S}} / 2 \pi\right]$ of $\left.\hat{\mathcal{L}}^{-1}\right)$. Thus $[\hat{\eta} / 4 \pi]$ is a projective version of the Thom class of a vector bundle.
} 
this form (with $0<\left|x_{a}\right|<1$ and $\omega_{a} / x_{a}$ positive for $a \in \mathcal{A}$ ) is an admissible Kähler class and contains a Kähler metric of the form (11) on $M^{0}$ up to scale. To do this we construct a distinguished Kähler metric in each such class.

Let $r_{0}$ and $r_{\infty}$ be the norm functions induced by the hermitian metrics on $E_{0}$ and $E_{\infty}$. Then $z_{0}=\frac{1}{2} r_{0}^{2}$ and $z_{\infty}=\frac{1}{2} r_{\infty}^{2}$ are fibrewise momentum maps for the $U(1)$ actions given by scalar multiplication in $E_{0}$ and $E_{\infty}$, generated by $K_{0}$ and $K_{\infty}$. We equip $M$ with a fibrewise Fubini-Study metric $\left(g_{M / S}, \omega_{M / S}\right)$ : with our normalization of $g_{0}$ and $g_{\infty}$ each fibre is the Kähler quotient of the corresponding fibre of $E_{0} \oplus E_{\infty}$ by the diagonal $U(1)$ action at momentum level $z_{0}+z_{\infty}=2$; then on this momentum level the function $z=z_{0}-1=1-z_{\infty}$ descends to a fibrewise momentum map $M \rightarrow[-1,1]$ for the quotient $U(1)$ action.

We extend $\left(g_{M / S}, \omega_{M / S}\right)$ to $T M$ by requiring that the horizontal distribution of the induced connection on $M$ is degenerate. To obtain a nondegenerate metric, we then set

$$
g_{c}=\sum_{a \in \mathcal{A}} \frac{1+x_{a} z}{x_{a}} g_{a}+g_{M / S}, \quad \omega_{c}=\sum_{a \in \mathcal{A}} \frac{1+x_{a} z}{x_{a}} \omega_{a}+\omega_{M / S},
$$

where the $\left(g_{a}, \omega_{a}\right)$ are pulled back from $S ; g_{c}$ is then a positive definite Kähler metric with respect to the canonical complex structure of $M=P\left(E_{0} \oplus E_{\infty}\right)$ by the assumptions on the parameters $x_{a}$. We refer to $\left(g_{c}, \omega_{c}\right)$ as the canonical Kähler metric on $M$ in the given admissible Kähler class.

Lemma 1. For any $0<\left|x_{a}\right|<1(a \in \mathcal{A})$, the corresponding canonical Kähler metric on $M$ is of the form (10) on $M^{0}$, where $\Theta=\Theta_{c}$ and $\Theta_{c}(z)=1-z^{2}$.

Proof. The inverse image in $E_{0} \oplus E_{\infty}$ of $M^{0}=M \backslash\left(z^{-1}(-1) \cup z^{-1}(1)\right)$ may be viewed as an open subset of $\mathcal{O}(-1)_{E_{0}} \oplus \mathcal{O}(-1)_{E_{\infty}}$. Then $\left(g_{c}, \omega_{c}\right)$ is the Kähler quotient at momentum level $z_{0}+z_{\infty}=2$ of the metric

$$
\sum_{a} \frac{\left(1+x_{a}\right) z_{0}+\left(1-x_{a}\right) z_{\infty}}{2 x_{a}} g_{a}+\frac{d z_{0}^{2}}{2 z_{0}}+\frac{d z_{\infty}^{2}}{2 z_{\infty}}+2 z_{0} \theta_{0}^{2}+2 z_{\infty} \theta_{\infty}^{2}
$$

where $x_{0}=1, x_{\infty}=-1$, and $\theta_{0}, \theta_{\infty}$ are connection 1 -forms for the $U(1)$-line bundles $\mathcal{O}(-1)_{E_{0}}, \mathcal{O}(-1)_{E_{\infty}}$ with $\theta_{0}\left(K_{0}\right)=1=\theta_{\infty}\left(K_{\infty}\right), d \theta_{0}=-\omega_{0}+\Omega_{0}, d \theta_{\infty}=\omega_{\infty}+\Omega_{\infty}$.

If regard $M^{0}$ as an open subset of $\hat{M}=P(\mathcal{O} \oplus \hat{\mathcal{L}})$, then the diagonal action is generated by $K_{0}+K_{\infty}, \theta_{\infty}-\theta_{0}$ is basic and so induces a unitary connection $\theta$ (with respect to the quotient $U(1)$-action) on $\hat{\mathcal{L}}$ with $d \theta=\omega_{\hat{S}}$. Substituting $z_{0}=1+z$ and $z_{\infty}=1-z$ and performing the quotient yields (II) with $\Theta=\Theta_{c}$. (On each fibre over $\hat{S}$ this is the realization of $\mathbb{C} P^{1}$ as a Kähler quotient of $\mathbb{C}^{2}$.)

Remark 2. The existence of the canonical metric on $M$ shows there does exist a cohomology class $\Xi$ whose pullback to $\hat{M}$ is $\hat{\Xi}$. $\Xi$ is then unique, and the admissible Kähler classes form a family of dimension $\# \mathcal{A}+1$. If $b_{2}\left(S_{a}\right)=1$ for all $a$ and $b_{1}\left(S_{a}\right) \neq 0$ for at most one $a$, then every Kähler class on $M$ is admissible.

1.3. Admissible metrics. Let $M=P\left(E_{0} \oplus E_{\infty}\right) \rightarrow S$ be an admissible bundle and $\Omega$ an admissible Kähler class corresponding to parameters $x_{a}$. Then a Kähler metric in $\Omega$ is said to be admissible if it has the form (11) on $M^{0}$, up to scale, with respect to the given projective unitary bundle structure on $M$ and local Kähler product structure on $S$. According to Theorem 1 in order for a (scale of a) metric of the form (11) on $M^{0}$ to define an admissible Kähler metric on $M$, it is necessary that $\Theta$ is a smooth function on $[-1,1]$ satisfying (2)-(3). We now show that these conditions are also sufficient and provide a parameterization of admissible metrics. 
We first note that any metric of the form (11), where $\Theta$ is a smooth function on $[-1,1]$ satisfying (2)-(3), defines a smooth metric $g$ on $M$ compatible with the same symplectic form as the canonical Kähler metric $g_{c}$ in $\Omega$, provided that we take $z$ to be the momentum map and $\theta$ the connection 1-form of the canonical Kähler metric; then, using (3), we find that $g-g_{c}$ is smooth on $M$, and $g$ is positive definite on $M$ since it is on $M^{0}$ by (2) and $\omega$ is nondegenerate on $M$. (See [5, §1] for details.)

With this point of view, the smooth functions $\Theta$ on $[-1,1]$ satisfying (2)-(3) define a family of complex structures on $M$. However, we claim that there is an $S^{1}$-equivariant biholomorphism in the identity component of the diffeomorphism group between any two such complex structures, so that $\Theta$ parameterizes Kähler metrics compatible with the given (fixed) complex structure on $M$ whose Kähler forms belong to a given admissible Kähler class $\Omega$. This claim holds essentially because it is true for toric complex structures on $\mathbb{C} P^{1}$ (and for toric varieties in general), but for later use we need to make explicit the transformation of $M$ that relates the complex and symplectic points of view, following [24, 23, 14].

A key ingredient in this transformation is the notion of a symplectic potential of an admissible Kähler metric defined by $\Theta(z)$, which is a function $u(z)$ on $(-1,1)$ with $u^{\prime \prime}(z)=U(z)=1 / \Theta(z)$. Then

$$
u_{c}(z)=\frac{1}{2}((1-z) \log (1-z)+(1+z) \log (1+z)-2 \log 2)
$$

is the unique symplectic potential for the canonical Kähler metric $\left(g_{c}, J_{c}\right)$ given by $\Theta_{c}(z)=(1-z)(1+z)$, which satisfies $u_{c}( \pm 1)=0$. We can extend this description to all admissible Kähler metrics compatible with $\omega$, thanks to the following lemma, which is an easy application of l'Hôpital's rule and Taylor's Theorem.

Lemma 2. A smooth function $\Theta(z)=1 / U(z)$ satisfies (3) if and only if $U(z)-$ $U_{c}(z)$ is smooth on $[-1,1]$. Then $U(z) / U_{c}(z)$ is positive and smooth on $[-1,1]$.

On $M^{0}$ the symplectic potential $u(z)$ of an admissible Kähler metric is closely related to a Kähler potential of $\omega$ with respect to $J$ by a fibrewise Legendre transform (see [4, 24]) over $\hat{S}$. Indeed, if we put

$$
y=u^{\prime}(z), \quad h(y)=-u(z)+y z
$$

then $d_{J}^{c} y=\theta$ and $d d_{J}^{c} h(y)=\omega-\sum_{a} \omega_{a} / x_{a}$ on $\left(M^{0}, J\right)^{2}$. Let $y_{c}, h_{c}\left(y_{c}\right)$ denote the corresponding quantities associated to $u_{c}$. There are local 1-forms $\alpha$ on $\hat{S}$ such that $\theta=d t+\alpha$, where $t: M^{0} \rightarrow \mathbb{R} / 2 \pi \mathbb{Z}$ is locally defined up to an additive constant on each fibre. Since $\exp (y+i t)$ and $\exp \left(y_{c}+i t\right)$ give $\mathbb{C}^{\times}$-coordinates on the fibres, there is a $U(1)$-equivariant fibre-preserving diffeomorphism $\Psi$ of $M^{0}$ over $\hat{S}$ with

$$
\Psi^{*} y=y_{c}, \quad \Psi^{*} t=t, \quad \text { and hence } \quad \Psi^{*} J=J_{c} .
$$

As $J_{c}$ and $J$ are integrable complex structures, $\Psi$ extends to a $U(1)$-equivariant diffeomorphism of $M$ leaving fixed any point on $e_{0} \cup e_{\infty}$ (since it is fibre preserving).

Put $\tilde{\omega}:=\Psi^{*} \omega$. Then $\tilde{\omega}$ is a Kähler form on $\left(M, J_{c}\right)$ which (we claim) belongs to the same cohomology class $\Omega$ as $\omega$. Indeed, on $M^{0}$ we have

$$
\tilde{\omega}-\omega=d d_{J_{c}}^{c}\left(h\left(y_{c}\right)-h_{c}\left(y_{c}\right)\right)
$$

since $d d_{J_{c}}^{c} h\left(y_{c}\right)=\Psi^{*} d d_{J}^{c} h(y)=\tilde{\omega}-\sum_{a} \omega_{a} / x_{a}$, so the following implies the claim.

Lemma 3. The function $h\left(y_{c}\right)-h_{c}\left(y_{c}\right)$ is smooth on $M$.

\footnotetext{
${ }^{2}$ It follows that if $\pm H_{a}$ is a local Kähler potential for $\pm \omega_{a}$ and $\tilde{u}=u(z)-\sum_{a}\left(1+x_{a} z\right) H_{a} / x_{a}$, then $\tilde{y}=\partial \tilde{u} / \partial z$ is pluriharmonic and $\tilde{h}=-\tilde{u}+\tilde{y} z$ is a local Kähler potential for $\omega$ on $\left(M^{0}, J\right)$.
} 
Proof. Since $\Psi$ is a diffeomorphism with $\Psi^{*} y=y_{c}$, this holds if and only if $h(y)-$ $h_{c}(y)$ is smooth on $M$. We already know that $h(y)-h_{c}\left(y_{c}\right)=-\left(u(z)-u_{c}(z)\right)+$ $z\left(u^{\prime}(z)-u_{c}^{\prime}(z)\right)$ is smooth (by Lemma 2) so it suffices to show that $h_{c}(y)-h_{c}\left(y_{c}\right)$ is smooth on $M$. However, knowing $u_{c}$ explicitly, we calculate

$$
h_{c}(y)-h_{c}\left(y_{c}\right)=-\frac{1}{2}\left(\log \left(\frac{1-\tilde{z}}{1-z}\right)+\log \left(\frac{1+\tilde{z}}{1+z}\right)\right),
$$

where $\tilde{z}:=\Psi^{*} z$ is the momentum map of $\tilde{\omega}=\Psi^{*} \omega$; since $\Psi$ is $S^{1}$-equivariant and fixes $e_{0} \cup e_{\infty}$, it follows that $\tilde{z}$, viewed as a function of $z$, satisfies $\tilde{z}( \pm 1)= \pm 1$; moreover, since both $\tilde{z}$ and $z$ are momentum maps of the same $U(1)$ action on $M$ (and are therefore Morse-Bott functions with the same critical sets), we must have $\tilde{z}^{\prime}( \pm 1) \neq 0$. Thus $h_{c}(y)-h_{c}\left(y_{c}\right)$ is smooth on $M$.

Hence the moduli space $\mathcal{K}_{\omega}^{\mathrm{adm}}$ of admissible metrics in $\Omega=[\omega]$ is identified with the space of smooth functions $\Theta$ on $[-1,1]$ satisfying (2) -(3) or equivalently with $\left\{u \in C^{0}([-1,1]): u-u_{c} \in C^{\infty}([-1,1]), u( \pm 1)=0\right.$ and $u^{\prime \prime}>0$ on $\left.(-1,1)\right\}$.

1.4. The isometry Lie algebra. For a compact Kähler manifold $(M, g)$, we denote by $\mathfrak{i}_{0}(M, g)$ the Lie algebra of all Killing vector fields with zeros. Since $M$ is compact this is equivalently the Lie algebra of all hamiltonian Killing vector fields.

Proposition 2. Let $g$ be an admissible metric on $M=P\left(E_{0} \oplus E_{\infty}\right) \stackrel{p}{\rightarrow} S$ and equip $S$ and $\hat{S} \rightarrow S$ with the metrics $g_{S}, g_{\hat{S}}$ induced by $\sum_{a} g_{a} / x_{a}$ on $\prod_{a} S_{a}$. Let $\mathfrak{z}(K, g)$ be the centralizer in $\mathfrak{i}_{0}(M, g)$ of the Killing vector field $K=J \operatorname{grad}_{g} z$.

Then $\mathfrak{z}(K, g)$ is the direct sum of $\mathfrak{i}_{0}\left(\hat{S}, g_{\hat{S}}\right)$ and the span of $K$ in such a way that $p_{*}: \mathfrak{i}_{0}(M, g) \rightarrow \mathfrak{i}_{0}\left(S, g_{S}\right)$ is induced by the natural surjection $\mathfrak{i}_{0}\left(\hat{S}, g_{\hat{S}}\right) \rightarrow \mathfrak{i}_{0}\left(S, g_{S}\right)$.

Proof. Let $X$ be a holomorphic vector field on $\hat{S}$ which is hamiltonian with respect to $\omega_{h}:=\sum_{a} \omega_{a} / x_{a}$; then the projection $X_{a}$ of $X$ onto the distribution $\mathcal{H}_{a}$ (induced by $T S_{a}$ on the universal cover $\prod_{a} S_{a}$ of $\hat{S}$ ) is a Killing vector field with zeros, so $\iota_{X_{a}} \omega_{h}=-d f_{a}$ for some function $f_{a}$ (with integral zero). Thus $\sum_{a} f_{a} x_{a}$ is a hamiltonian for $X$ with respect to the symplectic form $\omega_{\hat{S}}=\sum_{a} \omega_{a}$ : since this is the curvature $d \theta$ of the connection on $M^{0}, X$ lifts to a holomorphic vector field $\tilde{X}=X_{H}+\left(\sum_{a} f_{a} x_{a}\right) K$ on $M^{0}$, which is hamiltonian with potential $\sum_{a}\left(1+x_{a} z\right) f_{a}$ and commutes with $K$. Here $X_{H}$ is the horizontal lift to $M^{0}$ with respect to $\theta$. $\tilde{X}$ and its potential extend to $M$ since $M \backslash M^{0}$ has codimension $\geq 2$ and $\tilde{X}$ has zeros.

Conversely any element of $\mathfrak{z}(K, g)$ pulls back to a holomorphic vector field $V$ on $\hat{M}$. The projection of $V$ to the normal bundle $\hat{p}^{*} T \hat{S}$ of $\hat{p}: \hat{M} \rightarrow \hat{S}$ is holomorphic hence constant on the $\mathbb{C} P^{1}$ fibres by Liouville's Theorem (the normal bundle is trivial on each fibre), so $V$ is projectable; it maps to zero iff it comes from a multiple of $K$. This gives a projection to $\mathfrak{i}_{0}\left(\hat{S}, g_{\hat{S}}\right)$ splitting the inclusion just defined.

\section{Admissible extremal KäHLer metrics}

2.1. Automorphisms and the Matsushima-Lichnerowicz obstruction. On any compact Kähler manifold $(M, g)$ the Lie algebra $\mathfrak{h}(M)$ of holomorphic vector fields lies in an exact sequence:

$$
0 \rightarrow \mathfrak{h}_{0}(M) \rightarrow \mathfrak{h}(M) \rightarrow H^{1}(M, \mathbb{R})^{*}
$$

where $\mathfrak{h}_{0}(M)$ is the ideal of holomorphic vector fields with zeros, which is the Lie algebra of the reduced automorphism group $H_{0}(M) \subset \operatorname{Aut}_{0}(M)$, the connected component of the kernel of the Albanese map $\operatorname{Aut}_{0}(M) \rightarrow H^{1}(M, \mathbb{R})^{*} / H_{1}(M, \mathbb{Z})$. The Matsushima-Lichnerowicz Theorem 31, 36] says that if $g$ is CSC, $\mathfrak{h}_{0}(M)$ is the complexification of the Lie algebra $\mathfrak{i}_{0}(M, g)$ of hamiltonian Killing vector fields and 
$\mathfrak{h}(M)=\mathfrak{a}(M) \oplus \mathfrak{h}_{0}(M)$ where $\mathfrak{a}(M)$ is the central subalgebra of parallel vector fields: thus $\mathfrak{h}(M)$ is reductive. This condition on $\mathfrak{h}(M)$ is often called the MatsushimaLichnerowicz obstruction to the existence of CSC Kähler metrics on $(M, J)$.

Let us consider the special case of geometrically ruled complex manifolds $M=$ $P(E)$ where $p: P(E) \rightarrow S$ and $E$ is a holomorphic vector bundle of rank $r+1$ over a compact Kähler $2 d$-manifold $\left(S, h, \omega_{h}\right)$. Thus, $M$ is a kählerian $2 m$-manifold where $m=r+d$ : for instance, $\left[\mathcal{O}(1)_{E}\right]+k\left[p^{*} \omega_{h}\right]$ is a Kähler class for $k \gg 1$, where $\mathcal{O}(-1)_{E}$ is the (fibrewise) tautological line bundle of $P(E)$.

The projection of any holomorphic vector field $V \in \mathfrak{h}(M)$ to the normal bundle $p^{*} T S$ is constant on each fibre, so $V$ descends to a holomorphic vector field $p_{*} V \in$ $\mathfrak{h}(S)$. Since $p_{*}\left[V_{1}, V_{2}\right]=\left[p_{*} V_{1}, p_{*} V_{2}\right]$, we have an exact sequence of Lie algebras

$$
0 \rightarrow \mathfrak{h}_{S}(M) \rightarrow \mathfrak{h}_{0}(M) \rightarrow \mathfrak{h}_{0}(S),
$$

where $\mathfrak{h}_{S}(M)$ is the subspace of $\mathfrak{h}(M)$ of holomorphic vector fields tangent to the fibres of $p$ (which have zeros). Obviously $\mathfrak{h}_{S}(M)=H^{0}(S, \mathfrak{s l}(E))$ is the Lie algebra of holomorphic vector fields preserving the $\mathbb{C} P^{r}$-fibres of $p: P(E) \rightarrow S$. Since an ideal in a reductive Lie algebra is reductive, we obtain the following weaker (but often more useful) version of the Matsushima-Lichnerowicz obstruction.

Proposition 3. Let $M=P(E) \rightarrow S$ be a geometrically ruled complex manifold which admits a CSC Kähler metric. Then $\mathfrak{h}_{S}(M)$ must be reductive.

The following elementary result yields a simple application of this criterion.

Proposition 4. Let $M=P\left(E_{0} \oplus E_{1} \oplus \cdots \oplus E_{\ell}\right)$, where $E_{j}$ are holomorphic vector bundles on a compact kählerian manifold $S$ such that $H^{0}\left(S, \mathfrak{g l}\left(E_{j}\right)\right)$ is reductive and $H^{0}\left(S, \operatorname{Hom}\left(E_{j}, E_{i}\right)\right)=0$ for all $i<j$. Then $\mathfrak{h}_{S}(M)$ is reductive iff $H^{0}\left(S, \operatorname{Hom}\left(E_{i}, E_{j}\right)\right)=0$ for all $i<j$.

Proof. Any element of the Lie algebra $\mathfrak{h}_{S}(M)=H^{0}(S, \mathfrak{s l}(E))$ may be represented as an $(\ell+1) \times(\ell+1)$ matrix $\left(a_{i j}\right)$ with $a_{i j} \in H^{0}\left(S, \operatorname{Hom}\left(E_{i}, E_{j}\right)\right)$. By assumption, this matrix is upper-triangular. The strictly upper-triangular matrices form a nilpotent ideal $\mathfrak{n}$ and if this is zero, $\mathfrak{h}_{S}(M)$ is clearly reductive. Conversely, taking commutators with elements of the form $I d_{E_{i}} / \operatorname{rank} E_{i}-I d_{E_{j}} / \operatorname{rank} E_{j} \in H^{0}(S, \mathfrak{s l}(E))$, we see that $\mathfrak{n} \subseteq\left[\mathfrak{h}_{S}(M), \mathfrak{h}_{S}(M)\right]$. Hence if $\mathfrak{n} \neq 0,\left[\mathfrak{h}_{S}(M), \mathfrak{h}_{S}(M)\right]$ is not semisimple, i.e., $\mathfrak{h}_{S}(M)$ is not reductive.

Corollary 1. Let $M=P(E)$ where $E=\mathcal{L}_{0} \oplus \mathcal{L}_{1} \oplus \cdots \oplus \mathcal{L}_{r}$ is a direct sum of holomorphic line bundles over a Riemann surface $\Sigma$ of genus $\mathbf{g}$. If $\mathbf{g} \geq 2$ and $\operatorname{deg} \mathcal{L}_{i}-\operatorname{deg} \mathcal{L}_{j}>\mathbf{g}-1$ for some $0 \leq i, j \leq r$ then $M$ admits no CSC Kähler metric. If $\mathbf{g} \leq 1$, then $M$ admits a CSC Kähler metric if and only if $\operatorname{deg} \mathcal{L}_{i}=\operatorname{deg} \mathcal{L}_{j}$ for all $i, j$ (i.e., $\mathcal{L}_{0} \oplus \mathcal{L}_{1} \oplus \cdots \oplus \mathcal{L}_{r}$ is a polystable vector bundle).

Proof. We can assume without loss that $E=\mathcal{E}_{0} \otimes \mathbb{C}^{r_{0}} \oplus \mathcal{E}_{1} \otimes \mathbb{C}^{r_{1}} \oplus \cdots \oplus \mathcal{E}_{\ell} \otimes \mathbb{C}^{r_{\ell}}$ with $\operatorname{deg} \mathcal{E}_{i} \leq \operatorname{deg} \mathcal{E}_{j}$ and $\mathcal{E}_{i} \not \mathcal{E}_{j}$ for $i<j$. The Kodaira vanishing theorem then implies $H^{0}\left(\Sigma, \mathcal{E}_{j}^{-1} \otimes \mathcal{E}_{i}\right)=0$ for any $i<j$, and we may apply Proposition 4 By assumption, $\operatorname{deg} \mathcal{E}_{\ell}-\operatorname{deg} \mathcal{E}_{0}>\max (0, \mathbf{g}-1)$, and so $\operatorname{dim} H^{0}\left(\Sigma, \mathcal{E}_{0}^{-1} \otimes \mathcal{E}_{\ell}\right)>0$ by Riemann-Roch. Hence $\mathfrak{h}_{\Sigma}(M)$ is not reductive, and there is no CSC Kähler metric on $M$. The converse when $\mathbf{g} \leq 1$ follows from Narasimhan-Seshadri 38.

Remark 3. The assumptions of Proposition 4 hold if $E_{1}, \ldots E_{\ell}$ are projectivelyflat hermitian vector bundles such that the slopes $\mu\left(E_{j}\right):=\bar{c}_{1}\left(E_{j}\right) \cup \Omega^{d-1}$, with respect to some Kähler class $\Omega$ on $S(\operatorname{dim} S=2 d)$, satisfy $\mu\left(E_{i}\right)<\mu\left(E_{j}\right)$ for $i<j$. Indeed in this case $\mathfrak{g l}\left(E_{j}\right)$ is a flat hermitian bundle and $H^{0}\left(S, \mathfrak{g l}\left(E_{j}\right)\right)$ is the space 
of parallel sections of $\mathfrak{g l}\left(E_{j}\right)^{3}$, which is a complexification of the space of parallel sections of $\mathfrak{u}\left(E_{j}\right)$, hence a reductive Lie algebra. The slope condition then ensures $H^{0}\left(S, \operatorname{Hom}\left(E_{j}, E_{i}\right)\right)=0$ for all $i<j$ by a theorem of Kobayashi [28].

In general, the condition that $\mathfrak{h}_{S}(M)$ is reductive does not $\operatorname{imply} \mathfrak{h}_{0}(M)$ is. However it does if $p_{*}: \mathfrak{h}_{0}(M) \rightarrow \mathfrak{h}_{0}(S)$ is surjective and $\mathfrak{h}_{0}(S)$ is reductive. This obviously holds if $\mathfrak{h}_{0}(S)=0$. It also holds if $\left(S, g_{S}\right)$ is CSC and there is a metric $g$ on $M$ such that $p_{*}$ is a surjection from $\mathfrak{i}_{0}(M, g)$ to $\mathfrak{i}_{0}\left(S, g_{S}\right)$. This is true for admissible bundles by Proposition 2

Proposition 5. Let $\Omega$ be an admissible Kähler class on $M=P\left(E_{0} \oplus E_{\infty}\right) \rightarrow S$ where the local product metric $g_{S}$ on $S$ is $C S C$. Then the admissible metrics in $\Omega$ are invariant under a common maximal compact connected subgroup of $H_{0}(M)$, and $\mathfrak{h}_{0}(M)$ is reductive iff $H^{0}\left(S, \operatorname{Hom}\left(E_{0}, E_{\infty}\right)\right)=0=H^{0}\left(S, \operatorname{Hom}\left(E_{\infty}, E_{0}\right)\right)$. This latter condition holds if $\bar{c}_{1}\left(E_{\infty}\right)-\bar{c}_{1}\left(E_{0}\right)$ is strictly indefinite.

Proof. Let $g$ be an admissible Kähler metric on $M$. We know that $\mathfrak{h}(S)$ is reductive and $\mathfrak{i}_{0}\left(S, g_{S}\right)$ is a maximal compact subalgebra of $\mathfrak{h}_{0}(S)$, and by Proposition 2 both $p_{*}: \mathfrak{h}_{0}(M) \rightarrow \mathfrak{h}_{0}(S)$ and $p_{*}: \mathfrak{i}_{0}(M, g) \rightarrow \mathfrak{i}_{0}\left(S, g_{S}\right)$ are surjective.

To show that the Lie algebra $\mathfrak{i}_{0}(M, g)$ is a maximal compact subalgebra of $\mathfrak{h}_{0}(M)$, it therefore suffices to show that $\mathfrak{i}_{0}(M, g) \cap \mathfrak{h}_{S}(M)$ is a maximal compact subalgebra of $\mathfrak{h}_{S}(M)$. Since $\bar{c}_{1}\left(E_{\infty}\right)-\bar{c}_{1}\left(E_{0}\right)=\omega_{S}$, we can certainly arrange that $\mu\left(E_{\infty}\right)-\mu\left(E_{0}\right)$ is nonzero by the choice of a Kähler class on $S$. Then, by Remark 3 , we are under the hypotheses of Proposition [4 and, as in its proof, we have that $\mathfrak{h}_{S}(M)$ is the direct sum of the reductive centralizer of $K$ and a nilpotent ideal $\mathfrak{n}$ in $\left[\mathfrak{h}_{S}(M), \mathfrak{h}_{S}(M)\right]$. The result now follows easily from Proposition 2 and Remark 3

As noted above, $\mathfrak{h}_{0}(M)$ is reductive iff $\mathfrak{h}_{S}(M)$ is. By Proposition 4 the latter happens iff both $H^{0}\left(S, \operatorname{Hom}\left(E_{0}, E_{\infty}\right)\right)=0=H^{0}\left(S, \operatorname{Hom}\left(E_{\infty}, E_{0}\right)\right)$. This indeed holds if $\bar{c}_{1}\left(E_{\infty}\right)-\bar{c}_{1}\left(E_{0}\right)$ is strictly indefinite (by the vanishing theorem of Kobayashi 28 as in Remark 3), since we can then choose Kähler classes on $S$ such that the corresponding slopes have $\mu\left(E_{\infty}\right)-\mu\left(E_{0}\right)$ with either sign.

Since $S$ is a local Kähler product, it is CSC iff the factors $S_{a}(a \in \mathcal{A})$ in the universal cover are CSC.

2.2. The Futaki invariant and extremal vector field. On a compact Kähler $2 m$-manifold $(M, J, g, \omega)$, recall that the (normalized) Futaki invariant of a real holomorphic vector field with zeros $V=J \operatorname{grad}_{g} f+\operatorname{grad}_{g} h$ is defined by

$$
\mathfrak{F}_{\omega}(V)=\left(\int_{M} \mu_{g} \int_{M}(f+i h) S c a l_{g} \mu_{g}-\int_{M} S c a l_{g} \mu_{g} \int_{M}(f+i h) \mu_{g}\right) / \operatorname{Vol}(M)^{2},
$$

where $\mu_{g}=\omega^{m} / m$ ! is the volume form of $g$. Futaki [17] showed that this complex number is independent of the choice of metric in the Kähler class $\Omega=[\omega]$, and that the map $\mathfrak{F}_{\Omega}: \mathfrak{h}_{0}(M) \rightarrow \mathbb{C}$ is a character on $\mathfrak{h}_{0}(M)$. $\mathfrak{F}_{\Omega}$ is closely related to the Futaki-Mabuchi extremal vector field $K_{\Omega}:=J \operatorname{grad}_{g} \operatorname{pr}_{g} S c a l_{g}$ of $(M, J, \Omega, G)$ where $G$ is a maximal compact connected subgroup of $H_{0}(M)$ and $\operatorname{pr}_{g}$ is the $L_{2}$-projection onto the space of Killing potentials with respect to any $G$-invariant metric $g$ in $\Omega$ : Futaki and Mabuchi [18] showed that $K_{\Omega}$ is independent of this choice. Clearly $\mathfrak{F}_{\Omega}$ and $K_{\Omega}$ vanish if $\Omega$ contains a CSC metric. Calabi [8] showed that if $\mathfrak{F}_{\Omega}$ vanishes

\footnotetext{
${ }^{3}$ This is a standard Bochner argument, as in 28. Alternatively, note that the pullback of $\mathfrak{g l}\left(E_{j}\right)$ to the universal cover of $S$ is trivialized by parallel sections, and apply the open mapping theorem: the pullback of a holomorphic section of $\mathfrak{g l}\left(E_{j}\right)$ has closed bounded image in this trivialization.
} 
then any extremal Kähler metric in $\Omega$ is a CSC metric, but the vanishing of $\mathfrak{F}_{\Omega}$ does not suffice in general for the existence of a CSC metric in $\Omega$.

Let $\Omega$ be an admissible Kähler class on $M=P\left(E_{0} \oplus E_{\infty}\right) \rightarrow S$ and suppose in addition that for $a \in \mathcal{A}, \pm g_{a}$ is a CSC Kähler metric with scalar curvature $S c a l_{ \pm g_{a}}= \pm 2 d_{a} s_{a}$. Let $p_{\mathrm{c}}(t)=\prod_{a}\left(1+x_{a} t\right)^{d_{a}}$ and define $\alpha_{r}=\int_{-1}^{1} p_{\mathrm{c}}(t) t^{r} d t$ and

$$
\beta_{r}:=p_{\mathrm{c}}(1)+(-1)^{r} p_{\mathrm{c}}(-1)+\int_{-1}^{1}\left(\sum_{a} \frac{d_{a} s_{a} x_{a}}{1+x_{a} t}\right) p_{\mathrm{c}}(t) t^{r} d t .
$$

We now compute the Futaki invariant $\mathfrak{F}_{\Omega}(K)$ of $K=J \operatorname{grad}_{g} z$ and show that $K_{\Omega}$ is essentially $\mathfrak{F}_{\Omega}(K) K$, where $G$ the maximal compact connected subgroup of $H_{0}(M)$ of Proposition 5 preserving admissible Kähler metrics in $\Omega$. $\mathfrak{F}_{\Omega}(K)$ will reappear in the next paragraph as the leading coefficient of a polynomial associated with $\Omega$.

Proposition 6. Suppose $M$ is admissible over a $C S C$ base and $\Omega$ is an admissible Kähler class with admissible metric $g$. Then $\mathfrak{F}_{\Omega}(K)=2\left(\alpha_{0} \beta_{1}-\alpha_{1} \beta_{0}\right) / \alpha_{0}^{2}$. Also the $L_{2}$-projection of $S_{c a l}$ orthogonal to the space of Killing potentials is

$$
\mathrm{Scal}_{g}+\mathrm{Az}+B
$$

where $A$ and $B$ are given by

$$
\begin{aligned}
& A \alpha_{1}+B \alpha_{0}=-2 \beta_{0} \\
& A \alpha_{2}+B \alpha_{1}=-2 \beta_{1} .
\end{aligned}
$$

(Since $\alpha_{0} \alpha_{2}>\alpha_{1}^{2}$, this system has a unique solution for $A, B$.) In particular the extremal vector field of $(\Omega, G)$ is $K_{\Omega}=-A K=2\left(\alpha_{0} \beta_{1}-\alpha_{1} \beta_{0}\right) K /\left(\alpha_{0} \alpha_{2}-\alpha_{1}^{2}\right)$.

Proof. We may rescale $\Omega$ so that an admissible metric $(g, \omega)$ in $\Omega$ is of the form (11). We then have

$$
\mu_{g}=\frac{\omega^{m}}{m !}=p_{\mathrm{c}}(z)\left(\bigwedge_{a} \frac{\left(\omega_{a} / x_{a}\right)^{d_{a}}}{d_{a} !}\right) \wedge d z \wedge \theta,
$$

where $m=1+\sum_{a} d_{a}$ is the complex dimension of $M$. Thus

$$
\begin{aligned}
\int_{M} \mu_{g} & =2 \pi \operatorname{Vol}\left(S, \prod_{a} \frac{\omega_{a}}{x_{a}}\right) \alpha_{0}(=\operatorname{Vol}(M)), \\
\int_{M} z \mu_{g} & =2 \pi \operatorname{Vol}\left(S, \prod_{a} \frac{\omega_{a}}{x_{a}}\right) \alpha_{1}=\operatorname{Vol}(M) \alpha_{1} / \alpha_{0},
\end{aligned}
$$

where $\operatorname{Vol}\left(S, \prod_{a} \frac{\omega_{a}}{x_{a}}\right)=\prod_{a} \operatorname{Vol}\left(S_{a}, \frac{\omega_{a}}{x_{a}}\right)$ in the case when $S$ is a global product.

The scalar curvature of $(M, g)$ is given by

$$
S c a l_{g}=\sum_{a} \frac{2 d_{a} s_{a} x_{a}}{1+x_{a} z}-\frac{F^{\prime \prime}(z)}{p_{\mathrm{c}}(z)}
$$

where $F(z)=\Theta(z) p_{\mathrm{c}}(z)$ (see e.g. [4, (79)]). We thus calculate

$$
\begin{array}{rl}
\int_{M} z & S c a l_{g} \mu_{g}=2 \pi \operatorname{Vol}\left(S, \prod_{a} \frac{\omega_{a}}{x_{a}}\right) \int_{-1}^{1}\left(\left(\sum_{a} \frac{2 d_{a} s_{a} x_{a}}{1+x_{a} z}\right) p_{\mathrm{c}}(z)-F^{\prime \prime}(z)\right) z d z \\
= & 2 \pi \operatorname{Vol}\left(S, \prod_{a} \frac{\omega_{a}}{x_{a}}\right)\left(\int_{-1}^{1}\left(\sum_{a} \frac{2 d_{a} s_{a} x_{a}}{1+x_{a} z}\right) p_{\mathrm{c}}(z) z d z-\left[z F^{\prime}(z)-F(z)\right]_{-1}^{1}\right) \\
& =2 \operatorname{Vol}(M) \beta_{1} / \alpha_{0},
\end{array}
$$

where we integrate by parts, then impose the boundary conditions (3). Similarly,

$$
\int_{M} S c a l_{g} \mu_{g}=2 \operatorname{Vol}(M) \beta_{0} / \alpha_{0}
$$


and the first claim follows.

For the second claim note that the above integral formulae imply $S_{c a l}+A z+B$ is orthogonal to the Killing potentials 1,z if and only if (9) holds. By the form of $S c a l_{g}$, the fact that the $s_{a}$ are constant, and Proposition 2] the result follows.

Note that the above expression for $\mathfrak{F}_{\Omega}(K)$ is manifestly independent of the choice of a smooth function $\Theta(z)$ satisfying (B), as it should be according to the general theory [17. Indeed, as we have already discussed in 91.3 , these smooth functions $\Theta(z)$ define Kähler metrics within the same Kähler class.

2.3. K-energy and the extremal polynomial. Given a complex $2 m$-manifold $(M, J)$, a maximal compact connected subgroup $G$ of $H_{0}(M)$ and a Kähler class $\Omega$, denote by $\mathcal{M}_{\Omega}$ the infinite dimensional Fréchet space of Kähler metrics in $\Omega$ and let $\mathcal{M}_{\Omega}^{G}$ be the subspace of $G$-invariant Kähler metrics in $\Omega$. Following Guan [23] and Simanca [42, consider the map

$$
g \mapsto \operatorname{pr}_{g}^{\perp} S c a l_{g} \mu_{g},
$$

where $\operatorname{pr}_{g}^{\perp}$ is the $L_{2}$-projection orthogonal to the space of Killing potentials. This can be viewed (by integration) as a 1-form $\sigma$ on $\mathcal{M}_{\Omega}^{G}$, which turns out to be closed. Therefore for any $\omega_{0} \in \Omega$, there exists a unique functional $E_{\omega_{0}}^{G}: \mathcal{M}_{\Omega}^{G} \rightarrow \mathbb{R}$ with

$$
d E_{\omega_{0}}^{G}=-\sigma,
$$

$E_{\omega_{0}}^{G}\left(\omega_{0}\right)=0$. Note that changing the base point $\omega_{0} \in \mathcal{M}_{\Omega}$ would change $E_{\omega_{0}}^{G}$ by an additive constant. We refer to $E_{\omega_{0}}^{G}$ as the (modified) K-energy: it agrees with the Mabuchi K-energy [33] when $G$ is trivial.

By definition, it is clear that the critical points of $E_{\omega_{0}}^{G}$ are exactly the extremal Kähler metrics in $\mathcal{M}_{\Omega}^{G}$, since $\sigma=0$ means that $S c a l_{g}$ is a Killing potential. Note that by the Calabi Theorem [7], any extremal Kähler metric $g \in \mathcal{M}_{\Omega}$ belongs to $\mathcal{M}_{\Omega}^{G}$ with $G=\operatorname{Isom}_{0}(M, g) \cap H_{0}(M)$.

Building on earlier work by Bando-Mabuchi [6], Chen [9], Donaldson [15] and others, Chen and Tian have established the following uniqueness result and necessary condition for existence of an extremal Kähler metric.

Theorem 4. 10, 11] Extremal Kähler metrics in $\mathcal{M}_{\Omega}$ are unique up to automorphism and any extremal Kähler metric in $\mathcal{M}_{\Omega}^{G}$ realizes the absolute minimum of $E_{\omega_{0}}^{G}\left(\right.$ for any $\left.\omega_{0} \in \mathcal{M}_{\Omega}^{G}\right)$. In particular, if $\mathcal{M}_{\Omega}^{G}$ contains an extremal Kähler metric, then $E_{\omega_{0}}^{G}$ is bounded from below.

Now let $M$ be an admissible projective bundle over a CSC base as in the previous paragraph. We want to obtain a formula for the K-energy as a functional acting on $\mathcal{K}_{\omega}^{\mathrm{adm}}$, where $\omega$ is fixed, so we need to use the description given in 1.3 which shows how $\mathcal{K}_{\omega}^{\mathrm{adm}}$ is embedded into $\mathcal{M}_{\Omega}^{G}$, in which the complex structure is fixed.

This description shows that if $u_{t}(z)$ is a path of symplectic potentials in $\mathcal{K}_{\omega}^{\mathrm{adm}}$, then the smooth functions $h_{t}\left(y_{c}\right)-h_{c}\left(y_{c}\right)$ define a path $\omega+d d_{J_{c}}^{c}\left(h_{t}\left(y_{c}\right)-h_{c}\left(y_{c}\right)\right)$ in $\mathcal{M}_{\Omega}$, where $h_{t}$ are introduced by

$$
h_{t}\left(y_{t}(z)\right)=-u_{t}(z)+y_{t} z, \quad y_{t}=u_{t}^{\prime}(z)
$$

so that with $z=y_{t}^{-1}\left(y_{c}\right)$ we have

$$
h_{t}\left(y_{c}\right)=-u_{t}\left(y_{t}^{-1}\left(y_{c}\right)\right)+y_{t}^{-1}\left(y_{c}\right) y_{c} .
$$


Differentiating with respect to $t$, we get for the corresponding vector fields $\dot{u} \in$ $T_{g}\left(\mathcal{K}_{\omega}^{\mathrm{adm}}\right)$ and $\dot{h} \in T_{\omega}\left(\mathcal{M}_{\Omega}^{G}\right)$, the relation (cf. [23, 14]):

$$
\dot{h}=-\dot{u} \text {. }
$$

Hence we obtain the following symplectic version of the (modified) K-energy.

Lemma 4. The K-energy $E_{\omega}^{G}$, restricted to the space of admissible Kähler metrics in $\Omega$ and viewed as a function on the space of symplectic potentials, is determined uniquely up to an additive constant by the formula

$$
\left(d E_{\omega}^{G}\right)_{g}[\dot{u}]=\int_{M}\left(\operatorname{pr}_{g}^{\perp} S c a l_{g}\right) \dot{u} \mu_{g}
$$

where $\operatorname{pr}_{g}^{\perp}$ denotes the $L_{2}$-projection orthogonal to the space of Killing potentials.

Consider an admissible metric $g$ in $\Omega$ corresponding to the function $\Theta(z)=$ $F(z) / p_{\mathrm{c}}(z)$. Since the base $S$ is CSC we have, by Proposition [6 $\operatorname{pr}_{g}^{\perp} S c a l_{g}=S c a l_{g}+$ $A z+B$, with $A$ and $B$ given by (9) and $S c a l_{g}$ by (10).

Lemma 5. There is a unique smooth function $F_{\Omega}$ on $[-1,1]$ with

$$
F_{\Omega}^{\prime \prime}(z)=\left(A z+B+\sum_{a} \frac{2 d_{a} s_{a} x_{a}}{1+x_{a} z}\right) p_{\mathrm{c}}(z)
$$

and $F_{\Omega}( \pm 1)=0 . \quad F_{\Omega}$ satisfies (6) and is a polynomial of degree $\leq m+2$, the coefficient of $z^{m+2}$ being a nonzero multiple of $A$.

Proof. There is clearly a unique solution to (11) with $F_{\Omega}( \pm 1)=0$. One easily checks, using (9) that the solution is

$$
(1+z) p_{\mathrm{c}}(1)+(1-z) p_{\mathrm{c}}(-1)+\int_{-1}^{1}\left(\frac{1}{2}(A t+B)+\sum_{a} \frac{d_{a} s_{a} x_{a}}{1+x_{a} t}\right) p_{\mathrm{c}}(t)|z-t| d t .
$$

The derivative of this function is

$$
p_{\mathrm{c}}(1)-p_{\mathrm{c}}(-1)+\int_{-1}^{1}\left(\frac{1}{2}(A t+B)+\sum_{a} \frac{d_{a} s_{a} x_{a}}{1+x_{a} t}\right) p_{\mathrm{c}}(t) \operatorname{sign}(z-t) d t,
$$

which gives the formulae for $F_{\Omega}^{\prime}( \pm 1)$ in (6), using the first equation of (9).

The motivation for this lemma is that now $\operatorname{Scal}_{g}+A z+B=\left(F_{\Omega}^{\prime \prime}(z)-F^{\prime \prime}(z)\right) / p_{\mathrm{c}}(z)$. Furthermore, $F$ and $F_{\Omega}$ satisfy the same boundary conditions (6).

Proposition 7. Let $\Omega$ be an admissible Kähler class on an admissible bundle over a CSC base. Then the K-energy restricted to the space of admissible Kähler metrics $\mathcal{K}_{\omega}^{\mathrm{adm}}$ is (up to an additive constant) a positive multiple of the functional

$$
\mathcal{E}_{g_{c}}: u(z) \mapsto \int_{-1}^{1} F_{\Omega}(z)\left(u^{\prime \prime}(z)-u_{c}^{\prime \prime}(z)\right) d z-\int_{-1}^{1} p_{c}(z) \log \left(\frac{u^{\prime \prime}(z)}{u_{c}^{\prime \prime}(z)}\right) d z .
$$

Proof. $\mathcal{E}_{g_{c}}$ is well-defined by Lemma 2 and its gradient is

$$
\left(d \mathcal{E}_{g_{c}}\right)_{g}[\dot{u}]=\int_{-1}^{1} F_{\Omega}(z) \dot{u}^{\prime \prime}(z) d z-\int_{-1}^{1} p_{c}(z) \frac{\dot{u}^{\prime \prime}(z)}{u^{\prime \prime}(z)} d z=\int_{-1}^{1}\left(F_{\Omega}(z)-F(z)\right) \dot{u}^{\prime \prime}(z) d z .
$$

Integrating twice by parts, using the fact that $F$ and $F_{\Omega}$ both satisfy (6), and multiplying by $2 \pi \operatorname{Vol}\left(S, \prod_{a} \omega_{a} / x_{a}\right)$, we obtain $\int_{M}\left(S c a l_{g}+A z+B\right) \dot{u} \mu_{g}$. 
Remark 4. It is worth noticing that (by (3) ) the integral

$$
\int_{-1}^{1}\left(F_{\Omega}(z) u^{\prime \prime}(z)-p_{c}(z) \log u^{\prime \prime}(z)\right) d z
$$

exists for any admissible Kähler metric in $\Omega$ with symplectic potential $u(z)$, giving a definition of K-energy on $\mathcal{K}_{\omega}^{\mathrm{adm}}$ which is independent of a choice of reference metric.

Corollary 2. If there is an extremal Kähler metric in $\Omega$, then $F_{\Omega} \geq 0$ on $[-1,1]$.

Proof. If there is an extremal Kähler metric in $\Omega$, then by Theorem 4 [10, 11], the K-energy is bounded from below. We now apply an argument from [14: take any nonnegative smooth function $f(z)$ with $\operatorname{supp}(f) \subset(-1,1)$ and consider the sequence $u_{k}(z)$ with $u_{k}^{\prime \prime}(z)=u_{c}^{\prime \prime}(z)+k f(z)$ of symplectic potentials (cf. Lemma 2) for admissible Kähler metrics. We therefore get

$$
\mathcal{E}_{g_{c}}\left(u_{k}\right)=-\int_{-1}^{1} p_{c}(z) \log \left(1+k \frac{f(z)}{u_{c}^{\prime \prime}(z)}\right) d z+k \int_{-1}^{1} F_{\Omega}(z) f(z) d z .
$$

This will tend to $-\infty$ if $\int_{-1}^{1} F_{\Omega}(z) f(z) d z<0$ for some $f$.

In the next paragraph, where we complete the proof of Theorem [2 we shall show that positivity of $F_{\Omega}$ on $(-1,1)$ is a necessary and sufficient condition for the existence of an extremal Kähler metric in $\Omega$.

Definition 1. Let $\Omega$ be an admissible Kähler class on $M$. Then the polynomial $F_{\Omega}$ constructed above will be called the extremal polynomial of $\Omega$.

2.4. A characterization of extremal admissible Kähler classes. In this paragraph we prove Theorem 2 in three steps. First, if the extremal polynomial $F_{\Omega}$ of an admissible Kähler class $\Omega$ is positive on $(-1,1)$, we construct an admissible extremal Kähler metric in $\Omega$ by adapting an argument of Guan and Hwang (cf. 22, 25, 26]): we discuss their work further in the next section. Second, we extend the continuity argument of [46] to prove the existence of admissible extremal Kähler metrics for $\Omega$ sufficiently small. Third, we use Corollary 2 , the uniqueness result of ChenTian [10, 11] and an argument from [46] to show that an extremal Kähler metric in $\Omega$ is admissible up to automorphism. Hence we deduce that the existence of an extremal Kähler metric in $\Omega$ implies that $F_{\Omega}$ is positive on $(-1,1)$.

We begin with the construction. By Proposition 11 an admissible metric (1) is extremal exactly when for each $a \in \hat{\mathcal{A}}, \pm g_{a}$ is a CSC Kähler metric with $S c a l_{ \pm g_{a}}=$ $\pm 2 d_{a} s_{a}$ and (44)-(5) hold for a polynomial $P$ of degree $\leq N+1$, where $N=\# \hat{\mathcal{A}}$. The metric $g$ is CSC iff $P$ has degree $\leq N$.

We have seen that the boundary conditions (3) imply (6) and the converse clearly holds if $p_{\mathrm{c}}( \pm 1) \neq 0$ (i.e., $\left.d_{0}=0=d_{\infty}\right)$. However, if $g$ is extremal, then (4)-(15) imply that $F^{\prime \prime}(z)=p_{\mathrm{c}}^{\prime}(z) \Upsilon(z)$ with $\Upsilon(-1)=2\left(d_{0}+1\right)$ if $d_{0}>0$ and $\Upsilon(1)=-2\left(d_{\infty}+1\right)$ if $d_{\infty}>0$ (because of the normalization of the Fubini-Study metrics on $S_{0}$ and $S_{\infty}$ ). Hence, by (6),$F^{\prime}(z)=p_{\mathrm{c}}(z) \Psi(z)$ with $\Psi(-1)=2\left(d_{0}+1\right)$ and $\Psi(1)=-2\left(d_{\infty}+1\right)$, and $\Theta( \pm 1)=0$. Now by l'Hôpital's rule, $\Theta^{\prime}( \pm 1)=\mp 2$. Hence for extremal Kähler metrics, the boundary conditions (3) are equivalent to (6).

In summary, to obtain a globally defined admissible extremal metric on a projective bundle $P\left(E_{0} \oplus E_{\infty}\right) \rightarrow S$, we need, for CSC Kähler metrics $\left( \pm g_{a}, \pm \omega_{a}\right)$ satisfying $\bar{c}_{1}\left(E_{\infty}\right)-\bar{c}_{1}\left(E_{0}\right)=\sum_{a}\left[\omega_{a} / 2 \pi\right]$, to solve (4) and (5) for a polynomial $F$ (of degree $\leq m+2$ ) which satisfies (6) and is positive on $(-1,1)$.

For an admissible Kähler class $\Omega$ on $M$, we claim that (4)-(15) and the boundary conditions (6) have a unique solution for $F$, given by the extremal polynomial $F_{\Omega}$. 
Proposition 8. Let $M=P\left(E_{0} \oplus E_{\infty}\right) \rightarrow S$ be an admissible $2 m$-manifold, where $S$ is CSC. Then for any admissible Kähler class $\Omega$ on $M$, the extremal polynomial $F_{\Omega}$ is the unique polynomial $F$ of degree $\leq m+2$ satisfying (44)-(15) and (6).

Proof. An admissible Kähler class on $M$ is specified by parameters $x_{a}$ such that $x_{0}=1, x_{\infty}=-1$ and otherwise $0<\left|x_{a}\right|<1$ with $\omega_{a} / x_{a}$ positive. We write $S c a l_{ \pm g_{a}}= \pm 2 d_{a} s_{a}$. Equation (5) can be solved for a degree $N-1$ polynomial $P_{0}$ by Lagrange interpolation, i.e.,

$$
P_{0}(z)=\sum_{a} 2 d_{a} s_{a} x_{a} \prod_{b \in \hat{\mathcal{A}}}\left(1+x_{b} z\right)
$$

and then we can write the general degree $N+1$ solution as

$$
P(z)=P_{0}(z)+(A z+B) \prod_{a \in \hat{\mathcal{A}}}\left(1+x_{a} z\right)=\left(A z+B+\sum_{a} \frac{2 d_{a} s_{a} x_{a}}{1+x_{a} z}\right) \prod_{a \in \hat{\mathcal{A}}}\left(1+x_{a} z\right)
$$

so that

$$
F^{\prime \prime}(z)=\left(A z+B+\sum_{a} \frac{2 d_{a} s_{a} x_{a}}{1+x_{a} z}\right) p_{\mathrm{c}}(z) .
$$

Integrating $F^{\prime \prime}(z)$ and $z F^{\prime \prime}(z)$ on $[-1,1]$, (6) now implies that $A, B$ satisfy (9). Hence $F=F_{\Omega}$ is the unique solution.

Remark 5. An alternative approach is to solve the initial value problem (at $z=$ -1 ) for $F(z)$. The boundary conditions at $z=1$ then show that $A, B$ satisfy (9). This gives another formula for the extremal polynomial:

$$
F_{\Omega}(z)=2(z+1) p_{\mathrm{c}}(-1)+\int_{-1}^{z}\left(A t+B+\sum_{a} \frac{2 d_{a} s_{a} x_{a}}{1+x_{a} t}\right) p_{\mathrm{c}}(t)(z-t) d t
$$

where $A$ and $B$ are given (as usual) by (9).

Proposition 8 shows that the existence of an admissible extremal Kähler metric in $\Omega$ is equivalent to the positivity of the extremal polynomial $F_{\Omega}$ on $(-1,1)$. Since the leading coefficient is a nonzero multiple of $A$, Proposition [6 shows that such a metric will be CSC iff the Futaki invariant $\mathfrak{F}_{\Omega}(K)$ vanishes.

Remark 6. Since $F_{\Omega}$ depends continuously (in fact analytically) on the admissible Kähler class, it is positive on $(-1,1)$ for an open subset of such classes. This observation fits in with the general stability result of LeBrun and Simanca 30].

We now show that $F_{\Omega}$ is positive on $(-1,1)$ for sufficiently small $\Omega$.

Proposition 9. Let $M=P\left(E_{0} \oplus E_{\infty}\right) \rightarrow S$ be admissible, where $S$ is a local Kähler product of CSC metrics $\left( \pm g_{a}, \pm \omega_{a}\right)$. Then there is a nonempty open subset of admissible Kähler classes on $M$ which contain an (admissible) extremal Kähler metric of positive scalar curvature. The admissible Kähler classes containing a CSC metric form a real analytic hypersurface which is nonempty if $\bar{c}_{1}\left(E_{\infty}\right)-\bar{c}_{1}\left(E_{0}\right)$ is strictly indefinite over $S$ (i.e., the definite forms $\omega_{a}$ do not all have the same sign).

Proof. As we noted in Remark 6 , the extremal polynomial $F_{\Omega}$ is positive on $(-1,1)$ for an open subset of admissible Kähler classes. It remains to see that this open subset is nonempty and to find the CSC metrics in the family. For this, we study the behaviour of $F_{\Omega}$ near $x_{a}=0$ for all $a \in \mathcal{A}$. 
Lemma 6. The coefficients $A$ and $B$ defined by (9), as functions of $x_{a}(a \in \mathcal{A})$ for $\left|x_{a}\right|$ small are given by

$$
\begin{aligned}
A=- & 2\left(2+d_{0}+d_{\infty}\right) \sum_{a \in \mathcal{A}} d_{a} x_{a}+O\left(x^{2}\right) \\
B=- & \left(1+d_{0}+d_{\infty}\right)\left(2+d_{0}+d_{\infty}\right) \\
& -2 \sum_{a \in \mathcal{A}} d_{a} s_{a} x_{a}+2\left(d_{0}-d_{\infty}\right) \sum_{a \in \mathcal{A}} d_{a} x_{a}+O\left(x^{2}\right)
\end{aligned}
$$

where $O\left(x^{2}\right)$ is shorthand for $\sum_{a, b \in \mathcal{A}} O\left(x_{a} x_{b}\right)$.

The proof is given in Appendix B. It follows that in the limit $x_{a} \rightarrow 0$ for all $a \in \mathcal{A}$ (which does not give a Kähler class), $F_{\Omega}^{\prime \prime}(z) /(1+z)^{d_{0}}(1-z)^{d_{\infty}}$ is given by

$$
-\left(1+d_{0}+d_{\infty}\right)\left(2+d_{0}+d_{\infty}\right)+\frac{2 d_{0}\left(d_{0}+1\right)}{1+z}+\frac{2 d_{\infty}\left(d_{\infty}+1\right)}{1-z} .
$$

If $d_{0}=0$ and $d_{\infty}=0$, this is negative on $(-1,1)$ and $F_{\Omega}$ is convex. By (6), for some $\varepsilon>0, F_{\Omega}$ is positive and increasing on $(-1,-1+\varepsilon)$ and concave if $d_{0}>0$, while it is positive and decreasing on $(1-\varepsilon, 1)$ and concave if $d_{\infty}>\infty$. By (15), for sufficiently small $\Omega, F_{\Omega}$ does not have enough inflection points to have a zero on $(-1,1)$ and so it is positive there. Hence the set of admissible Kähler classes containing an admissible extremal Kähler metric is nonempty. Since $z \in[-1,1]$, we see that for $x_{a}$ sufficiently small, the scalar curvature $-A z-B$ of $g$ is positive.

Now the Futaki invariant $\mathcal{F}_{\Omega}(K) \sim A$ is a rational function of $x_{a}, a \in \mathcal{A}$, so the CSC metrics form a real analytic hypersurface. It is then clear from (13) that if $x_{a}$ occur with both signs, $A$ has nonconstant sign for small $x_{a}$.

(Using the Matsushima-Lichnerowicz criterion, the CSC existence result in this proposition provides an alternative proof of Proposition 5.)

Proposition 10. Let $M$ be an admissible projective bundle over a CSC base. Then if an admissible Kähler class on $M$ contains an extremal Kähler metric, this extremal Kähler metric is admissible up to automorphism.

Proof. Consider the set $U$ of admissible Kähler classes that contain an extremal Kähler metric invariant under the maximal compact subgroup $G$ of $H_{0}(M)$ defined in Proposition 5. By LeBrun-Simanca [30, $U$ is open in the set of all admissible Kähler classes. Suppose there is some admissible class $\Omega_{0}$ (with parameters $x_{a}^{0}$ ) which contains an inadmissible extremal metric $g_{0}$; by the Calabi Theorem, we can assume that $g_{0}$ is $G$-invariant, i.e., $\Omega_{0} \in U$. By LeBrun-Simanca [30, this implies that in all Kähler classes sufficiently close to $\Omega_{0}$ there are $G$-invariant extremal Kähler metrics close to $g_{0}$ (in suitable Sobolev spaces; by the Sobolev embedding theorem this also holds in the $C^{\ell}(M)$ topology, for any $\left.\ell>0\right)$. We have two cases:

- there is an open neighbourhood of $\Omega_{0}$ in $U$ for which the extremal polynomial is not positive on $(-1,1)$;

- there are admissible extremal Kähler metrics in classes arbitrarily close to $\Omega_{0}$.

The first case contradicts the existence of admissible extremal Kähler metrics on $M$ for sufficiently small $\Omega$, i.e., the positivity of the extremal polynomial. Indeed, for such $\Omega, F_{\Omega}(z)$ has at most two inflection points in $(-1,1)$ by (15), and an easy case by case analysis (according to whether $d_{0}, d_{\infty}$ are zero or positive) then shows that $Q_{\Omega}(z):=F_{\Omega}(z) /(1+z)^{d_{0}}(1-z)^{d_{\infty}}$, as a polynomial in $z$, has simple roots. However, by assumption, for all $\Omega$ in some open neighbourhood of $\Omega_{0}$, the extremal polynomial $F_{\Omega}$ is nonnegative (by Corollary 2) but not positive on $(-1,1)$, so $Q_{\Omega}(z)$ has zero discriminant. Since it is analytic in $\Omega$, it is identically zero, a contradiction. 
In the second case we apply instead the uniqueness result [10, 11] for extremal Kähler metrics, as in [46]. Let $\Omega_{k}$ be a sequence of admissible Kähler classes (with parameters $x_{a}^{k}$ ) which converges to $\Omega_{0}$ (i.e., $x_{a}^{k}$ converges to $x_{a}^{0}$ for all $a$ ) and such that $\Omega_{k}$ contains an admissible extremal Kähler metric $\tilde{g}_{k}$ which is not CSC. By LeBrun-Simanca 30, it follows that for $k \gg 1$ there are $G$-invariant extremal Kähler metrics $g_{k} \in \Omega_{k}$ which converge to $g_{0}$ in the $C^{2}(M)$ topology. By ChenTian [10, 11], $g_{k}$ is the pullback of $\tilde{g}_{k}$ by an automorphism $\Psi_{k}$ of $\left(M, J, \Omega_{k}, G\right)$. We now claim that $g_{0}$ is the pullback by an automorphism of an admissible extremal Kähler metric in $\Omega_{0}$, completing the proof.

To prove the claim, we use the theory of hamiltonian 2-forms of order 1 from [4, 5]. Since $\tilde{g}_{k}$ admits such a 2 -form with $S^{1}$ action generated by $K$, so does $g_{k}$ (by Proposition [6] $K$ is a nonzero multiple of the extremal vector field of $\left(\Omega_{k}, G\right)$ and so is preserved by $\left.\Psi_{k}\right)$. Now if $(g, \omega)$ is any Kähler metric on $M$ for which $K=$ $J \operatorname{grad}_{g} z$ generates an isometric $S^{1}$ action, then it follows from [5] that this action comes from a hamiltonian 2-form if and only if it is rigid (meaning that $g(K, K)$ depends only on $z$ ) and semisimple (meaning that for any regular value $z_{0}$ of $z$, the $z$-derivative at $z=z_{0}$ of the family of Kähler quotient metrics $g_{\hat{S}}(z)$ on the complex quotient $\hat{S}$ is parallel and diagonalizable with respect to $\left.g_{\hat{S}}\left(z_{0}\right)\right)$. Thus, the $S^{1}$ action generated by $K$ is rigid and semisimple with respect to $g_{k}$, hence also with respect to $g_{0}$ by continuity, so that $g_{0}$ itself admits a hamiltonian 2-form of order 1 with $S^{1}$ action generated by $K$.

We now apply Theorem 1 and Proposition 1 to $g_{0}$ : it follows that $g_{0}$ is adapted to the bundle structure of $M=P\left(E_{0} \oplus E_{\infty}\right) \rightarrow S$ and induces a CSC Kähler metric $g_{\hat{S}}{ }^{0}$ on $\hat{S}=P\left(E_{0}\right) \times{ }_{S} P\left(E_{\infty}\right)$, and a connection 1 -form $\theta_{0}$ on the principal $\mathbb{C}^{\times}$bundle over $\hat{S}$ (whose total space is identified with $M^{0}$ ), such that the $(1,1)$-form $d \theta_{0}=: \omega_{\hat{S}}^{0}$ is parallel and diagonalizable with respect to $g_{\hat{S}}{ }^{0}$. Then (by ChernWeil theory) we have $\left[\omega_{\hat{S}}^{0}\right]=\left[\omega_{\hat{S}}\right]$. It follows that the Kähler form of $g_{\hat{S}}{ }^{0}$ is in the cohomology class $\sum_{a}\left[\omega_{a}\right] / x_{a}^{0}$, since, as explained in 1.2 this cohomology class is determined by the admissible class $\Omega_{0}$, which can be uniquely written as the sum of the 'projective Thom class' $\hat{\Xi}$, of $\hat{M}=P(\mathcal{O} \oplus \hat{L}) \rightarrow \hat{S}$ and a pullback from $\hat{S}$.

Now by Chen-Tian [10, 11] again, there is an automorphism $\psi$ of $\hat{S}$ with $g_{\hat{S}}=$ $\psi^{*} g_{\hat{S}}{ }^{0}$, since these are CSC Kähler metrics in the same Kähler class. In fact, the proof of [10, 11] essentially shows that any two extremal metrics in a given Kähler class can be connected by a geodesic in the space of Kähler potentials, and therefore $\psi$ can be chosen in the reduced (connected) automorphism group $H_{0}(\hat{S}) \subseteq$ $\operatorname{Aut}_{0}(\hat{S})$ (see e.g. 23, 21]); in particular, such a $\psi$ acts trivially on cohomology. By Proposition 2 there is a fibre-preserving $S^{1}$-equivariant automorphism $\Psi$ of $\hat{M}=P(\mathcal{O} \oplus \hat{L}) \rightarrow \hat{S}$, which induces $\psi$ on $\hat{S}$. Thus, $\Psi$ preserves the $\mathbb{C}^{\times}$-bundle structure of $M^{0} \rightarrow \hat{S}$ and sends the connection 1-form $\theta_{0}$ to a connection 1-form $\tilde{\theta}_{0}$ with curvature $d \tilde{\theta}_{0}=\psi^{*} \omega_{\hat{S}}^{0} \in\left[\omega_{\hat{S}}^{0}\right]=\left[\omega_{\hat{S}}\right]$; now since $\psi^{*} \omega_{\hat{S}}^{0}$ and $\omega_{\hat{S}}$ are both parallel (and therefore harmonic) with respect to $\psi^{*} g_{\hat{S}}{ }^{0}=g_{\hat{S}}$, Hodge theory implies they are equal. We can therefore send $\tilde{\theta}_{0}$ to $\theta$ via a bundle isomorphism.

Thus we have constructed (on $M^{0}$ and hence, by a standard extension argument, everywhere) an automorphism sending $g_{0}$ to an admissible extremal Kähler metric in $\Omega_{0}$, as required.

Theorem 2 follows from Propositions 810. 


\section{Existence AND NONEXistence RESUlts FOR EXtREmal KäHLER METRICS}

In this section we use Theorem 2 to construct explicit examples of extremal Kähler metrics. We also obtain some nonexistence results for CSC Kähler metrics.

3.1. Constructing admissible extremal Kähler metrics. We begin with a root counting argument due to Hwang [25] and Guan [22] which gives a complete construction when the base $S$ is a local Kähler product of nonnegative CSC Kähler metrics (in fact Hwang and Guan only considered the case that $S$ has constant nonnegative eigenvalues of the Ricci tensor, but the proof is no different in general, and the idea to weaken this hypothesis is already explored in 26]).

Proposition 11. Suppose that $M=P\left(E_{0} \oplus E_{\infty}\right) \rightarrow S$ is admissible where $S$ is a local Kähler product of nonnegative CSC metrics. Then every admissible Kähler class contains an (admissible) extremal Kähler metric.

Proof. By the boundary conditions $F_{\Omega}$ is positive, and increasing or decreasing, on $(-1,-1+\varepsilon)$ or $(1-\varepsilon, 1)$ respectively, for some $\varepsilon>0$. Suppose it is not positive on $(-1,1)$. Then it has at least two maxima, one minimum and two inflection points on $(-1,1)$. It follows that $P$ has at least two roots in $(-1,1)$.

Let $y_{1} \leq \cdots \leq y_{Q}$ and $z_{1} \leq \cdots \leq z_{R}(Q, R \geq 0)$ denote the roots (counted with multiplicity) of $P$ in $[1, \infty)$ and $(-\infty,-1]$ respectively, and put $y_{0}=1, y_{Q+1}=\infty$, $z_{0}=-\infty, z_{R+1}=-1$. We order $\left\{x_{a}: a \in \hat{\mathcal{A}}\right\}$ as

$$
-1 \leq x_{a_{1}}<\cdots<x_{a_{J}}<0<x_{a_{J+1}}<\cdots<x_{a_{N}} \leq 1
$$

(for some $0 \leq J \leq N)$ so that $g_{a_{j}}$ is negative definite (hence with $s_{a_{j}}$ nonpositive) for $j \leq J$ and positive definite (hence with $s_{a_{j}}$ nonnegative) for $j \geq J+1$.

Therefore by (5), for each $0 \leq q \leq Q$, there is at most one $x_{a_{j}}$ with $y_{q} \leq$ $-1 / x_{a_{j}}<y_{q+1}$, so that $Q+1 \geq J$ with equality iff there is exactly one $x_{a_{j}}$ in each such interval. Similarly, for each $0 \leq r \leq R$, there is at most one $x_{a_{j}}$ with $z_{r}<-1 / x_{a_{j}} \leq z_{r+1}$, so that $R+1 \geq N-J$ with equality iff there is exactly one $x_{a_{j}}$ in each such interval. Thus $P$ has at least $N-2$ roots outside $(-1,1)$.

Since $P$ has degree $\leq N+1$, it has at most $N-1$ roots outside $(-1,1)$, so we must either have $Q+1=J$ or $R+1=N-J$. If (without loss of generality) $Q+1=J$ then $-1 / x_{a_{1}}<y_{1}$, so that $P\left(-1 / x_{a_{1}}\right)>0$ (by (5) again) and there must be a root of $P$ between $-1 / x_{a_{1}}\left(1 \leq-1 / x_{a_{1}}<y_{1}\right)$ and the last maximum of $F_{\Omega}$ in $(-1,1)$. This now forces $R+1=N-J$ also, hence $P\left(-1 / x_{a_{N}}\right)>0$ and there must be a root of $P$ between the first maximum of $F_{\Omega}$ in $(-1,1)$ and $-1 / x_{a_{N}}$ $\left(z_{R}<-1 / x_{a_{N}} \leq-1\right)$, contradicting $\operatorname{deg} P \leq N+1$.

Because of this result, in the rest of this section we shall mainly be interested in the influence of negative scalar curvature factors in the base metrics. In the presence of such factors, the existence of extremal Kähler metrics is nontrivial, as was already observed in [46] for ruled surfaces. By Theorem 2 such a metric exists in a given admissible class $\Omega$ iff the extremal polynomial $F_{\Omega}$ is positive on $(-1,1)$. However, the integrals $\alpha_{i}$ and $\beta_{j}$ involved in the above construction of $F_{\Omega}$ are hard to compute in general (see Appendix $\mathrm{B}$ ). For the next examples, we therefore adopt a different approach to compute $F_{\Omega}$. Instead of solving (4)-(5) and integrating, we solve first the boundary conditions.

It is easy to see that (6) together with (5) for $a=0$ and $a=\infty$ (if there are blow-downs) are solved by any $F$ of the form

$$
F(z)=\left(1-z^{2}\right)\left(p_{\mathrm{c}}(z)+(1+z)^{d_{0}+1}(1-z)^{d_{\infty}+1} q(z)\right)
$$


for some polynomial $q(z)$. Conversely any polynomial solution is of this form, and to obtain the extremal polynomial $F_{\Omega}$, the degree of $q$ must be $\leq\left(\sum_{a \in \mathcal{A}} d_{a}\right)-1$. Now it remains to compute $F^{\prime \prime}(z)$, to solve (4) -(5) for $a \in \mathcal{A}$ (so that $F=F_{\Omega}$ ), and to check positivity. For a given projective bundle and admissible Kähler class this leads to equations on the coefficients of $q$. The Futaki invariant will be zero (and the metric will be CSC) iff $q$ has degree $\leq\left(\sum_{a \in \mathcal{A}} d_{a}\right)-2$.

In general, the algebraic equations on $q$ are hopelessly complicated. However, when $S$ has real dimension $\leq 4$, they are tractable.

3.2. Extremal Kähler metrics over a Riemann surface. We consider first extremal Kähler metrics on projective bundles over a Riemann surface, generalizing the study of projective line bundles in [46].

Let $\Sigma$ be a compact Riemann surface with CSC metric $\left( \pm g_{\Sigma}, \pm \omega_{\Sigma}\right)$ and let $M=$ $P\left(E_{0} \oplus E_{\infty}\right) \rightarrow \Sigma$, where $E_{0}, E_{\infty}$ are projectively-flat hermitian vector bundles with ranks $d_{0}+1>0, d_{\infty}+1>0$, and $\bar{c}_{1}\left(E_{\infty}\right)-\bar{c}_{1}\left(E_{0}\right)=\left[\omega_{\Sigma} / 2 \pi\right]$. Let $\pm 2 s$ be the scalar curvature of $\pm g_{\Sigma}$ and $\Omega$ be an admissible Kähler class on $M$ defined by $0<|x|<1$. The metric

$$
g=(z+1) g_{0}+(z+1 / x) g_{\Sigma}+(z-1) g_{\infty}+\frac{p_{\mathrm{c}}(z)}{F(z)} d z^{2}+\frac{F(z)}{p_{\mathrm{c}}(z)} \theta^{2}
$$

is an extremal metric in the given class iff $F=F_{\Omega}$. From w3.1 we know that

$$
F_{\Omega}(z)=(1+z)^{d_{0}+1}(1-z)^{d_{\infty}+1}\left((1+x z)+c\left(1-z^{2}\right)\right)
$$

where $q(z)=c$ is a constant uniquely determined by the equation

$$
F_{\Omega}^{\prime \prime}(-1 / x)=2 s x(1-1 / x)^{d_{0}}(1+1 / x)^{d_{\infty}}
$$

(this holds whether or not $d_{0}, d_{\infty}$ are zero). We solve this to obtain

$$
c(s, x)=-\frac{2 x^{2}\left(2+d_{0}(1+x)+d_{\infty}(1-x)-s x\right)}{\left(2+d_{0}(1+x)+d_{\infty}(1-x)\right)\left(4+d_{0}(1+x)+d_{\infty}(1-x)\right)+\left(4+d_{0}+d_{\infty}\right)\left(1-x^{2}\right)} .
$$

Since $s x$ has same sign as $S c a l_{\Sigma}$, it can be positive only when $\Sigma=\mathbb{C} P^{1}$, in which case $E_{0}=\mathcal{L}_{0} \otimes \mathbb{C}^{d_{0}+1}$ and $E_{\infty}=\mathcal{L}_{\infty} \otimes \mathbb{C}^{d_{\infty}+1}$ for some line bundles $\mathcal{L}_{0}, \mathcal{L}_{\infty}$. It then follows that $\omega_{\Sigma}$ is integral, and thus $s=p / q$ where $p \leq 2$ and $q$ is an integer of same sign as $x \in(-1,1) \backslash\{0\}$ (see Remark 1); then we have that $s x<2$, so that $c<0$. Therefore $\mathfrak{F}_{\Omega}(K)$, which is a nonzero multiple of $c$, doesn't vanish for any admissible Kähler class. Since $b_{2}(\Sigma)=1$, every Kähler class on $M$ is admissible, so we get an immediate nonexistence result.

Theorem 5. Let $E_{0}, E_{\infty}$ be projectively-flat hermitian vector bundles over a Riemann surface $\Sigma$. Then there are no CSC Kähler metrics on $M=P\left(E_{0} \oplus E_{\infty}\right)$ unless $\bar{c}_{1}\left(E_{0}\right)=\bar{c}_{1}\left(E_{\infty}\right)$ (i.e., $E_{0} \oplus E_{\infty}$ is polystable).

This partially extends the converse in Corollary 1 to the case $\mathbf{g}>1$. Compared to Theorem 2, we note that here $\bar{c}_{1}\left(E_{\infty}\right)-\bar{c}_{1}\left(E_{0}\right)$ can never be strictly indefinite. On the other hand, by Theorem 2 we have an extremal Kähler metric for sufficiently small $\Omega$. Indeed it is easy to see that $|c|$ is small when $|x|$ is small, and hence $(1+x z)+c\left(1-z^{2}\right)$ is positive on $(-1,1)$. We also know from Proposition 11 that if $\Sigma$ has genus 0 or 1, then every admissible Kähler class contains an extremal Kähler metric. Let us now see what happens when $\mathbf{g}>1$, i.e., when $s x<0$.

Since $c<0$, the quadratic $Q(z)=(1+x z)+c\left(1-z^{2}\right)$ is concave. It is clearly positive at $z= \pm 1$, so it is positive on $(-1,1)$ unless its minimum is in $(-1,1)$ and it is nonpositive there. The minimum value $1+c+x^{2} / 4 c$ occurs at $z=x / 2 c$ and

$$
c(s, 1)=-\frac{2\left(1+d_{0}\right)-s}{2\left(1+d_{0}\right)\left(2+d_{0}\right)}, \quad c(s,-1)=-\frac{2\left(1+d_{\infty}\right)+s}{2\left(1+d_{\infty}\right)\left(2+d_{\infty}\right)} .
$$


It follows that if $s<-d_{0}\left(d_{0}+1\right)$, then $c(s, 1)<-\frac{1}{2}$ and hence for $0<x<1$ sufficiently close to 1 , we have $c(s, x)<-\frac{1}{2}$ and the minimum of $Q(z)$ is in $(-1,0)$ and nonpositive. Similarly, if $s>d_{\infty}\left(d_{\infty}+1\right)$ then for $-1<x<0$ sufficiently close to -1 , we have $c(s, x)<-\frac{1}{2}$ and the minimum of $Q(z)$ is in $(0,1)$ and nonpositive.

Hence if $\Sigma$ has genus $\mathbf{g}>1$ and $s<-d_{0}\left(d_{0}+1\right)$ or $s>d_{\infty}\left(d_{\infty}+1\right)$ then not every admissible Kähler class contains an admissible extremal metric.

Conversely if $s \geq-d_{0}\left(d_{0}+1\right)$ then it is easy to check that $c(s, x) \geq-x / 2$ for all $0<x<1$, so the minimum of $Q(z)$ is not in $(-1,1)$ for any such $x$, whereas if $s \leq d_{\infty}\left(d_{\infty}+1\right), c(s, x) \geq x / 2$ for all $-1<x<0$ and again the minimum of $Q(z)$ is not in $(-1,1)$ for any such $x$.

Theorem 6. Let $E_{0}, E_{\infty}$ be projectively-flat hermitian vector bundles of ranks $d_{0}+1, d_{\infty}+1$ over a compact Riemann surface $\Sigma$ of genus $\mathbf{g}$, and suppose $\bar{c}_{1}\left(E_{\infty}\right)-$ $\bar{c}_{1}\left(E_{0}\right)=\left[\omega_{\Sigma} / 2 \pi\right]$ for a Kähler form $\pm \omega_{\Sigma}$ of constant curvature. Then there exist admissible extremal Kähler metrics on $P\left(E_{0} \oplus E_{\infty}\right) \rightarrow \Sigma$. Such metrics exist in every Kähler class if $\mathbf{g}=0$ or 1 . For $\mathbf{g}>1$, put $\rho_{\Sigma}=s \omega_{\Sigma}$. Then such metrics exist in every Kähler class if and only if $-d_{0}\left(d_{0}+1\right) \leq s \leq d_{\infty}\left(d_{\infty}+1\right)$, otherwise such metrics exist for $|x|$ sufficiently small (depending on $s$ ).

Remark 7. In absence of blow-downs, we recover the examples of [46] on (complex) pseudo-Hirzebruch surfaces $P(\mathcal{O} \oplus \mathcal{L}) \rightarrow \Sigma$, where there are Kähler classes which do not contain an extremal Kähler metric (if $\Sigma$ has genus $\mathbf{g}>1$ ). Our result extends these examples to higher rank projective bundles. However, in the presence of blow-downs, there do exist projective bundles for which there is an extremal Kähler metric in every Kähler class, even with $\mathbf{g}>1$.

3.3. Nonexistence of CSC Kähler metrics over a Hodge 4-manifold. We now obtain a similar nonexistence result to Theorem [5 when $\operatorname{dim} S=4$.

Theorem 7. Let $\left(S, \pm g_{S}, \pm \omega_{S}\right)$ be a CSC Hodge 4-manifold and let $E_{0}, E_{\infty}$ be projectively-flat hermitian vector bundles of ranks $d_{0}+1, d_{\infty}+1$ over $S$ with $\bar{c}_{1}\left(E_{\infty}\right)-\bar{c}_{1}\left(E_{0}\right)=\left[\omega_{S} / 2 \pi\right]$. Then there are no CSC Kähler metrics in the admissible Kähler classes on $M=P\left(E_{0} \oplus E_{\infty}\right) \rightarrow S$.

Proof. We will prove that $\mathfrak{F}_{\Omega}(K)$ is nonzero for any admissible Kähler class $\Omega$ by showing that the leading coefficient of $F_{\Omega}$ cannot vanish. Following the discussion in 3.1 we see that for given $d_{0}, d_{\infty} \geq 0$ and $0<|x|<1$ we have

$$
F_{\Omega}(z)=(1+z)^{d_{0}+1}(1-z)^{d_{\infty}+1}\left((1+x z)^{2}+(c z+e)\left(1-z^{2}\right)\right)
$$

with $c$ and $e$ being constants uniquely determined by the conditions

$$
F_{\Omega}^{\prime \prime}(-1 / x)=0 \quad \text { and }\left.\quad \frac{F_{\Omega}^{\prime \prime}(z)}{1+x z}\right|_{z=-1 / x}=4 s x(1-1 / x)^{d_{0}}(1+1 / x)^{d_{\infty}} .
$$

The leading coefficient of $F_{\Omega}$ vanishes iff $c=0$. As before $c$ (and $e$ ) are determined by $s$ and $x$. In particular, $c(s, x)=n(s, x) / d(s, x)$, where

$$
\begin{aligned}
d(s, x) & =\left(1+d_{\infty}\right)\left(2+d_{\infty}\right)^{2}\left(3+d_{\infty}\right)(1-x)^{4} \\
& +4\left(2+d_{0}\right)\left(1+d_{\infty}\right)\left(2+d_{\infty}\right)\left(3+d_{\infty}\right)(1-x)^{3}(1+x) \\
& +6\left(2+d_{0}\right)\left(2+d_{\infty}\right)\left(4+d_{0}+d_{\infty}+\left(1+d_{0}\right)\left(1+d_{\infty}\right)\right)(1-x)^{2}(1+x)^{2} \\
& +4\left(1+d_{0}\right)\left(2+d_{0}\right)\left(3+d_{0}\right)\left(2+d_{\infty}\right)(1-x)(1+x)^{3} \\
& +\left(1+d_{0}\right)\left(2+d_{0}\right)^{2}\left(3+d_{0}\right)(1+x)^{4}
\end{aligned}
$$


(which is manifestly positive for $|x|<1$ ) and

$$
\begin{aligned}
-n(s, x) / 2 x^{3} & =4\left(6-3 s x+s x^{3}\right) \\
& +d_{0}(1+x)((5-x)(1+x)+(7-x)(3-s x)) \\
& +d_{\infty}(1-x)((5+x)(1-x)+(7+x)(3-s x)) \\
& +(9-s x)\left(d_{0}(1+x)+d_{\infty}(1-x)\right)^{2}+\left(d_{0}(1+x)+d_{\infty}(1-x)\right)^{3} .
\end{aligned}
$$

Since $s=p / q$ where $p \leq 3$ (see Remark 1) and $q$ is an integer of same sign as $x \in(-1,1) \backslash\{0\}$, we have that $s x<3$ and a moment's thought then gives that $n(s, x)$, and therefore $c(s, x)$, is never zero.

3.4. CSC Kähler metrics over a product of two Riemann surfaces. As counterpoint to the nonexistence results of $\$ \$ 3.2$ 3.3. we now explore explicitly the existence of CSC Kähler metrics, given by Theorem [2, in the simplest case when the base is a global product of two Riemann surfaces and there are no blow-downs.

Let $\Sigma_{a}(a=1,2)$ be compact Riemann surface with CSC metrics $\left( \pm g_{a}, \pm \omega_{a}\right)$ and let $M$ be $P(\mathcal{O} \oplus \mathcal{L}) \rightarrow \Sigma_{1} \times \Sigma_{2}$ where $\mathcal{L}=\mathcal{L}_{1} \otimes \mathcal{L}_{2}$ and $\mathcal{L}_{a}$ are pullbacks of line bundles on $\Sigma_{a}$ with $c_{1}\left(\mathcal{L}_{a}\right)=\left[\omega_{a} / 2 \pi\right]$. Let $\pm 2 s_{a}$ be the scalar curvature of $\pm g_{a}$ and $-1 / x_{a}$ be the constant roots defining an admissible Kähler class with $x_{1} \neq x_{2}$ (the case $x_{1}=x_{2}$ was considered in 3.3. where we established nonexistence of CSC metrics). We thus have $p_{\mathrm{c}}(z)=\left(1+x_{1} z\right)\left(1+x_{2} z\right)$ and the metric becomes

$$
g=\frac{1+x_{1} z}{x_{1}} g_{1}+\frac{1+x_{2} z}{x_{2}} g_{2}+\frac{p_{\mathrm{c}}(z)}{F(z)} d z^{2}+\frac{F(z)}{p_{\mathrm{c}}(z)} \theta^{2} .
$$

According to 3.1 to obtain a CSC metric, $F(z)$ must be the extremal polynomial

$$
F_{\Omega}(z)=\left(1-z^{2}\right)\left(\left(1+x_{1} z\right)\left(1+x_{2} z\right)+c x_{1} x_{2}\left(1-z^{2}\right)\right)
$$

where $c$ is a constant such that the following relations are satisfied

$$
F_{\Omega}^{\prime \prime}\left(-1 / x_{1}\right)=2 s_{1}\left(x_{1}-x_{2}\right), \quad F_{\Omega}^{\prime \prime}\left(-1 / x_{2}\right)=2 s_{2}\left(x_{2}-x_{1}\right) .
$$

Writing $2(1-c)=s$ (which is $\frac{1}{6} S c a l_{g}$ and not to be confused with the $s$ in the previous two paragraphs), these relations hold iff

$$
\begin{aligned}
& x_{1}\left(s_{1}\left(x_{1}-x_{2}\right)-2+(1-s) x_{1} x_{2}\right)+3(s-1) x_{2}=0 \\
& x_{2}\left(s_{2}\left(x_{2}-x_{1}\right)-2+(1-s) x_{1} x_{2}\right)+3(s-1) x_{1}=0,
\end{aligned}
$$

and these are precisely the conditions on an admissible Kähler class $\Omega$ (parameterized by $\left(x_{1}, x_{2}\right)$ with $0<\left|x_{a}\right|<1$ ) coming from the vanishing of $\mathfrak{F}_{\Omega}(K)$ (see Proposition 8). Eliminating $s=2(1-c)$, we obtain (using $x_{1} \neq x_{2}$ )

$$
x_{1}\left(6+s_{1} x_{1}\left(x_{2}^{2}-3\right)\right)+x_{2}\left(6+s_{2} x_{2}\left(x_{1}^{2}-3\right)\right)=0 .
$$

The normalized scalar curvatures $s_{a}$ are subject to the integrality conditions $s_{a}=2\left(1-\mathbf{g}_{a}\right) / q_{a}$ for $q_{a}$ a nonzero integer with the same sign as $x_{a}$, where $\mathbf{g}_{a}$ is the genus of $\Sigma_{a}$. In particular $s_{a} x_{a}<2$. This and (19) imply that $x_{1} x_{2}<0$; we thus get a nonexistence result in the case $x_{1} x_{2}>0$.

Theorem 8. Let $\left(\Sigma_{a}, \omega_{a}\right)(a=1,2)$ be compact CSC Riemann surfaces and $\mathcal{L}$ be a holomorphic vector bundle over $\Sigma_{1} \times \Sigma_{2}$ with $c_{1}(\mathcal{L})=\left[\left(\omega_{1}+\omega_{2}\right) / 2 \pi\right]$ (so that $c_{1}(\mathcal{L})$ is positive definite). Then there are no admissible Kähler classes on $M=P(\mathcal{O} \oplus \mathcal{L}) \cong P\left(\mathcal{O} \oplus \mathcal{L}^{-1}\right)$ containing a CSC Kähler metric. 
Remark 8. Note that we do not need to assume that the base is a global product of compact Riemann surfaces for the nonexistence result in the above theorem. It is sufficient to have a compact base $S$ that is a local product of Riemann surfaces with CSC and $s_{a} x_{a}<2$, which is always satisfied, since $S c a l_{ \pm g_{a}} \leq 4$ by the integrality of the pull-back of $\pm \omega_{a}$ to the universal cover of $S$.

In contrast to this result, we have the following observation.

Lemma 7. Let $\Omega$ be an admissible Kähler class, corresponding to a solution $\left(x_{1}, x_{2}\right)$ of (17)-(18) with $s \geq 0$. Then $\Omega$ admits an admissible CSC Kähler metric with scalar curvature $6 s$.

Proof. If (19) holds, the extremal polynomial $F_{\Omega}$ of an admissible Kähler class gives rise to a globally defined CSC Kähler metric iff $F_{\Omega}>0$ on $(-1,1)$. Let $Q(z)=F_{\Omega}(z) /\left(1-z^{2}\right)$, and observe that the coefficient of $z^{2}$ in this quadratic is $\frac{1}{2} s x_{1} x_{2}$. Since $Q( \pm 1)>0, Q$ will be positive on $[-1,1]$ if it is convex, i.e., if $s x_{1} x_{2}<0$. If $s=0, Q(z)$ is linear and positive on $[-1,1]$. Since $x_{1} x_{2}<0, F_{\Omega}$ is positive on $(-1,1)$ whenever we have solutions of (17)-18) with $s \geq 0$.

We now obtain some explicit solutions of (17)-(18). If we take $x_{2}$ (17) $-x_{1}$ (18) and $x_{1}(17)-x_{2}$ (18), we obtain, for $x_{1} \neq x_{2}$ :

$$
\begin{aligned}
\left(s_{1}+s_{2}\right) x_{1} x_{2} & =3(s-1)\left(x_{1}+x_{2}\right) \\
2\left(x_{1}+x_{2}\right) & =s_{1} x_{1}^{2}+s_{2} x_{2}^{2}+(1-s) x_{1} x_{2}\left(x_{1}+x_{2}\right) .
\end{aligned}
$$

These are equivalent to (17)-(18) for $x_{1}^{2} \neq x_{2}^{2}$. As $x_{1} \neq x_{2}, x_{1}^{2}=x_{2}^{2}$ iff $x_{1}+x_{2}=0$ and then $s_{1}+s_{2}=0$. The following lemma deals with this case.

Lemma 8. If $s_{1}+s_{2}=0$, then either $x_{1}+x_{2}=0$ and $s=\left(1-x_{1}^{2}+2 s_{1} x_{1}\right) /\left(3-x_{1}^{2}\right)$, or, without loss, $x_{1}=x_{2}+1, s=1$, and $s_{1}=2=-s_{2}$. Conversely, these give solutions of (17)-(18).

Proof. Clearly $s_{1}+s_{2}=0$ iff $s=1$ or $x_{1}+x_{2}=0$. The formula for $s$ in the latter case is immediate from (17). Now if $s=1$, then without loss of generality $s_{1}=-s_{2}$ is nonnegative and we must have either $x_{1}+x_{2}=0$, or $s_{1}>0$ and $x_{1}=x_{2}+2 / s_{1}$. Since $0<\left|x_{a}\right|<1$, this forces $x_{1}$ to be positive, hence $s_{1} \leq 2$, so in fact we must have $s_{1}=2$ and $x_{1}=x_{2}+1$.

In order to apply Lemma[7, we suppose in the first case above that $x_{1} s_{1} \geq 0$ : then $s>0$ since $1-x_{1}^{2}>0$ for $\left|x_{1}\right|<1$. Thus in both cases $s_{a} x_{a} \geq 0$ for $a=1,2$ and we obtain CSC Kähler metrics on projective line bundles over $T^{2} \times T^{2}, T^{2} \times \mathbb{C} P^{1}$ and $\mathbb{C} P^{1} \times \mathbb{C} P^{1}$. In particular any Kähler class on $P(\mathcal{O} \oplus \mathcal{O}(q,-q)) \rightarrow \mathbb{C} P^{1} \times \mathbb{C} P^{1}$ is admissible, so the above Lemmas and Proposition 11yield the following conclusions.

Theorem 9. On $P(\mathcal{O} \oplus \mathcal{O}(q,-q)) \rightarrow \mathbb{C} P^{1} \times \mathbb{C} P^{1}(q \geq 1)$, any Kähler class (parameterized, up to scale, by $0<x_{1}<1$ and $\left.-1<x_{2}<0\right)$ contains a unique admissible extremal Kähler metric. For $q>1$ this metric is CSC if and only if $x_{1}+x_{2}=0$, while for $q=1$ it is CSC if and only if $x_{1}+x_{2}=0$ or $x_{1}=x_{2}+1$.

When $q=1$, the two 1-parameter families of CSC Kähler classes of this theorem intersect at $x_{1}=1 / 2, x_{2}=-1 / 2$. In fact, the CSC metric in this Kähler class is the Koiso-Sakane Kähler-Einstein metric [29].

We end our study of CSC Kähler metrics on $P(\mathcal{O} \oplus \mathcal{L}) \rightarrow \Sigma_{1} \times \Sigma_{2}$, by considering the case of zero scalar curvature metrics, which we do not obtain automatically from Theorem 2. If $s=0$ then equation (17) defines $x_{2}$ as a function of $x_{1}$

$$
x_{2}=f_{1}\left(x_{1}\right)=x_{1} \frac{2-s_{1} x_{1}}{x_{1}^{2}-s_{1} x_{1}-3},
$$


whereas (18) defines $x_{1}$ as a function of $x_{2}$

$$
x_{1}=f_{2}\left(x_{2}\right)=x_{2} \frac{2-s_{2} x_{2}}{x_{2}^{2}-s_{2} x_{2}-3} .
$$

Note that $f_{1}(0)=f_{2}(0)=0$ and the gradients $d x_{2} / d x_{1}$ of the two graphs at $x_{1}=x_{2}=0$ are both negative. By comparing the size of the gradients one sees that for $x_{1}$ small and positive the graph of $f_{1}$ is above the graph of $f_{2}$. Note also that the denominator appearing in $f_{a}\left(x_{a}\right)$ is negative at $x_{a}=0$.

Assume that $s_{1} \leq 0$. If $f_{1}$ has no asymptotes for $0<x_{1}<1$ then $f_{1}(1) \leq-1$. Otherwise, for the asymptote $x_{1}=v$ closest to $x_{1}=0$ we have $\lim _{x_{1} \rightarrow v^{-}}=-\infty$. Assume moreover that $0<s_{2}$. If $f_{2}$ has no asymptotes for $-1<x_{2}<0$ then $f_{2}(-1)>1$. Otherwise, for the asymptote $x_{2}=v$ closest to $x_{2}=0$ we have $\lim _{x_{2} \rightarrow v^{+}}=+\infty$. By continuity the graphs of $f_{1}$ and $f_{2}$ intersect in the open square $(0,1) \times(0,-1)$ and (17)-(18) is solved for some $0<x_{1}<1$ and $-1<x_{2}<0$.

Theorem 10. Let $\left(\Sigma_{a}, \pm \omega_{a}\right)(a=1,2)$ be compact Riemann surfaces with genus $\mathrm{g}_{a}$ and canonical bundles $\mathcal{K}_{a}$, and suppose that the Kähler forms $\pm \omega_{a}$ are integral with constant curvature. Let $\mathcal{L}_{a}$ be line bundles on $\Sigma_{a}$ with $c_{1}\left(\mathcal{L}_{a}\right)=\left[\omega_{a} / 2 \pi\right]$ and, if $\mathbf{g}_{a} \neq 1$, let $\mathcal{L}_{a}$ be $\mathcal{K}_{a}^{q_{a} / 2\left(\mathbf{g}_{a}-1\right)}$ tensored by a flat line bundle, for an integer $q_{a}$.

Then, there is an admissible scalar-flat Kähler metric on $P\left(\mathcal{O} \oplus \mathcal{L}_{1} \otimes \mathcal{L}_{2}\right) \rightarrow$ $\Sigma_{1} \times \Sigma_{2}$ in the following cases:

- $\Sigma_{1}=T^{2}$ and $\mathcal{L}_{1}$ is ample, $\Sigma_{2}$ has genus $\mathbf{g}_{2}>1$ and $q_{2}<0$;

- $\Sigma_{1}$ and $\Sigma_{2}$ both have genus $\mathbf{g}_{a}>1, q_{1}>0$, and $q_{2}<0$.

\section{K-Stability AND ADMissible EXTREMAL KÄHLER METRICS}

4.1. Introduction to stability. For a Hodge manifold $(M, \Omega)$, the existence of a CSC Kähler metric in $\Omega$ is conjectured to be equivalent to a notion of stability 10 , 13, 14, 32, 39, 45, 47, for the polarized projective variety $(M, L)$, where $L$ is a line bundle on $M$ with $c_{1}(L)=\Omega / 2 \pi$. This conjecture is drawn from a detailed formal picture which makes clear an analogy with the well-established relation between the polystability of vector bundles and the existence of Einstein-Hermitian connections.

At present the most promising candidate for the conjectured stability criterion is 'K-polystability', in the form given by Donaldson [14, following Tian [45: a polarized projective variety $(M, L)$ is K-polystable if any 'test configuration' for $(M, L)$ has nonpositive Futaki invariant with equality iff the test configuration is a product. We shall explain this definition shortly. We also discuss an idea of Ross and Thomas 40, 41, who focus on test configurations arising as 'deformations to the normal cone' of subschemes of $(M, L)$, leading to a notion of 'slope' K-polystability analogous to the slope polystability of vector bundles. We explore this analogy further in 4.2

(Note that some authors use the term K-stable rather than K-polystable, but the latter term agrees better with pre-existing notions of stability.)

4.1.1. Finite dimensional motivation. Let $(X, \mathcal{L}, \Omega)$ be a polarized Kähler manifold with a hermitian metric on $\mathcal{L}$ with curvature $-i \Omega$ (thus $c_{1}(\mathcal{L})=\Omega / 2 \pi$ ). Suppose a compact connected group $G$ acts holomorphically on $X$ with momentum map $\mu: X \rightarrow \mathfrak{g}^{*}$ (i.e., $d\langle\mu, \xi\rangle=-\Omega\left(K_{\xi}, \cdot\right)$, where $K_{\xi}$ is the vector field on $X$ corresponding to $\xi \in \mathfrak{g}$, the Lie algebra of $G)$. There is a lift of the action to $\mathcal{L}$ generated by $\tilde{K}_{\xi}+\langle\mu, \xi\rangle K$ for each $\xi \in \mathfrak{g}$, where $\langle\mu, \xi\rangle$ is pulled back to $\mathcal{L}, \tilde{K}_{\xi}$ is the horizontal lift, and $K$ generates the standard $U(1)$ action on $\mathcal{L}$. The action of $\mathfrak{g}$ on $X$ and $\mathcal{L}$ extends to an action of the complexification $\mathfrak{g}^{c}$ and we assume this integrates to an action of a complex Lie group $G^{c}$. 
By a well-known result of Kempf-Ness and Kirwan, for any $x \in X$, there is a $g \in G^{c}$ such that $\mu(g \cdot x)=0$ iff for any nonzero lift $\tilde{x}$ of $x$ to $\mathcal{L}^{*}$, the orbit $G^{c} \cdot \tilde{x}=0$ is closed. Such points $x$ are said to be polystable. If $X^{p s}$ denotes the set of polystable points in $X$, we then have an equality between $X^{p s} / G^{c}$, the polystable quotient of $X$ by $G^{c}$, and the symplectic quotient $X / / G=\mu^{-1}(0) / G$.

$G^{c} \cdot \tilde{x}$ is closed iff $\alpha\left(\mathbb{C}^{\times}\right) \cdot \tilde{x}$ is closed for any one parameter subgroup $\alpha$ : $\mathbb{C}^{\times} \hookrightarrow$ $G^{c}$. This leads to the Hilbert-Mumford criterion for polystability: $x$ is said to be semistable if for any one parameter subgroup $\alpha: \mathbb{C}^{\times} \hookrightarrow G^{c}$, the linear action of $\mathbb{C}^{\times}$ on $\mathcal{L}_{x_{0}}^{*}$ has nonpositive weight $w_{x_{0}}(\alpha) \leq 0$, where $x_{0}=\lim _{\lambda \rightarrow 0} \alpha(\lambda) \cdot x$ is the limit point; $x$ is then polystable if it is semistable and $w_{x_{0}}(\alpha)=0$ only when $x_{0}=x$; finally $x$ is stable if it is polystable and has zero dimensional isotropy subgroup.

4.1.2. The infinite dimensional analogue. We apply the finite dimensional picture above formally to an infinite dimensional setting in which $X$ is the space of compatible complex structures on a compact symplectic manifold $(M, \omega)$ with $H^{1}(M)=0$. The space $X$ has a natural Kähler metric with respect to which the group $G$ of symplectomorphisms of $M$ acts holomorphically with a momentum map $\mu: X \rightarrow$ $C_{0}^{\infty}(M, \mathbb{R})$ given by the scalar curvature of the corresponding Kähler metric on $M$, modified by a constant in order to lie in $\mathfrak{g}^{*} \cong \mathfrak{g}=C_{0}^{\infty}(M, \mathbb{R})$, the functions with total integral zero, which is the Lie algebra of the symplectomorphism group equipped with the $L_{2}$-inner product. A quick way to see this is to observe that the Mabuchi K-energy (see 2.3 ) of $M$ is a Kähler potential for the metric on $X$ : the gradient on $X$ of the Mabuchi K-energy is the scalar curvature [21.

There is no group whose Lie algebra is the complexification $\mathfrak{g}^{c}$, but one can still consider the foliation of $X$ given by the vector fields induced by $\mathfrak{g}^{c}$. The complex structures in a given leaf are all biholomorphic by a diffeomorphism in the connected component of the identity, and pulling back the symplectic form $\omega$ by these biholomorphisms, we may identify the leaf with the set of all Kähler metrics in a fixed Kähler class, compatible with a fixed complex structure on $M$. Hence there should be a CSC metric in a given Kähler class iff the momentum map $\mu$ vanishes on the corresponding leaf iff the leaf is stable in a suitable sense.

To make precise this infinite dimensional analogue, we formalize what is meant by the orbit of a 1-parameter subgroup in terms of 'test configurations' and give a Hilbert-Mumford formulation of stability in terms of the weight of limit points.

4.1.3. Test configurations. Let $(M, \Omega)$ be a Hodge manifold, viewed as a polarized projective variety with respect to a line bundle $L$ with $c_{1}(L)=\Omega / 2 \pi$.

Definition 2. 14 A test configuration for $(M, L)$ is a polarized scheme $(X, \mathcal{E})$ over $\mathbb{C}$ with a $\mathbb{C}^{\times}$action $\alpha$ and a flat proper $\mathbb{C}^{\times}$-equivariant morphism $p: X \rightarrow \mathbb{C}$ (where $\mathbb{C}^{\times}$acts on $\mathbb{C}$ by scalar multiplication) such that the fibre $\left(X_{t}=p^{-1}(t),\left.\mathcal{E}\right|_{X_{t}}\right)$ is isomorphic to $(M, L)$ for some (hence all) $t \neq 0$.

$\left(X_{0},\left.\mathcal{E}\right|_{X_{0}}\right)$ is called the central fibre. Since $0 \in \mathbb{C}$ is fixed by the action, $\left(X_{0},\left.\mathcal{E}\right|_{X_{0}}\right)$ inherits a $\mathbb{C}^{\times}$action, also denoted by $\alpha$.

A test configuration is said to be a product configuration if $X=M \times \mathbb{C}$ and $\alpha$ is given by a $\mathbb{C}^{\times}$action on $M$ (and scalar multiplication on $\mathbb{C}$ ).

Since relevant properties of test configurations are unchanged if we replace $\mathcal{E}$ by $\mathcal{E}^{r}$ for a positive integer $r$, we can let $\mathcal{E}$ be a $\mathbb{Q}$-line bundle in the definition above (i.e., $\mathcal{E}$ denotes a 'formal root' of a line bundle $\mathcal{E}^{r}$ for some positive integer $r$ ).

A particularly important class of test configurations are those associated to a subscheme of $(M, L)$, as studied by Ross and Thomas [40, 41]. We shall state it 
here for complex submanifolds of $(M, L)$, but the same definition actually makes sense for subschemes.

Definition 3 (Deformation to the normal cone). For a polarized complex manifold $(M, L)$, the normal cone of a complex submanifold $Z$ is $\hat{M} \cup_{E} P$, where $\hat{M}$ is the blow-up of $M$ along $Z$ with exceptional divisor $E=P\left(\nu_{Z}\right), P=P\left(\mathcal{O} \oplus \nu_{Z}\right)$ and $\nu_{Z}$ is the normal bundle to $Z$ in $M$. This is a singular projective variety (for example the normal cone of a point $p \in \mathbb{C} P^{1}$ is $\mathbb{C} P^{1} \cup_{p} \mathbb{C} P^{1}$, which is a line-pair in $\mathbb{C} P^{2}$ ).

The normal cone is the central fibre of the family $p: X \rightarrow \mathbb{C}$ obtained by blowing up $M \times \mathbb{C}$ along $Z \times\{0\}$ (where $p$ is the projection of the blow-down to $\mathbb{C}$ ) called the deformation to the normal cone of $Z$ in $M$. We equip this with the polarization $\mathcal{E}_{c}=\pi^{*} L \otimes \mathcal{O}(-c P)$, where $\mathcal{O}(P)$ is the line bundle associated to the exceptional divisor $P, \pi: X \rightarrow M$ is the projection of the blow-down to $M$, and $c$ is a positive rational number such that $\mathcal{E}_{c}$ is an ample $\mathbb{Q}$-line bundle. This last condition gives an upper bound $\varepsilon$ on $c$, called the Seshadri constant of $Z$ with respect to $L$.

We let $\alpha$ be the $\mathbb{C}^{\times}$action coming from the trivial action on $M$ and multiplication on $\mathbb{C}$. This clearly defines an action on $X$ with a lift to $\mathcal{E}_{c}$. Hence the deformation to the normal cone determines a family of test configurations, parameterized by $c \in(0, \varepsilon) \cap \mathbb{Q}$.

4.1.4. The Futaki invariant and K-stability. K-stability is defined using a HilbertMumford criterion, i.e., in terms of a 'weight' associated to each test configuration. This weight is given by the Futaki invariant of the central fibre; however, since the latter is typically a singular projective variety, we need an algebraic geometric definition of the Futaki invariant. Such a definition has been given by Donaldson [14].

Let $V$ be a scheme of dimension $n$ over $\mathbb{C}$ polarized by an ample line bundle $L$ and suppose that $\alpha$ is a $\mathbb{C}^{\times}$action on $V$ with a lift to $L$. Then $\alpha$ acts on the vector spaces $H_{k}=H^{0}\left(V, L^{k}\right), k \in \mathbb{Z}^{+}$. If $w_{k}(\alpha)$ denotes the weight of the highest exterior power of $H_{k}$ (that is, the trace $\operatorname{Tr} A_{k}$ of the infinitesimal generator $A_{k}$ of the action) and $d_{k}$ denotes the dimension of $H_{k}$ then $w_{k}(\alpha)$ and $d_{k}$ are given by polynomials in $k$ for sufficiently large $k$, of degrees at most $n+1$ and $n$ respectively. For sufficiently large $k$ the quotient $w_{k}(\alpha) /\left(k d_{k}\right)$ can be expanded into a power series with no positive powers. The Futaki invariant $\mathfrak{F}(\alpha)$ is the residue at $k=0$ of this quotient, that is, the coefficient of the $k^{-1}$ term in the resulting expansion. The Futaki invariant is independent of the choice of lift of $\alpha$ to $L$. (When $V$ is a manifold, this definition coincides with Futaki's original definition up to a normalization convention.)

Definition 4. The Futaki invariant of a test configuration is defined to be the Futaki invariant $\mathfrak{F}(\alpha)$ of the central fibre, where $\alpha$ denotes the induced $\mathbb{C}^{\times}$action.

A Hodge manifold $(M, L)$ is said to be K-polystable if the Futaki invariant of any test configuration is nonpositive, and equal to zero if and only if the test configuration is a product configuration.

For the test configurations $\left(X, \mathcal{E}_{c}\right)$ arising from a deformation to a normal cone, Ross and Thomas [40, 41] show that the Futaki invariants $\mathfrak{F}\left(\alpha_{c}\right)$ are rational in $c \in(0, \varepsilon) \cap \mathbb{Q}$, where $\varepsilon$ is the Seshadri constant, and so can be extended to $c \in(0, \varepsilon)$.

Definition 5. A Hodge manifold $(M, L)$ is said to be slope K-polystable if for the deformation to the normal cone of any nontrivial subscheme, the Futaki invariant $\mathfrak{F}\left(\alpha_{c}\right)$ of the corresponding family $\left(X, \mathcal{E}_{c}\right)$ of test configurations is negative on $(0, \varepsilon)$.

Actually, the definition in [40, 41] is more subtle, since it requires that $\mathfrak{F}\left(\alpha_{\varepsilon}\right)<0$ unless $\varepsilon$ is rational and the semi-ample configuration $\left(X, \mathcal{E}_{\varepsilon}\right)$ is the pullback by a contraction of a product configuration. We shall not need this refinement. 
4.2. Stable bundles and CSC Kähler metrics. We now relate our results concerning CSC Kähler metrics on projective bundles to stability theory for vector bundles. Recall that if $E \rightarrow S$ is a holomorphic vector bundle over a compact kählerian $2 d$-manifold $\left(S,\left[\omega_{h}\right]\right)$, the slope $\mu(E)$ is the number $\bar{c}_{1}(E) \cdot\left[\omega_{h}\right]^{d-1} ; E$ is called (slope) stable or semistable if $\mu(F)<\mu(E)$ or $\mu(F) \leq \mu(E)$ (respectively) for any proper coherent subsheaf $F \subset E$; it is polystable if it is a direct sum of stable vector bundles with the same slope; then, as is well-known, 'stable' $\Rightarrow$ 'polystable' $\Rightarrow$ 'semistable', and by the Hitchin-Kobayashi correspondence, $E$ admits an Einstein-Hermitian connection iff it is polystable.

There is a close analogy between K-stability for polarized Kähler manifolds and slope stability for vector bundles. In particular, one might hope to find a direct relation between the existence problem for CSC Kähler metrics on a geometrically ruled complex manifold $P(E)$ over $S$ and the stability of $E \rightarrow S$. Notable progress in understanding the relation between K-polystability of $P(E)$ and slope polystability of $E$ has been made by Ross-Thomas [40, 41, using their notion of slope K-polystability: indeed if $F$ is a coherent subsheaf of $E$, then $P(F)$ is a subscheme of $P(E)$ and deformation to the normal cone of $P(F)$ is a test configaration which 'destabilizes' $P(E)$ iff $\mu(F)>\mu(E)$ (see [40]).

Using the general theory of CSC Kähler metrics, the work of [40] shows that if $E$ is not semistable with respect to an integral Kähler class $\left[\omega_{h}\right]$ on $S$, then for all $k \gg 1$ the integral classes $2 \pi c_{1}\left(\mathcal{O}(1)_{E}\right)+k p^{*}\left[\omega_{h}\right]$ on $P(E) \stackrel{p}{\rightarrow} S$ do not contain CSC metrics. As a partial converse, Hong [27, Theorem A] shows that if $E$ is polystable and $\mathfrak{h}_{0}(M) \rightarrow \mathfrak{h}_{0}(S)$ is surjective, then there is a CSC metric in $2 \pi c_{1}\left(\mathcal{O}(1)_{E}\right)+k p^{*}\left[\omega_{h}\right]$, for each $k \gg 1$, iff the Futaki invariant $\mathfrak{F}_{\Omega}$ vanishes.

To put our results in this context, let $P(E) \rightarrow S$ be admissible so $E=E_{0} \oplus E_{\infty}$ for projectively-flat (and thus polystable) hermitian vector bundles $E_{0}$ and $E_{\infty}$ with $\bar{c}_{1}\left(E_{\infty}\right)-\bar{c}_{1}\left(E_{0}\right)=\sum_{a}\left[\omega_{a} / 2 \pi\right]$. Thus $E$ is determined up to tensor product by a line bundle and is polystable iff it is semistable iff $\mu\left(E_{0}\right)=\mu\left(E_{\infty}\right)$. With respect to a Kähler class $\left[\omega_{h}\right]=\left[\sum_{a} \omega_{a} / f_{a}\right]$ on $S$ (where $f_{a} x_{a}>0$ ), this condition reads

$$
0=\mu\left(E_{\infty}\right)-\mu\left(E_{0}\right)=\frac{(d-1) !}{2 \pi} \operatorname{Vol}\left(S, \prod_{a} \omega_{a} / f_{a}\right)\left(\sum_{a} d_{a} f_{a}\right),
$$

which can happen for suitable $f_{a}$ iff $\bar{c}_{1}\left(E_{\infty}\right)-\bar{c}_{1}\left(E_{0}\right)$ is strictly indefinite; this is exactly the condition of Theorem 2 that ensures the existence of CSC metrics in a sufficiently small admissible Kähler class $\Omega=\Xi+p^{*}\left[\sum_{a \in \mathcal{A}} \omega_{a} / x_{a}\right]$, subject only to the constraint that $\mathfrak{F}_{\Omega}(K)=0$. Note that $\Xi$ is equal to $4 \pi c_{1}\left(\mathcal{O}(1)_{E}\right)$ up to a basic term (depending on the choice of $E$ ) - this follows by integrating $\Xi^{d_{0}+d_{\infty}+1}$ over a fibre and using the expression for $I\left(d_{0}, d_{\infty}, 0\right)$ from Appendix B. Thus, admissible classes play a similar role to those considered by Ross-Thomas and Hong, and $k \gg 1$ corresponds to $\left|x_{a}\right|$ sufficiently small in our picture. (We recall that this means that the fibres are small compared to the base.) However, there is not a simple relation in general between those $\Omega$ containing a CSC metric and the $\left[\omega_{h}\right]$ with respect to which $E$ is polystable: the approach of Ross-Thomas and Hong suggests taking $f_{a}=x_{a} /\left(1+r_{a} x_{a}\right)$, for some $r_{a}$ depending only on $E$; then (20) agrees asymptotically with $\mathfrak{F}_{\Omega}(K)=0$ in the limit $x_{a} \rightarrow 0$, but the two conditions define distinct hypersurfaces in general.

Conversely, in the case $E_{0}$ and $E_{\infty}$ are line bundles over a product $S=\Sigma_{1} \times \Sigma_{2}$ of two Riemann surfaces, Theorem 8 shows that polystability of $E$ with respect to some Kähler class on $S$ (which is unique up to scale in this case) is also necessary for the existence of a CSC metric in an admissible Kähler class on $P(E)$. 
Consider now the case that the base $S$ is a Riemann surface $\Sigma$ of genus g; the stability of a holomorphic vector bundle is then independent of the choice of a Kähler class on $\Sigma$, and it is natural to speculate [40] that the notion of $\mathrm{K}$ polystability of the projective manifold $P(E)$ should be independent of the specific Kähler class, and to conjecture that $P(E)$ admits a CSC Kähler metric iff $E$ is polystable. At present (see [2]) this conjecture is confirmed when $E$ is of rank 2 (i.e., on geometrically ruled surfaces), when $\mathbf{g} \leq 1$ and $E$ is a direct sum of line bundles (cf. Corollary 1 - this always holds when $\mathbf{g}=0$ ), or when $E$ is indecomposable and $\mathrm{g} \geq 2$. Theorem 5 further confirms the conjecture in the case of decomposable bundles of the form $E=E_{0} \oplus E_{\infty}$ with $E_{0}$ and $E_{\infty}$ polystable.

4.3. Extremal Kähler metrics and relative K-polystability. In recent work, G. Székelyhidi [44] has extended the theory of K-polystability to cover extremal Kähler metrics, not just CSC Kähler metrics. We briefly explain his ideas here.

4.3.1. Motivation. Recall that extremal Kähler metrics are critical points for the $L_{2}$-norm of the scalar curvature for metrics in a fixed Kähler class on a complex manifold $(M, J)$ [7. If we identify the Kähler class with a leaf of the formal $G^{c}$ orbit described in 4.1.2, we are therefore looking for critical points of $\|\mu\|^{2}$, where $\mu: X \rightarrow C_{0}^{\infty}(M, \mathbb{R})$ and $X$ is the space of compatible complex structures on a compact symplectic manifold $(M, \omega)$ with $H^{1}(M)=0$.

We can adapt the finite dimensional model of 4.1 .1 to this problem by supposing that the Lie algebra $\mathfrak{g}$ is equipped with a $G$-invariant inner product $\langle$,$\rangle . Now,$ following Székelyhidi [44, we note that the weight $w_{x}$ of the linear action of the isotropy algebra $\mathfrak{g}_{x}$ on $\mathcal{L}_{x}^{*}$ is given by $w_{x}=\left\langle\beta_{x}, \cdot\right\rangle: \mathfrak{g}_{x} \rightarrow \mathbb{C}^{\times}$for some $\beta_{x} \in \mathfrak{g}_{x}$, which is the orthogonal projection of $\mu(x)$ onto $\mathfrak{g}_{x}$. We refer to $\beta_{x}$ (or rather the induced vector field on $X$ ) as the extremal vector field: for in the infinite dimensional setting it agrees with the extremal vector field of Futaki and Mabuchi (see 2.2).

Clearly $x$ is a critical point of $\|\mu\|^{2}$ iff $\beta_{x}$ is in $\mathfrak{g}_{x}$. Using this, Székelyhidi shows that $x$ is in the $G^{c}$ orbit of a critical point of $\|\mu\|^{2}$ if and only if it is polystable relative to the extremal vector field, i.e., for the action of the subgroup of $G^{c}$ whose Lie algebra is the subspace $\beta_{x}^{\perp}$ of the centralizer of $\beta_{x}$. The HilbertMumford criterion may then be modified as follows: the modified weight $w_{x_{0}}(\alpha)-$ $\left\langle\alpha, \beta_{x}\right\rangle w_{x_{0}}\left(\beta_{x}\right) /\left\langle\beta_{x}, \beta_{x}\right\rangle$ of the limit point $x_{0}$ should be nonpositive for any one parameter subgroup $\alpha$ of the centralizer of $\beta_{x}$, with equality if and only if $x_{0}=x$.

4.3.2. The inner product and modified Futaki invariant. Thus motivated, we return to the setting of 4.1 .4 and define a modified Futaki invariant of a polarized scheme $(V, L)$ (of dimension $n$ over $\mathbb{C}$ ) relative to a $\mathbb{C}^{\times}$action $\beta$. We first need to define an inner product between such actions.

Assume then that $V$ has two $\mathbb{C}^{\times}$actions $\alpha$ and $\beta$ with lifts to $L$ and infinitesimal generators $A_{k}$ and $B_{k}$ of the actions on $H_{k}$. Then for $k$ sufficiently large, $\operatorname{Tr}\left(A_{k} B_{k}\right)$ is a polynomial of degree at most $n+2$. The inner product $\langle\alpha, \beta\rangle$ is defined to be the coefficient of $k^{n+2}$ of the expansion of $\operatorname{Tr}\left(A_{k} B_{k}\right)-w_{k}(\alpha) w_{k}(\beta) / d_{k}$ for large $k$, which is independent of the lifts of $\alpha$ and $\beta$ to $L$ : indeed it depends only on the trace-free parts of $A_{k}$ and $B_{k}$. (When $V$ is a manifold, this inner product coincides with Futaki-Mabuchi bilinear form [18] up to a normalization convention.)

We define the modified Futaki invariant 44 ] $\mathfrak{F}_{\beta}(\alpha)$ of $\alpha$ relative to $\beta$ (assuming the action $\beta$ is nontrivial) to be

$$
\mathfrak{F}_{\beta}(\alpha)=\mathfrak{F}(\alpha)-\frac{\langle\alpha, \beta\rangle}{\langle\beta, \beta\rangle} \mathfrak{F}(\beta) .
$$


4.3.3. Relative K-stability. Let $(M, \Omega, L)$ be as in 4.1 .3 and suppose it has a nontrivial $\mathbb{C}^{\times}$action $\beta$.

Definition 6. 44 A test configuration $(X, \mathcal{E})$ for $(M, L)$ is compatible with $\beta$ if there is a $\mathbb{C}^{\times}$action, also denoted by $\beta$, on $(X, \mathcal{E})$ preserving $p: X \rightarrow \mathbb{C}$ and inducing the trivial action on $\mathbb{C}$, such that $\beta$ restricted to $\left(X_{t},\left.\mathcal{E}\right|_{X_{t}}\right)$ coincides with the original action for $t \neq 0$ under the isomorphism with $(M, L)$.

In this case we have an induced action on the central fibre $X_{0}$, also called $\beta$, and the modified Futaki invariant of the test configuration is defined to be $\mathfrak{F}_{\beta}(\alpha)$.

A polarized Hodge manifold $(M, L)$ with nontrivial $\mathbb{C}^{\times}$action $\beta$ is $\mathrm{K}$-polystable relative to $\beta$ if the modified Futaki invariant of any test configuration $(X, \mathcal{E})$ compatible with $\beta$ is nonpositive, and equal to zero if and only if $(X, \mathcal{E})$ is a product.

If $(M, L)$ has a $\mathbb{C}^{\times}$action $\beta$ which preserves a subscheme $Z$, the test configurations $\left(X, \mathcal{E}_{c}\right)$ arising from the deformation to the normal cone are compatible with $\beta$. As in [40, 41, $\mathfrak{F}_{\beta}\left(\alpha_{c}\right)$ is rational in $c \in(0, \varepsilon) \cap \mathbb{Q}$ and so extends to $c \in(0, \varepsilon)$. Thus, cf. 4.1.4 we have a notion of slope K-polystability relative to $\beta$.

Definition 7. A polarized Hodge manifold $(M, L)$ with nontrivial $\mathbb{C}^{\times}$action $\beta$ is said to be slope $K$-polystable relative to $\beta$ if for the deformation to the normal cone of any nontrivial subsheme preserved by $\beta$, the modified Futaki invariant $\mathfrak{F}_{\beta}\left(\alpha_{c}\right)$ of the corresponding family $\left(X, \mathcal{E}_{c}\right)$ of test configurations is negative for $c \in(0, \varepsilon)$.

As with the definition of (absolute) slope K-polystability, strictly speaking, we should also require $\mathfrak{F}_{\beta}\left(\alpha_{\varepsilon}\right)<0$ unless $\varepsilon$ is rational and $\left(X, \mathcal{E}_{\varepsilon}\right)$ is the pullback by a contraction of a product configuration.

Nakagawa shows [37] that the (Futaki-Mabuchi) extremal vector field associated to a Hodge Kähler manifold $(M, \Omega)$ with a maximal compact connected subgroup $G$ of $H_{0}(M)$ has closed orbits, and therefore defines an effective $\mathbb{C}^{\times}$action which we will refer to as the extremal $\mathbb{C}^{\times}$action of $(M, \Omega, G)$. Then, the motivation of 4.3 .1 suggests the following conjecture 44.

Conjecture 1. Let $(M, \Omega, L)$ be a polarized Hodge manifold and $G$ a maximal compact connected subgroup of $H_{0}(M)$. Then there is a $G$-invariant extremal Kähler metric in $\Omega=2 \pi c_{1}(L)$ if and only if $(M, L)$ is K-polystable relative to the extremal $\mathbb{C}^{\times}$action of $(M, \Omega, G)$.

As a motivating example, Székelyhidi considers the deformation to the normal cone of a the infinity section in a (higher genus) polarized geometrically ruled surfaces $P(\mathcal{O} \oplus \mathcal{L}) \rightarrow \Sigma$. He finds that relative K-polystability implies existence of extremal Kähler metrics of the type constructed in [46, which are precisely the admissible metrics on these bundles. In the next section we generalize this idea to arbitrary admissible bundles. However, in doing so, we find that unless $\operatorname{dim} S \leq 4$, we need to replace 'K-polystable' by 'slope K-polystable' in the above conjecture.

4.4. Relative K-polystability of admissible projective bundles. We now consider the deformation to the normal cone $\left(X, \mathcal{E}_{c}, \alpha\right)$ of the infinity section $e_{\infty}=$ $z^{-1}(-1)=P\left(0 \oplus E_{\infty}\right)$ for an admissible projective bundle $M=P\left(E_{0} \oplus E_{\infty}\right) \rightarrow S$ (with $\operatorname{dim} S=2 d$ ), polarized by a line bundle $L$ with $\Omega=2 \pi c_{1}(L)$ admissible.

We therefore choose the admissible Kähler class $\Omega=\Xi+\sum_{a}\left[\omega_{a}\right] / x_{a}$ to be integral (where $0<\left|x_{a}\right| \leq 1$ with equality iff $a \in\{0, \infty\}$ ). The Seshadri constant of this polarization is 2 , so we take $c \in(0,2) \cap \mathbb{Q}$. Since the $\mathbb{C}^{\times}$action $\beta$ induced by the vector field $K$ preserves $Z, X$ is compatible. We will use the letters $\alpha, \beta$ to denote also the corresponding actions on the (polarized) central fibre $\left(X_{0}, L_{0}\right)$ and on the vector space $H^{0}\left(X_{0}, L_{0}^{k}\right)$, where $L_{0}=\left.\mathcal{E}_{c}\right|_{X_{0}}$. 
Let us calculate the modified Futaki invariant of this configuration. For this we first note that if $\mathcal{I}_{\infty} \subset \mathcal{O}_{M}$ is the ideal sheaf of holomorphic functions vanishing on $e_{\infty}$, then for any $p \geq 0, \mathcal{I}_{\infty}^{p} / \mathcal{I}_{\infty}^{p+1}$ is supported on $e_{\infty}$, and its restriction is $S^{p} \nu_{\infty}^{*}$, where $\nu_{\infty}$ is the normal bundle to $e_{\infty}$ in $M$.

Therefore, for $k$ sufficiently large, we have, as in [40, 44 ]

$$
H^{0}\left(X_{0}, L_{0}^{k}\right)=\bigoplus_{i=0}^{(2-c) k} H^{0}\left(e_{\infty},\left.L\right|_{e_{\infty}} ^{k} \otimes S^{2 k-i} \nu_{\infty}^{*}\right) \oplus \bigoplus_{j=1}^{c k} H^{0}\left(e_{\infty},\left.L\right|_{e_{\infty}} ^{k} \otimes S^{c k-j} \nu_{\infty}^{*}\right),
$$

where $\alpha$ acts on the first direct sum with weight 0 and on the components of the second direct sum with weight $-j$. We can choose the lift of $\beta$ to $L$ so that the weight of the induced action on $H^{0}\left(e_{\infty},\left.L\right|_{e_{\infty}} ^{k} \otimes S^{u k+v} \nu_{\infty}^{*}\right)$ is $(u-1) k+v$.

Now $S^{p} \nu_{\infty}^{*}$ is the direct image $q_{*} \mathcal{O}(p)_{\nu_{\infty}}$, where $\mathcal{O}(-1)_{\nu_{\infty}}$ is the (fibrewise) tautological bundle of $q: \hat{e}_{\infty}=P\left(\nu_{\infty}\right) \rightarrow e_{\infty}$. Also $\hat{e}_{\infty}$ may be identified with $\hat{S}$ via the obvious inclusion $i$ of $\hat{S}$ into $\hat{M}=P(\mathcal{O} \oplus \hat{\mathcal{L}})$ as the infinity section, and then $i^{*} \mathcal{O}(1)_{\nu_{\infty}}=\hat{\mathcal{L}}$. For convenience, we now drop the hats, so that we have

$$
\begin{aligned}
H^{0}\left(X_{0}, L_{0}^{k}\right) & =\bigoplus_{i=0}^{(1-z) k} H^{0}\left(S, i^{*} L^{k} \otimes \mathcal{L}^{2 k-i}\right) \oplus \bigoplus_{j=1}^{(1+z) k} H^{0}\left(S, i^{*} L^{k} \otimes \mathcal{L}^{(1+z) k-j}\right) \\
& =\bigoplus_{i=0}^{2 k} H^{0}\left(S, i^{*} L^{k} \otimes \mathcal{L}^{2 k-i}\right),
\end{aligned}
$$

where we have abused notation by writing $c-1=z$; a priori this has nothing to do with the momentum map that we also denote by $z$, but notice that it does take (rational) values in the same interval $(-1,1)$. To compute $d_{k}, \operatorname{Tr} A_{k}, \operatorname{Tr} B_{k}, \operatorname{Tr} A_{k} B_{k}$, $\operatorname{Tr} B_{k}^{2}$, and thereby $\mathfrak{F}_{\beta}(\alpha)$, we need only the dimensions of these vector spaces. We note that we only need to compute $d_{k}, \operatorname{Tr} A_{k}$ and $\operatorname{Tr} B_{k}$ to subleading order in $k$, whereas for $\operatorname{Tr} A_{k} B_{k}$ and $\operatorname{Tr} B_{k}^{2}$ the leading order term suffices. Consequently we will be dropping lower order terms without further comment. We also note that since the Futaki invariant is defined in terms of ratios, we can ignore any overall multiples. Now by the Riemann-Roch formula and the ampleness of $i^{*} L$ (in fact it is only semiample if $d_{\infty}>0$, but we can apply a limiting argument in this case, as in [40]), for sufficiently large $k$ we have that

$$
\begin{aligned}
h^{0}\left(S, i^{*} L^{k} \otimes \mathcal{L}^{u k+v}\right) & =\chi\left(S, i^{*} L^{k} \otimes \mathcal{L}^{u k+v}\right)=\left(\operatorname{ch}\left(i^{*} L^{k} \otimes \mathcal{L}^{u k+v}\right) \cdot \operatorname{Td}(S)\right)[S] \\
& =\left(c_{1}\left(i^{*} L^{k} \otimes \mathcal{L}^{u k+v}\right)+\frac{1}{2} c_{1}\left(\mathcal{K}_{S}^{-1}\right)\right)^{d}[S]+O\left(k^{d-2}\right) \\
& =\left(\sum_{a} \frac{k+\left((u-1) k+v+s_{a} / 2\right) x_{a}}{x_{a}}\left[\omega_{a} / 2 \pi\right]\right)^{d}[S]+O\left(k^{d-2}\right)
\end{aligned}
$$

since the Ricci form of $S_{a}$ may be written $s_{a} \omega_{a}+\rho_{a, 0}$ where $\rho_{a, 0} \wedge \omega_{a}^{d_{a}-1}=0$. After an overall multiplication by $(2 \pi)^{d} / d$ ! and $\prod_{a} x_{a}^{d_{a}} / \operatorname{Vol}\left(S_{a}, \omega_{a}\right)$, this is

$$
k^{d} p_{c}^{s}(u-1+v / k)+O\left(k^{d-2}\right),
$$

where $p_{c}^{s}(t)=\prod_{a}\left(1+x_{a}\left(t+s_{a} / 2 k\right)\right)^{d_{a}}$. In order to carry out the summations over $i$ and $j$ we use the trapezium rule, as in [40, Lemma 4.7].

Lemma 9. Let $f(x)$ be a polynomial and $b$ a rational number. Then for $\varepsilon \in\{0,1\}$ and for $k \in \mathbb{Z}^{+}$such that bk is a positive integer, we have

$$
\sum_{i=\varepsilon}^{b k} f(i / k)=k \int_{0}^{b} f(t) d t+\frac{1}{2}\left(f(b)+(-1)^{\varepsilon} f(0)\right)+O\left(k^{-1}\right) .
$$


The proof is easy (see e.g. [40]): by linearity we can assume $f(x)=x^{m}$ and then use $\sum_{i=1}^{N} i^{m}=N^{m+1} /(m+1)+N^{m} / 2+O\left(N^{m-1}\right.$ ) (which in turn is an easy induction on $N$ ). We then obtain (up to an overall multiple), that for any $r \geq 0$,

$$
\begin{aligned}
k^{-d-r} \operatorname{Tr} B_{k}^{r} & =k \int_{0}^{2 k}(1-t)^{r} p_{c}^{s}(1-t) d t+\frac{1}{2}\left(p_{c}(1)+(-1)^{r} p_{c}(-1)\right)+O(1 / k) \\
& =k \alpha_{r}+\frac{1}{2} \beta_{r}+O(1 / k),
\end{aligned}
$$

with $\alpha_{r}=\int_{-1}^{1} p_{c}(t) t^{r} d t$ and $\beta_{r}$ as in (77). Setting $r=0$ gives $d_{k}$. Similarly, using the explicit formula (12) for the extremal polynomial $F_{\Omega}(z)$ we obtain

$$
\begin{aligned}
k^{-d-1} \operatorname{Tr} A_{k}= & k \int_{0}^{1+z}-t p_{c}^{s}(z-t) d t-\frac{1}{2}(1+z) p_{c}(-1)+O(1 / k) \\
= & -k \int_{-1}^{z} p_{c}(t)(z-t) d t-\frac{1}{2} \int_{-1}^{z}\left(\sum_{a} \frac{d_{a} s_{a} x_{a}}{1+x_{a} t}\right) p_{c}(t)(z-t) d t \\
& -\frac{1}{2}(1+z) p_{c}(-1)+O(1 / k) \\
= & -k \int_{-1}^{z}(z-t) p_{c}(t) d t \\
& -\frac{1}{4} F_{\Omega}(z)+\frac{1}{4} \int_{-1}^{z}(A t+B) p_{c}(t)(z-t) d t+O(1 / k) \\
k^{-d-3} \operatorname{Tr} A_{k} B_{k}= & \int_{0}^{1+z}-t(z-t) p_{c}(z-t) d t+O(1 / k) \\
= & -\int_{-1}^{z} p_{c}(t) t(z-t) d t+O(1 / k)
\end{aligned}
$$

where $A$ and $B$ are the solutions of (9). Now we are ready to calculate $\langle\beta, \beta\rangle$, $\langle\alpha, \beta\rangle, \mathfrak{F}(\beta)$, and $\mathfrak{F}(\alpha)$. (We omit the dependence of $z$ for convenience.)

$$
\begin{aligned}
\langle\beta, \beta\rangle & =\frac{\alpha_{2} \alpha_{0}-\alpha_{1}^{2}}{\alpha_{0}} \\
\langle\alpha, \beta\rangle & =-\int_{-1}^{z} p_{c}(t) t(z-t) d t+\frac{\alpha_{1}}{\alpha_{0}} \int_{-1}^{z} p_{c}(t)(z-t) d t \\
\mathfrak{F}(\alpha) & =\operatorname{Res}_{k=0} \frac{\left(\operatorname{Tr} A_{k}\right)_{1}+\left(\operatorname{Tr} A_{k}\right)_{0} / k}{\alpha_{0}\left(1+\beta_{0} /\left(2 k \alpha_{0}\right)\right)}=\frac{\alpha_{0}\left(\operatorname{Tr} A_{k}\right)_{0}-\frac{1}{2} \beta_{0}\left(\operatorname{Tr} A_{k}\right)_{1}}{\alpha_{0}^{2}} \\
& =\frac{-\frac{1}{4} \alpha_{0} F_{\Omega}(z)+\frac{1}{4} \alpha_{0} \int_{-1}^{z}(A t+B) p_{c}(t)(z-t) d t+\frac{1}{2} \beta_{0} \int_{-1}^{z} p_{c}(t)(z-t) d t}{\alpha_{0}^{2}} \\
\mathfrak{F}(\beta) & =\operatorname{Res}_{k=0} \frac{\alpha_{1}+\beta_{1} / 2 k}{\alpha_{0}\left(1+\beta_{0} /\left(2 k \alpha_{0}\right)\right)}=\frac{\beta_{1} \alpha_{0}-\beta_{0} \alpha_{1}}{2 \alpha_{0}^{2}}
\end{aligned}
$$

where we have set $\left(\operatorname{Tr} A_{k}\right)_{0}=-\frac{1}{4} F_{\Omega}(z)+\frac{1}{4} \int_{-1}^{z}(A t+B) p_{c}(t)(z-t) d t$ and $\left(\operatorname{Tr} A_{k}\right)_{1}=$ $-k \int_{-1}^{z} p_{c}(t)(z-t) d t$.

Finally, we can calculate the modified Futaki invariant for our test configuration.

$$
\begin{aligned}
& \alpha_{0}^{2} \mathfrak{F}_{\beta}(\alpha)=\alpha_{0}^{2}(\mathfrak{F}(\alpha)-\langle\alpha, \beta\rangle \mathfrak{F}(\beta) /\langle\beta, \beta\rangle) \\
& =-\frac{1}{4} \alpha_{0} F_{\Omega}(z)+\frac{1}{4} \alpha_{0} \int_{-1}^{z}(A t+B) p_{c}(t)(z-t) d t+\frac{1}{2} \beta_{0} \int_{-1}^{z} p_{c}(t)(z-t) d t \\
& \quad+\frac{\alpha_{0}\left(\beta_{1} \alpha_{0}-\beta_{0} \alpha_{1}\right)}{2\left(\alpha_{2} \alpha_{0}-\alpha_{1}^{2}\right)}\left(\int_{-1}^{z} p_{c}(t) t(z-t) d t-\frac{\alpha_{1}}{\alpha_{0}} \int_{-1}^{z} p_{c}(t)(z-t) d t\right)
\end{aligned}
$$




$$
\begin{aligned}
= & -\frac{1}{4} \alpha_{0} F_{\Omega}(z)+\frac{1}{4} \alpha_{0} \int_{-1}^{z}(A t+B) p_{c}(t)(z-t) d t-\frac{1}{4} \alpha_{0} \int_{-1}^{z} A t p_{c}(t)(z-t) d t \\
& +\frac{\beta_{0}\left(\alpha_{2} \alpha_{0}-\alpha_{1}^{2}\right)-\alpha_{1}\left(\beta_{1} \alpha_{0}-\beta_{0} \alpha_{1}\right)}{2\left(\alpha_{2} \alpha_{0}-\alpha_{1}^{2}\right)} \int_{-1}^{z} p_{c}(t)(z-t) d t \\
= & -\frac{1}{4} \alpha_{0} F_{\Omega}(z)+\frac{1}{4} \alpha_{0} \int_{-1}^{z}(A t+B) p_{c}(t)(z-t) d t \\
& -\frac{1}{4} \alpha_{0} \int_{-1}^{z} A t p_{c}(t)(z-t) d t-\frac{1}{4} \alpha_{0} \int_{-1}^{z} B p_{c}(t)(z-t) d t \\
= & -\frac{1}{4} \alpha_{0} F_{\Omega}(z)
\end{aligned}
$$

which is a negative multiple of the extremal polynomial. It follows immediately that if $(M, L)$ is slope $\mathrm{K}$-polystable relative to $K=J \operatorname{grad}_{g} z$, then $F_{\Omega}$ is positive on $(-1,1)$ and $\Omega$ contains an admissible extremal metric by Theorem 2

If $(M, L)$ is slope $\mathrm{K}$-polystable in the absolute sense, then $\mathfrak{F}(\alpha)$ is negative on $(-1,1)$ and hence nonpositive at $z=1$. Evaluating the integrals in this case (and using $F_{\Omega}(1)=0$ ), we find that $A \geq 0$. Now if we swap the roles of the zero and infinity sections (by interchanging $E_{0}$ and $E_{\infty}$ ) then the analogous calculation shows that $A \leq 0$ (we get the same formulae with the change of variables $z \mapsto-z$ ). Thus $A=0$ and $\mathfrak{F}(\beta)=0$. (Intuitively, the reason we get $\mathfrak{F}(\beta)=0$ is that in these limits, deformation to the normal cone of the zero or infinity section is actually the pullback by a contraction of the product configuration associated to $\pm \beta$, cf. [40, 41.) Hence $(M, L)$ is slope $\mathrm{K}$-polystable relative to $\beta, F_{\Omega}$ is positive on $(-1,1)$, and the admissible extremal metric is CSC.

This proves Theorem [3. providing evidence for the reverse implication in Conjecture 1 (with relative K-stability replaced by relative slope K-stability) because in our setting, the extremal vector field is a nonzero multiple of $A K$. This calculation also shows that the forward implication in Conjecture 1 implies Corollary 2 without referring to K-energy or the results of Chen-Tian [10, 11, providing further indirect evidence. However, if we use relative K-stability instead of relative slope K-stability, we can only deduce that $F_{\Omega}$ is positive on $(-1,1) \cap \mathbb{Q}$ and hence nonnegative on $(-1,1)$. However, since $\Omega$ is integral, $F_{\Omega}$ has rational coefficients, and so when $\operatorname{dim} S\left(=\sum_{a \in \mathcal{A}} 2 d_{a}\right) \leq 4$ it follows that $F_{\Omega}$ is positive on $(-1,1)$ : indeed $F_{\Omega}(z)=(1+z)^{d_{0}+1}(1-z)^{d_{\infty}+1} Q(z)$ where $Q(z)$ is a quadratic or cubic with rational coefficients, and the repeated roots of such a polynomial must be rational.

On the other hand, the following examples show that positivity of the extremal polynomial on $(-1,1) \cap \mathbb{Q}$ is not sufficient for the existence of an extremal Kähler metric when $\operatorname{dim} S=6$.

Example 1. Let $S=\Sigma_{1} \times \Sigma_{2} \times \Sigma_{3}$ be a product of hyperbolic Riemann surfaces $\Sigma_{a}$ with integral Kähler classes $\left[ \pm \omega_{a}\right]$. Then for any admissible projective line bundle $M$ over $S$ and any admissible integral Kähler class $\Omega$ on $M$ with parameters $x_{a} \in \mathbb{Q}$, the extremal polynomial has the form

$$
F_{\Omega}(z)=\left(1-z^{2}\right)\left(p_{c}(z)+\left(1-z^{2}\right)\left(a_{0}+a_{1} z+a_{2} z^{2}\right)\right)
$$

where the $a_{j}$ are determined by the constant gaussian curvatures $\pm s_{a}$ of $\Sigma_{a}$ (via (44)(5)). However, since we are free to choose the genera and degrees of the line bundles defining $M$, the $s_{a}$ can be arbitrary rational numbers subject only to the constraint that $s_{a} x_{a}<0$ (so that the gaussian curvatures are negative). Hence we are free to choose the $a_{j}$ subject to this constraint. 
We claim that for any rational $r>0$ and $x_{1}>x_{2}>0>x_{3}$, we can choose the $a_{j}$ so that $F_{\Omega}(z)$ is a positive multiple of $\left(1-z^{2}\right)\left(z^{2}+r z-1\right)^{2}$ provided that

$$
x_{1} x_{2} x_{3}+x_{1}+x_{2}+x_{3}=0 .
$$

$F_{\Omega}$ then has a repeated root in $(0,1)$ and another in $(-\infty,-1)$ and for $r$ in an open subset of $\mathbb{Q}^{+}$, these roots are irrational. Obviously for any $1>x_{1}>x_{2}>0$ rational, (21) has a unique rational solution $x_{3}=-\left(x_{1}+x_{2}\right) /\left(1+x_{1} x_{2}\right)$ with $0>x_{3}>-1$, and it is elementary to verify our claim by equating coefficients. $F_{\Omega}^{\prime \prime}(z)$ is then negative for large $z$ and has at least two roots in $(-1,1)$ and none in $(1, \infty)$. We then check that for $r>8 / 5, F_{\Omega}^{\prime \prime}(-1)$ is negative and so the other two roots (which must be real, since $F_{\Omega}$ must have four inflection points) are in $(-\infty,-1)$. Hence we can choose $x_{1}, x_{2}$ so that $s_{1} x_{1}<0$ and $s_{2} x_{2}<0$, with $s_{a}$ defined by (4) -(5). It automatically follows that $s_{3} x_{3}<0$.

These data then define a countably infinite family (parameterized by $\left(x_{1}, x_{2}, r\right)$ in an open subset of $\mathbb{Q}^{3}$ ) of admissible projective line bundles over products of three Riemann surfaces together with admissible rational Kähler classes (which we can scale to be integral) such that $F_{\Omega}$ is positive on $(-1,1) \cap \mathbb{Q}$, but has an irrational repeated root in $(-1,1)$. By Theorem 2 these Kähler classes then do not contain an extremal Kähler metric.

\section{Appendix A. Relation to previous papers}

In this appendix we summarize the classification of compact Kähler $2 m$-manifolds $M$ with a hamiltonian 2-form of order $\ell$ given in [5. Theorem 2], and explain how Theorem 1 follows from this classification in the case $\ell=1$. We also give a nonexistence result for extremal Kähler metrics when $\ell=2$.

A.1. Summary of the classification. Let $(M, g, J, \omega)$ be a compact connected Kähler $2 m$-manifold with a hamiltonian 2 -form $\phi$ of order $\ell$. Let $p(t)$ be the momentum polynomial of $\phi$ and $K(t)=J \operatorname{grad}_{g} p(t)$ be the corresponding family of hamiltonian Killing vector fields. We summarize results from [4, 5] in italics.

The vector fields $\{K(t): t \in \mathbb{R}\}$ generate an effective isometric hamiltonian action of an $\ell$-torus $\mathbb{T}$ on $M$ and $p(t)$ has $m-\ell$ constant roots counted with multiplicity. This action is free on a connected dense open subset $M^{0}$ of $M$.

We let $S_{\Delta}$ be the stable quotient of $M$ by the induced action of the complexified torus $\mathbb{T}^{c}$ and denote by $\eta_{a}$, for $a$ in a finite set with $\leq m-\ell$ elements, the distinct constant roots of $p(t)$ and by $d_{a}$ their multiplicities.

$S_{\Delta}$ is covered by a product $\tilde{S}_{\Delta}=\prod_{a} S_{a}$ of Kähler $2 d_{a}$-manifolds $\left(S_{a}, \pm g_{a}, \pm \omega_{a}\right)$, and $M^{0} \rightarrow S_{\Delta}$ is a principal $\mathbb{T}^{c}$-bundle.

In [4, 5, we took $a \in\{1, \ldots N\}$, but here we shall adopt (in a moment) a different notation for the index set. We let $p_{\mathrm{c}}(t)=\prod_{a}\left(t-\eta_{a}\right)^{d_{a}}$ and write $p(t)=p_{\mathrm{c}}(t) p_{\mathrm{nc}}(t)$, where $p_{\text {nc }}(t)=\sum_{r=0}^{\ell}(-1)^{r} \sigma_{r} t^{\ell-r}$ and $\sigma_{0}=1$. The Killing vector fields $K_{r}:=$ $J \operatorname{grad}_{g} \sigma_{r}$, for $r=1, \ldots \ell$, are linearly independent on $M^{0}$.

The image $\Delta$ of the momentum map $\left(\sigma_{1}, \ldots \sigma_{\ell}\right)$ is a simplex in $\mathfrak{t}^{*} \cong \mathbb{R}^{\ell}$, whose interior (the image of $M^{0}$ ) is the image under the elementary symmetric functions of a domain $D=\prod_{j=1}^{\ell}\left(\beta_{j-1}, \beta_{j}\right)$, where $\beta_{0}<\beta_{1}<\cdots<\beta_{\ell}$. The roots of $p_{\mathrm{nc}}(t)$ define smooth, functionally independent, pairwise distinct functions $\xi_{j}(j=1, \ldots \ell)$ on $M^{0}$ which extend continuously to $M$ with image $\left[\beta_{j-1}, \beta_{j}\right]$. The codimension one faces of $\Delta$ may be labelled $F_{0}, \ldots F_{\ell}$ such that on $F_{j}$, either $\xi_{j}=\beta_{j}$ or $\xi_{j+1}=\beta_{j}$.

The local description of the metric on $M^{0}$ is as follows. 
There are 1 -forms $\theta_{1}, \ldots \theta_{\ell}$ on $M^{0}$ with $\theta_{r}\left(K_{s}\right)=\delta_{r s}$ and $d \theta_{r}=\sum_{a}(-1)^{r} \eta_{a}^{\ell-r} \omega_{a}$ and a function $\Theta$ of one variable satisfying

$$
\begin{gathered}
(-1)^{\ell-j} \Theta>0 \quad \text { on } \quad\left(\beta_{j-1}, \beta_{j}\right), \\
\Theta\left(\beta_{j}\right)=0, \quad \Theta^{\prime}\left(\beta_{j}\right)=-\prod_{k \neq j}\left(\beta_{j}-\beta_{k}\right),
\end{gathered}
$$

such that the Kähler structure on $M^{0}$ may be written

$$
\begin{aligned}
& g=\sum_{a} p_{\mathrm{nc}}\left(\eta_{a}\right) g_{a}+\sum_{j=1}^{\ell} \frac{\Delta_{j}}{\Theta\left(\xi_{j}\right)} d \xi_{j}^{2}+\sum_{j=1}^{\ell} \frac{\Theta\left(\xi_{j}\right)}{\Delta_{j}}\left(\sum_{r=1}^{\ell} \sigma_{r-1}\left(\hat{\xi}_{j}\right) \theta_{r}\right)^{2}, \\
& \omega=\sum_{a} p_{\mathrm{nc}}\left(\eta_{a}\right) \omega_{a}+\sum_{r=1}^{\ell} d \sigma_{r} \wedge \theta_{r},
\end{aligned}
$$

where $\sum_{a} p_{\mathrm{nc}}\left(\eta_{a}\right) g_{a}$ is the pullback of a local Kähler product metric on $\hat{S}, \Delta_{j}=$ $\prod_{k \neq j}\left(\xi_{j}-\xi_{k}\right)$, and $\sigma_{r}\left(\hat{\xi}_{j}\right)$ is the rth elementary symmetric function of $\xi_{1}, \ldots \xi_{\ell}$ with $\xi_{j}$ omitted. ( $\sigma_{r}$ itself is the rth elementary symmetric function of $\xi_{1}, \ldots \xi_{\ell}$.)

The global description of $M$ in [5, Theorem 2] was presented using the blow-up $\hat{M}$ of $M$ along the inverse image of the codimension one faces $F_{0}, \ldots F_{\ell}$ of $S_{\Delta}$. $\hat{M}$ is $\mathbb{T}^{c}$-equivariantly biholomorphic to the $\mathbb{C} P^{\ell}$-bundle $M^{0} \times_{\mathbb{T}^{c}} \mathbb{C} P^{\ell} \rightarrow S_{\Delta}$.

The blow-up is encoded by fibrations $S_{\Delta} \rightarrow S_{F_{j}}$ for each $F_{j}$ (see also [5. Proposition 6]): either $S_{\Delta}=S_{F_{j}}$, or the fibration is covered by the obvious projection $\tilde{S}_{\Delta} \rightarrow \prod_{b \neq a_{j}} S_{b}$ for some index $a_{j}$ such that $S_{a_{j}}$ is a complex projective space and $\left( \pm g_{a_{j}}, \pm \omega_{a_{j}}\right)$ has constant holomorphic sectional curvature $\pm \prod_{k \neq j}\left(\beta_{j}-\beta_{k}\right)$

We unify these cases here by introducing, if $S_{\Delta}=S_{F_{j}}$, an additional index $a_{j}$ with $d_{a_{j}}=0$ and $S_{a_{j}}=\mathbb{C} P^{0}$ (a point). We denote the new index set by $\hat{\mathcal{A}}$ and take $a \in \hat{\mathcal{A}}$ unless otherwise stated: the additional indices make no difference to the previous formulae. We still have $\tilde{S}_{\Delta}=\prod_{a} S_{a}$, and now for all $F_{j}, S_{\Delta} \rightarrow S_{F_{j}}$ is

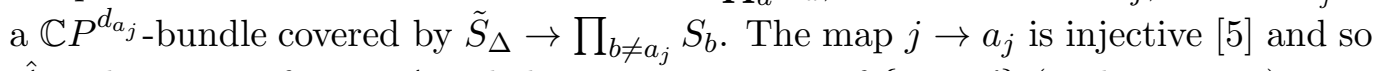
$\hat{\mathcal{A}}$ is the union of a set $\mathcal{A}$ and the injective image of $\{0, \ldots \ell\}$ (under $j \mapsto a_{j}$ ).

For $a \in \mathcal{A}$, either $\eta_{a}<\beta_{0}$ or $\eta_{a}>\beta_{\ell}$, according to the sign of $\left( \pm g_{a}, \pm \omega_{a}\right)$, whereas for $j=\{0, \ldots \ell\}, \eta_{a_{j}}=\beta_{j}$.

The formula (24) for the metric on $M^{0}$ leads to a description [5] of $\hat{M}$ as a projective bundle $P\left(\mathcal{L}_{0} \oplus \mathcal{L}_{1} \oplus \cdots \oplus \mathcal{L}_{\ell}\right) \rightarrow S_{\Delta}$ together with formulae for the Chern classes of $\mathcal{L}_{j}$ on the covering $\tilde{S}_{\Delta}$. To obtain instead a description of $M$, we need one further ingredient, which follows easily by considering the form of the covering transformations and the fact that $\tilde{S}_{\Delta} \rightarrow \prod_{b \neq a_{j}} S_{b}$ covers the fibration $S_{\Delta} \rightarrow S_{F_{j}}$.

Lemma 10. The projection $\tilde{S}_{\Delta} \rightarrow \tilde{S}:=\prod_{a \in \mathcal{A}} S_{a}$ descends to realize $S_{\Delta}$ as a fibre product of flat projective unitary $\mathbb{C} P^{d_{a_{j}}}$-bundles over a quotient $S$ of $\tilde{S}$.

An important class of flat projective unitary $\mathbb{C} P^{r}$-bundles on $S$ are those of the form $P(E)$, where $E$ is a rank $r+1$ projectively-flat hermitian vector bundle on $S$. If $S$ is simply connected, then any flat projective unitary $\mathbb{C} P^{r}$-bundle is trivial, hence of the form $P(E)$ with $E \cong \mathcal{E} \otimes \mathbb{C}^{r+1}$ for a holomorphic line bundle $\mathcal{E}$. In general the obstruction to the existence of $E$ is given by a torsion element of $H^{2}\left(S, \mathcal{O}^{*}\right)$ (cf. [16]). In particular, such an $E$ always exists if $S$ is a Riemann surface.

Let us suppose that $S_{\Delta}=P\left(E_{0}\right) \times_{S} P\left(E_{1}\right) \times_{S} \cdots \times_{S} P\left(E_{\ell}\right) \rightarrow S$, where each $E_{j} \rightarrow S$ is projectively-flat hermitian of rank $d_{j}+1$. We are free to choose the $E_{j}$ so that $\hat{M}=P\left(\mathcal{O}(-1)_{E_{0}} \oplus \mathcal{O}(-1)_{E_{1}} \oplus \cdots \oplus \mathcal{O}(-1)_{E_{\ell}}\right)$ where $\mathcal{O}(-1)_{E_{j}}$ is the 
(fibrewise) tautological line bundle over $P\left(E_{j}\right)$ (trivial over the other factors of $S_{\Delta}$ ). From the description of the blow-up in [5], we immediately deduce the following (in which we write $\left.\bar{c}_{1}(E)=c_{1}(E) / \operatorname{rank} E\right)$.

$M$ is $\mathbb{T}^{c}$-equivariantly biholomorphic to $P\left(E_{0} \oplus E_{1} \cdots \oplus E_{\ell}\right) \rightarrow S$ and for any $i \neq j$, $\bar{c}_{1}\left(E_{j}\right)-\bar{c}_{1}\left(E_{i}\right)=\frac{1}{2} \sum_{a}\left(\prod_{k \neq i}\left(\eta_{a}-\beta_{k}\right)-\prod_{k \neq j}\left(\eta_{a}-\beta_{k}\right)\right)\left[\omega_{a} / 2 \pi\right]$.

Derivation of Theorem [1. In order to derive Theorem 1 from the above, it suffices to rescale $g$ so that we can take $\beta_{0}=-1$ and $\beta_{1}=1$. Then we set $\eta_{a}=-1 / x_{a}$ and change the sign of $\omega_{a}$ for all $a$. We also write $\hat{S}$ for $S_{\Delta}$, and replace the index set $\{0,1\}$ by $\{0, \infty\}$ so that we can take $\hat{\mathcal{A}}=\{0, \infty\} \cup \mathcal{A}$ where $\mathcal{A}$ is a finite subset of $\mathbb{Z}^{+}$, but these changes are purely cosmetic.

A.2. A nonexistence result for order 2 extremal Kähler metrics. In this paper we study only hamiltonian 2-forms of order 1 . As a partial justification for this restriction, we now consider the lowest interesting dimension for the order 2 case, and show that any extremal metric on a compact Kähler 6-manifold compatible with a hamiltonian 2-form of order 2 is a Fubini-Study metric on $\mathbb{C} P^{3}$.

In this situation, the momentum polynomial has nonconstant roots $\xi_{1}$ and $\xi_{2}$ and one constant root $\eta$ so $\# \mathcal{A} \leq 1$ and $p(t)=(t-\eta)\left(t-\xi_{1}\right)\left(t-\xi_{2}\right)$. The stable quotient $\Sigma$ of $(M, J)$ by the complexified $\mathbb{T}^{c}$ action is a compact Riemann surface with Kähler structure $\left(g_{\Sigma}, \omega_{\Sigma}\right)$.

We can set $\beta_{0}=-1$ and $\beta_{2}=1$ and write $\beta_{1}=\beta$ (where $|\beta|<1$ ). If $\mathcal{A}$ is empty, $(M, J)$ is biholomorphic to $\mathbb{C} P^{3}$; otherwise $|\eta|>1$ and $(M, J)$ is $\mathbb{T}^{c}$-equivariantly biholomorphic to $M=P\left(\mathcal{L}_{0} \oplus \mathcal{L}_{1} \oplus \mathcal{L}_{2}\right) \rightarrow \Sigma$, where $\mathcal{L}_{j}$ are holomorphic line bundles on $\Sigma$ such that (without loss) $\mathcal{L}_{1}$ is trivial and

$$
\begin{aligned}
& c_{1}\left(\mathcal{L}_{0}\right)=\frac{1}{2}(\eta-1)(\beta+1)\left[\omega_{\Sigma} / 2 \pi\right], \\
& c_{1}\left(\mathcal{L}_{2}\right)=\frac{1}{2}(\eta+1)(\beta-1)\left[\omega_{\Sigma} / 2 \pi\right] .
\end{aligned}
$$

The Kähler metric on $M$ is determined by a function $\Theta(t)$ satisfying positivity and boundary conditions which imply that $\Theta(t)=F(t) /(t-\eta)$ where $F(t)=$ $H(t)((t-\eta)+H(t) Q(t))$ for some function $Q(t)$, and $H(t)=\left(1-t^{2}\right)(t-\beta)$.

If $g$ is extremal and the extremal vector field is tangent to the fibres of $M \rightarrow \Sigma$, then by [4, $F(t)$ is a polynomial of degree at most 5 and $g_{\Sigma}$ has scalar curvature $-F^{\prime \prime}(\eta)$. This forces $Q(t)=0$ and so the scalar curvature of $g_{\Sigma}$ is $2\left(3 \eta^{2}-2 \beta \eta-1\right)$ which is positive since $|\eta|>1$ and $|\beta|<1$. Hence $\Sigma=\mathbb{C} P^{1}$. Since $\frac{1}{4} \operatorname{Scal}_{g_{\Sigma}}\left[\omega_{\Sigma} / 2 \pi\right]$ is a primitive integral class, (25) implies that

$$
(\eta \mp 1)(\beta \pm 1)=q^{ \pm}\left(3 \eta^{2}-2 \beta \eta-1\right)
$$

for some nonzero integers $q^{ \pm}$. We remark that these formulae show that the relation between $q^{ \pm}$and $(\eta, \beta)$ is birational, in fact the restriction to $\mathbb{R}^{2}$ of a quadratic transformation of $\mathbb{C} P^{2}$. In any case, $\eta$ is constant on the lines through $\left(q^{+}, q^{-}\right)=$ $(1,1)$, and $\beta= \pm 1$ on the lines $q^{ \pm}=0$ and $2 q^{ \pm}-q^{\mp}=1$, the latter being the lines on which $\eta= \pm 1$. It follows straightforwardly that $|\eta|>1$ and $|\beta|<1$ iff $q^{+}>0$, $q^{-}<0$ and $2 q^{+}-q^{-}<1$ or vice-versa (swap plus and minus) - which is impossible as $\left|q_{ \pm}\right| \geq 1$. We therefore have the following nonexistence result.

Theorem 11. A compact extremal Kähler 6-manifold $(M, J, g, \omega)$ which admits a hamiltonian 2-form of order 2 with the extremal vector field tangent to the $\mathbb{T}^{c}$-orbits is isometric to $\mathbb{C} P^{3}$ with a Fubini-Study metric. 


\section{Appendix B. Proof of Lemma 6}

In this appendix we prove Lemma 6 by computing the asymptotics as $x_{a} \rightarrow 0$, for $a \in \mathcal{A}$, of the solution $(A, B)$ of the system (9), i.e., $A \alpha_{1}+B \alpha_{0}=-2 \beta_{0}$, $A \alpha_{2}+B \alpha_{1}=-2 \beta_{1}$, where $\alpha_{r}=\int_{-1}^{1} p_{\mathrm{c}}(t) t^{r} d t$ and $\beta_{r}$ are as in (77). In order to do this, we rewrite $\beta_{0}$ and $\beta_{1}$ as integrals using the obvious identities

$$
\begin{aligned}
& p_{\mathrm{c}}(1)+p_{\mathrm{c}}(-1)=\int_{-1}^{1} \frac{d}{d t}\left(t p_{\mathrm{c}}(t)\right) d t=\int_{-1}^{1}\left(1+\sum_{a} \frac{d_{a} x_{a} t}{1+x_{a} t}\right) p_{\mathrm{c}}(t) d t \\
& p_{\mathrm{c}}(1)-p_{\mathrm{c}}(-1)=\int_{-1}^{1} \frac{d}{d t}\left(t^{2} p_{\mathrm{c}}(t)\right) d t=\int_{-1}^{1}\left(2+\sum_{a} \frac{d_{a} x_{a} t}{1+x_{a} t}\right) p_{\mathrm{c}}(t) t d t
\end{aligned}
$$

to obtain

$$
\begin{aligned}
\beta_{0}=\int_{-1}^{1}(1+t)^{d_{0}}(1-t)^{d_{\infty}}\left(1+d_{0}+d_{\infty}+\frac{d_{0}^{2}}{1+t}+\frac{d_{\infty}^{2}}{1-t}+\sum_{a \in \mathcal{A}} \frac{d_{a} x_{a}\left(s_{a}+t\right)}{1+x_{a} t}\right) \\
\quad \times\left(\prod_{a \in \mathcal{A}}\left(1+x_{a} t\right)^{d_{a}}\right) d t \\
\beta_{1}=\int_{-1}^{1}(1+t)^{d_{0}}(1-t)^{d_{\infty}}\left(2+d_{0}+d_{\infty}+\frac{d_{0}^{2}}{1+t}+\frac{d_{\infty}^{2}}{1-t}+\sum_{a \in \mathcal{A}} \frac{d_{a} x_{a}\left(s_{a}+t\right)}{1+x_{a} t}\right) \\
\quad \times\left(\prod_{a \in \mathcal{A}}\left(1+x_{a} t\right)^{d_{a}}\right) t d t .
\end{aligned}
$$

The asymptotics of $\alpha_{0}, \alpha_{1}, \alpha_{2}, \beta_{0}$ and $\beta_{1}$ are given by integrals of the form

$$
I(m, n, k)=\int_{-1}^{1}(1+t)^{m}(1-t)^{n} t^{k} d t .
$$

Integrating by parts and using $2 I(m, n, k+1)=I(m+1, n, k)-I(m, n+1, k)$,

$$
\begin{aligned}
I(m, n, 0) & =\frac{2^{m+n+1} m ! n !}{(m+n+1) !}, \quad I(m, n, 1)=\frac{2^{m+n+1}(m-n) m ! n !}{(m+n+2) !}, \\
I(m, n, 2) & =\frac{2^{m+n+1}\left(m^{2}+n^{2}+m+n-2 m n+2\right) m ! n !}{(m+n+3) !} .
\end{aligned}
$$

These are rather complicated, so we manipulate the integrals using the identities

$$
\begin{aligned}
I(m-1, n, 0) m^{2}+I(m, n-1,0) n^{2}= & \frac{1}{2} I(m, n, 0)(m+n+1)(m+n) \\
I(m-1, n, 1) m^{2}+I(m, n-1,1) n^{2}= & \frac{1}{2} I(m, n, 1)(m+n-1)(m+n+2) \\
I(m-1, n, 2) m^{2}+I(m, n-1,2) n^{2}= & \frac{1}{2} I(m, n, 2)(m+n+3)(m+n) \\
& -I(m, n, 1)(m-n)
\end{aligned}
$$

and thus obtain, up to $O\left(x^{2}\right)$,

$$
\begin{aligned}
\alpha_{k} & =I\left(d_{0}, d_{\infty}, k\right)+I\left(d_{0}, d_{\infty}, k+1\right) \sum_{a \in \mathcal{A}} d_{a} x_{a} \\
\beta_{0} & =\frac{1}{2} I\left(d_{0}, d_{\infty}, 0\right)\left(1+d_{0}+d_{\infty}\right)\left(2+d_{0}+d_{\infty}\right)+I\left(d_{0}, d_{\infty}, 0\right) \sum_{a \in \mathcal{A}} d_{a} s_{a} x_{a} \\
& +\frac{1}{2} I\left(d_{0}, d_{\infty}, 1\right)\left(1+d_{0}+d_{\infty}\right)\left(2+d_{0}+d_{\infty}\right) \sum_{a \in \mathcal{A}} d_{a} x_{a}
\end{aligned}
$$




$$
\begin{aligned}
\beta_{1} & =\frac{1}{2} I\left(d_{0}, d_{\infty}, 1\right)\left(1+d_{0}+d_{\infty}\right)\left(2+d_{0}+d_{\infty}\right)+I\left(d_{0}, d_{\infty}, 1\right) \sum_{a \in \mathcal{A}} d_{a} s_{a} x_{a} \\
& +\left(\frac{1}{2} I\left(d_{0}, d_{\infty}, 2\right)\left(3+d_{0}+d_{\infty}\right)\left(2+d_{0}+d_{\infty}\right)-I\left(d_{0}, d_{\infty}, 1\right)\left(d_{0}-d_{\infty}\right)\right) \sum_{a \in \mathcal{A}} d_{a} x_{a} .
\end{aligned}
$$

Direct computation with these formulae and the identity $I(m, n, 1)(m+n+2)=$ $I(m, n, 0)(m-n)$ now shows that, up to $O\left(x^{2}\right)$,

$$
\begin{aligned}
& \frac{\alpha_{0} \beta_{1}-\alpha_{1} \beta_{0}}{\alpha_{0} \alpha_{2}-\alpha_{1}^{2}}=\left(2+d_{0}+d_{\infty}\right) \sum_{a \in \mathcal{A}} d_{a} x_{a} \\
& \frac{\alpha_{2} \beta_{0}-\alpha_{1} \beta_{1}}{\alpha_{0} \alpha_{2}-\alpha_{1}^{2}}=\frac{1}{2}\left(1+d_{0}+d_{\infty}\right)\left(2+d_{0}+d_{\infty}\right)+\sum_{a \in \mathcal{A}} d_{a} s_{a} x_{a}+\left(d_{\infty}-d_{0}\right) \sum_{a \in \mathcal{A}} d_{a} x_{a} .
\end{aligned}
$$

Multiplying by -2 completes the proof.

\section{REFERENCES}

[1] M. F. Atiyah, Convexity and commuting hamiltonians, Bull. London Math. Soc. 14 (1982), $1-15$.

[2] V. Apostolov and C. Tønnesen-Friedman, A remark on Kähler metrics of constant scalar curvature on ruled complex surfaces, Bull. London Math. Soc., to appear, arXiv:math.DG/0411271

[3] V. Apostolov, D. M. J. Calderbank and P. Gauduchon, The geometry of weakly self-dual Kähler surfaces, Compositio Math. 135 (2003), 279-322.

[4] V. Apostolov, D. M. J. Calderbank and P. Gauduchon, Hamiltonian 2-forms in Kähler geometry I General Theory, J. Diff. Geom., to appear, arXiv:math.DG/0202280

[5] V. Apostolov, D. M. J. Calderbank, P. Gauduchon and C. Tønnesen-Friedman, Hamiltonian 2-forms in Kähler geometry II Global Classification, J. Diff. Geom. 68 (2004), 277-345.

[6] S. Bando and T. Mabuchi, Uniqueness of Einstein-Kähler metrics modulo connected group actions, in 'Algebraic Geometry' (Sendai 1985), Adv. Stud. Pure Math. 10, North Holland, 1987, 11-40.

[7] E. Calabi, Extremal Kähler metrics, Seminar on Differential Geometry, Princeton Univ. Press (1982), 259-290.

[8] E. Calabi, Extremal Kähler metrics II, in: Differential Geometry and Complex Analysis (eds. I. Chavel and H.M. Farkas), Springer, Berlin, 1985.

[9] X. X. Chen, Space of Kähler metrics, J. Diff. Geom. 56 (2000), 189-234.

[10] X. X. Chen and G. Tian, Geometry of Kähler metrics and holomorphic foliation by discs, arXiv:math.DG/0409433

[11] X. X. Chen and G. Tian, Geometry of Kähler metrics and foliations by holomorphic discs, arXiv:math.DG/0507148

[12] J.-P. Demailly, $L^{2}$ vanishing theorems for positive line bundles and adjunction theory, in 'Transcendental methods in algebraic geometry' (Cetraro, 1994), Lecture Notes in Math. 1646, Springer, Berlin, 1996, 1-97.

[13] S. Donaldson, Scalar curvature and projective embeddings I, J. Diff. Geom. 59 (2001), 479-522.

[14] S. Donaldson, Scalar curvature and stability of toric varieties, J. Diff. Geom. 62 (2002), 289349 .

[15] S. Donaldson, Scalar curvature and projective embeddings II, arXiv:math.DG/0407534

[16] G. Elencwajg and M. S. Narasimhan, Projective bundles on a complex torus, J. Reine Angew. Math. 340 (1983), 1-5.

[17] A. Futaki, Kähler-Einstein metrics and Integral Invariants, Lecture Notes in Math. 1314, Springer, Berlin, 1988.

[18] A. Futaki and T. Mabuchi, Bilinear forms and extremal Kähler vector fields associated with Kähler classes, Math. Ann. 301 (1995), 199-210.

[19] A. Fujiki, Remarks on extremal Kähler metrics on ruled manifolds, Nagoya Math. J. 126 (1992), 89-101.

[20] T. Fujita, On polarized manifolds whose adjoint bundles are not semipositive, in 'Algebraic Geometry' (Sendai, 1985), 167-178, Adv. Stud. in Pure Math. 10, North Holland, 1987.

[21] P. Gauduchon, Calabi's extremal metrics: An elementary introduction, in preparation. 
[22] D. Guan, Existence of extremal metrics on compact almost homogeneous Kähler manifolds with two ends, Trans. Amer. Math. Soc. 347 (1995), 2255-2262.

[23] D. Guan, On modified Mabuchi functional and Mabuchi moduli space of Kähler metrics on toric bundles, Math. Res. Lett. 6 (1999), 547-555.

[24] V. Guillemin, Kähler structures on toric varieties, J. Diff. Geom. 40 (1994), 285-309.

[25] A. D. Hwang, On existence of Kähler metrics with constant scalar curvature, Osaka J. Math. 31 (1994), 561-595.

[26] A. D. Hwang and M. A. Singer, A momentum construction for circle-invariant Kahler metrics, Trans. Amer. Math. Soc. 354 (2002), 2285-2325.

[27] Y.-J. Hong, Gauge-fixing constant scalar curvature equations on ruled manifolds and the Futaki invariant, J. Diff. Geom. 60 (2002), 389-453.

[28] S. Kobayashi, Differential Geometry of Complex Vector Bundles, Publications of the Math. Soc. of Japan, Kan Memorial Lectures 5, Princeton Univ. Press, Princeton, Iwanami Shoten, Tokyo, 1987.

[29] N. Koiso and Y. Sakane, Nonhomogeneous Kähler-Einstein metrics on compact complex manifolds, in Curvature and Topology of Riemannian Manifolds (Kataka, 1985), Lecture Notes in Math. 1201, Springer, Berlin, 1986, 165-179.

[30] C. R. LeBrun and S. Simanca, On the Kähler classes of extremal Kähler metrics, in 'Geometry and Global Analysis', International Research Institute, Sendai, 1993, 255-271.

[31] A. Lichnerowicz, Sur les transformations analytiques des variétés kählériennes, C.R. Acad. Sci. Paris 244 (1957), 3011-3014.

[32] H. Luo, Geometric criterion for Gieseker-Mumford stability of polarized manifolds, J. Diff. Geom. 49 (1998), 577-599.

[33] T. Mabuchi, Some symplectic geometry on compact Kähler manifolds, Osaka J. Math. 24 (1987), 227-252.

[34] T. Mabuchi, An energy-theoretic approach to the Hitchin-Kobayashi correspondence for manifolds II, J. Diff. Geom., to appear.

[35] T. Mabuchi, Uniqueness of extremal Kähler metrics for an integral Kähler class, Int. J. Math. 15 (2004), 531-546.

[36] Y. Matsushima, Sur la structure du groupe d'homéomorphismes analytiques d'une certaine variété kählérienne, Nagoya Math. J. 11 (1957), 145-150.

[37] Y. Nakagawa, Bando-Calabi-Futaki characters of Kähler orbifolds, Math. Ann. 314 (1999), 369-380.

[38] M.S. Narasimhan and C.S. Seshadri, Stable and unitary vector bundles on a compact Riemann surface, Ann. Math. 82 (1965), 540-567.

[39] S. Paul and G. Tian, Algebraic and analytic K-stability, Int. Math. Res. Notices 48 (2004), 2555-2591.

[40] J. Ross and R. Thomas, An obstruction to the existence of constant scalar curvature Kähler metrics, arXiv:math.DG/0412518

[41] J. Ross and R. Thomas, A study of the Hilbert-Mumford criterion for the stability of projective varieties, arXiv:math.DG/0412519

[42] S. R. Simanca, A K-energy characterization of extremal Kähler metrics, Proc. Amer. Math. Soc. 128 (2000), 1531-1535.

[43] T. Suwa, Ruled surfaces of genus one, J. Math. Soc. Japan 2 (1969), 258-291.

[44] G. Székelyhidi, Extremal metrics and K-stability, arXiv:math.AG/0410401

[45] G. Tian, On Calabi's conjecture for complex surfaces with positive first Chern class, Invent. Math. 101 (1990), 101-172.

[46] C. Tønnesen-Friedman, Extremal Kähler metrics on minimal ruled surfaces, J. reine angew. Math. 502 (1998), 175-197.

[47] S. Zhang, Heights and reductions of semistable varieties, Compostio Math. 104 (1996), 77105. 
40 V. APOSTOLOV, D. CALDERBANK, P. GAUDUCHON, AND C. TØNNESEN-FRIEDMAN

Vestislav Apostolov, Département de Mathématiques, UQAM, C.P. 8888, Succ. Centre-ville, Montréal (Québec), H3C 3P8, Canada

E-mail address: apostolo@math.uqam.ca

David M. J. Calderbank, Department of Mathematics, University of York, HesLINGTON, YORK YO10 5DD, ENGLAND

E-mail address: dc511@york.ac.uk

Paul Gauduchon, Centre de Mathématiques, École Polytechnique, UMR 7640 du CNRS, 91128 Palaiseau, France

E-mail address: pg@math.polytechnique.fr

Christina W. Tønnesen-Friedman, Department of Mathematics, Union College, SCHENECTADY, NEW YORK 12308, USA

E-mail address: tonnesec@union.edu 